\title{
8 Relationen zwischen Konstruktionen und Frames
}

\author{
While most efforts in the FrameNet project have \\ focused on particular lexical items and their \\ frames, it stands to reason that also \\ constructions can evoke frames.
}

(Hilpert 2009: 38)

Nach der Entwicklung eines konstruktionssemantischen Modells in Kapitel 4, der Identifikation sieben semantischer Parameter von Konstruktionen in Kapitel 5 und der Analyse der Strukturparallelen zwischen Konstruktionen und Frames in Kapitel 6 haben die konstruktikographischen Generalisierungen in Kapitel 7 die Untersuchung der reflexiven Bewegungskonstruktion, der reflexiven Partikelverbkonstruktion und der reflexiven Weg-Konstruktion prinzipiell abgeschlossen. Die vorliegende Arbeit könnte an dieser Stelle also beendet sein, da alle Informationen, die für die drei Konstruktionseinträge dieser Konstruktionen relevant sind, ermittelt wurden. Damit jedoch sind längst nicht alle konstruktionssemantisch interessanten Fragen beantwortet. Eine dieser Fragen hätte eigentlich noch vor der Entfaltung des konstruktionssemantischen Modells in Kapitel 4 adressiert werden müssen. Warum wird für die drei untersuchten Konstruktionen gerade Motion als Konstruktions-Frame angesetzt? Die entsprechende Annahme stützt sich bislang lediglich auf vorgängige Analysen der englischen way-Konstruktion (etwa von Fillmore, Lee-Goldman \& Rhomieux 2012: 322), in denen er als KonstruktionsFrame postuliert wird (vgl. Unterabschnitt 2.3.2). Doch auch diese Analysen geben keine Auskunft darüber, wie sie zu der Entscheidung für Motion als KonstruktionsFrame kommen. ${ }^{1}$ Die Frage, welcher Konstruktions-Frame für eine Konstruktion angesetzt werden muss, ist, wie nebenbei auch Zeschel (2018: 69) betont, eine konstruktikographisch fundamentale.

Bisher habe ich die auch für diese Arbeit getroffene Entscheidung für Motion ebenso wenig hinterfragt wie die Selbstverständlichkeit, mit der bisher davon ausgegangen ist, dass in den Konstrukten der drei untersuchten Konstruktionen Motion als Konstruktions-Frame ,evoziert' wird, ebenso wie die im jeweiligen KE EREIGNIS instanziierten LE lexikalische Frames evozieren. Hier allerdings ist eine Differenzierung notwendig: Während Frames im lexikalischen Verständnis

1 Wie bereits in Unterabschnitt 2.3.2 erwähnt, wird für die way-Konstruktion bisweilen statt Motion auch Self_motion als Konstruktions-Frame angesetzt (etwa von Lee-Goldman \& Petruck 2018: 32-33). Ich komme darauf in Unterabschnitt 8.5.1 zurück. 
von FrameNet durch LE evoziert werden, also durch lexikalisch fixierte Elemente, lässt sich dies für syntaktische Konstruktionen nicht in jedem Fall behaupten.

Dass diese Aspekte konstruktikographische Relevanz haben, zeigt die Analysepraxis des Konstruktikons für das Brasilianische Portugiesisch. So argumentieren Torrent et al. (2014), dass die konstruktikographische Modellierung von Strukturparallelen zwischen Konstruktionen und Frames, etwa zwischen KE und FE (genauer: zwischen KtE und FE, vgl. Abschnitt 6.2) zwangsläufig zu der Annahme von Relationen zwischen Konstruktionen und Frames führen muss:

The computational implementation of such mappings [zwischen KE und FE, A.W.] [...] comprises the creation of a construction-to-frame relation in the database - the Evokes relation - and of constructional units (CUs) in the frames evoked by the constructions. (Torrent et al. 2014: 45)

Eine solche Evokationsrelation kann als eigenständige konstruktikographische Beschreibungseinheit angesehen werden und muss Eingang in eine entsprechende datenbanktechnische Modellierung erfahren (vgl. Laviola et al. 2017: 194-195; Matos et al. 2017: 223; Torrent et al. 2018b: 127). Über die Natur dieser Evokationsrelation, über die Frage also, wie man sich die Relation ${ }^{2}$ zwischen einer Konstruktion (genauer: ihrer Formseite) und ihrem Konstruktions-Frame vorzustellen hat und welche Mechanismen einen Konstruktions-Frame in Abgrenzung zu einem lexikalischen Frame ,evozieren', ist damit aber noch nichts ausgesagt. Ebenso wenig finden sich bisher methodologische Reflexionen darüber, wie der KonstruktionsFrame einer Konstruktion überhaupt zu ermitteln ist.

Um die beiden soeben skizzierten Fragenkomplexe soll es in diesem Kapitel gehen. Anknüpfend an das konstruktionssemantische Modell und die Analysen der Strukturparallelen zwischen Konstruktionen und Frames möchte ich in Abschnitt 8.1 einige grundlegende Einflussfaktoren thematisieren, die die Mechanismen der Evokation eines Konstruktions-Frames steuern. Dazu gehört zunächst die Unterscheidung der Evokation eines Konstruktions-Frames von derjenigen eines lexikalischen Frames, bevor noch einmal zwei semantische Parameter von Konstruktionen, Frame-Nähe und formale Abstraktheit, betrachtet werden müssen. Die beiden folgenden Abschnitte nehmen dann für die drei untersuchten Konstruktionen Mechanismen der Evokation des Konstruktions-Frames Motion

2 Die Frage nach der Evokation eines Frames stellt Ziem (2008: 231-237, 2014b: 197-202) explizit unter den Oberbegriff der Relation. Zu letzterem Terminus schreibt er: „Der Begriff ,Relation“ [...] ist hier nur eine Metapher für einen inferentiellen Prozess, den die phonologische Einheit eines Sprachzeichens bei einem verstehenden Sprachbenutzer oder einer verstehenden Sprachbenutzerin in Gang setzt.“ (Ziem 2008: 231). Auf die kognitionstheoretischen und psycholinguistischen Details solcher Inferenzprozesse gehe ich allerdings nicht ein. 
in ihren Konstrukten in den Blick. Eine wesentliche Rolle spielt dabei das Konzept der Frame-Nähe (Abschnitt 5.4) mit der Unterscheidung in relatierte und unrelatierte lexikalische Frames. In Abschnitt 8.2 steht die Situation, in der ein Konstrukt-Frame ausschließlich aus FE eines relatierten lexikalischen Frames besteht (Unterabschnitt 6.2.1), im Vordergrund, während es in Abschnitt $8.3 \mathrm{um}$ diejenigen Konstrukte gehen soll, deren Konstrukt-Frames durch einen Blend aus lexikalischem Frame und Konstruktions-Frame zustande kommen (vgl. dazu Unterabschnitt 4.1.3). Mit methodischen Fragen nach der Ermittlung eines Konstruktions-Frames für eine Konstruktion möchte ich den Abschluss dieses Kapitels bilden. In Abschnitt 8.4 soll es um einige methodologische Präliminarien gehen, die Vorarbeiten zur Anwendung unterschiedlicher Methoden zur Ermittlung des Konstruktions-Frames liefern. In Abschnitt 8.5 schließlich möchte ich drei solcher Methoden diskutieren, die sich mit unterschiedlichem empirischen Nutzen auf die drei untersuchten Konstruktionen anwenden lassen.

\subsection{Einflussfaktoren bei der Evokation eines Konstruktions-Frames}

Die Frage nach dem Mechanismus, durch den eine sprachliche Einheit mit einem Frame assoziiert wird, beantwortet Fillmore (1982a: 117) mit der berühmt gewordenen Faustregel, „that the word 'evokes' the frame.“ Seitdem ist die Redeweise des ,Evozierens‘ eines Frames durch eine LE fester Bestandteil der - traditionell nur auf LE als Target-Einheiten beschränkten - Frame-Semantik. Ein Frame wird immer dann evoziert, wenn die betreffende LE in einem konkreten Ko(n)text, sei es innerhalb eines Konstrukts einer syntaktischen Konstruktion, auftritt. Möchte man wissen, wann ein gegebener Frame evoziert wird, reicht es aus rein lexikalischer Perspektive demnach aus, sich all diejenigen LE anzusehen, die hierfür infrage kommen - entsprechend werden diese in FrameNet zu den dort beschriebenen Frames dokumentiert (vgl. Unterabschnitt 2.1.2).

Bleibt man gemäß des konstruktionssemantischen Modells (Kapitel 4) zunächst bei lexikalischen Frames, ist es also (scheinbar) unproblematisch, den Frame zu identifizieren, der durch eine LE identifiziert wird. Genau dies definiert lexikalische Frames: Sie werden von einer LE evoziert, die als KtE das KE einer Konstruktion instanziieren kann (vgl. Abschnitt 4.2). Es handelt sich dabei um eine symbolische Assoziation im Sinne von Schmid (2020: 46), in der eine sprachliche Form (eben eine LE) mit einem Frame assoziiert ist. Stellt man sich nun aber die Frage, wie ein Konstruktions-Frame evoziert wird, so ergeben sich Schwierigkeiten, vor allem weil unklar ist, ob die Relation zwischen einer Konstruktion und 
einem Frame ebenso beschaffen ist wie diejenige zwischen einer LE und einem Frame. Gerade FrameNet - und in großen Teilen die Frame-Semantik insgesamt - ist im Kern lexikalisch ausgerichtet: Es ist in erster Linie ein Unterfangen zur Beschreibung lexikalischer Frames (vgl. Unterabschnitt 2.1.3). ${ }^{3}$ Vor dem Hintergrund der Geschichte der Frame-Semantik mag dies verwundern, denn wie Busse (2012: 658-659) resümiert, ist die Reduktion frame-semantischer Phänomenbereiche auf LE eine vergleichsweise späte Entwicklung, die erst nach dem ursprünglichen Interesse an komplexeren sprachlichen Ausdrücken, Sätzen und Texten, eingesetzt hat. ${ }^{4}$ Auch wenn dies aus konstruktionsgrammatischer und konstruktikographischer Sicht bemerkenswert ist, zeigt die frühe Fokussierung auf Sätze und Texte, dass komplexere sprachliche Ausdrücke - wenn überhaupt - eher auf Token- und nicht auf Type-Ebene in den Blick genommen wurden. Wenn Busse (2012: 659) also für eine „Analyse der Frame-aktivierenden Leistungen von LexemKetten" plädiert, fallen abstraktere syntaktische Konstruktionen als Einheiten auf Type-Ebene, ${ }^{5}$ zu denen die reflexive Bewegungskonstruktion, die reflexive Partikelverbkonstruktion und die reflexive Weg-Konstruktion zählen, aus dem Raster. Erst mit dem Einsetzen der konstruktikographischen Forschung wird dieser Mangel, wie erstmals Fillmore (2008a) eindrücklich schildert, explizit adressiert, allerdings mit der ungewollten Konsequenz, dass - wie in Unterabschnitt 2.3.2 zusammengefasst - Frames zur Beschreibung der semantischen Eigenschaften von Konstruktionen bisher eher spärlich herangezogen werden.

Worin liegen die Ursachen für dieses Defizit? Zum einen fehlt es an einer analogen Dokumentation von (syntaktischen) Konstruktionen, die einem Frame zugeordnet werden könnten: FrameNet als lexikalische Datenbank scheint hierfür auf den ersten Blick nicht zuständig (vgl. Hasegawa, Lee-Goldman \& Fillmore 2014: 198). ${ }^{6}$ Zum anderen fehlt es nicht nur an Methoden, den als Konstruktions-Frame anzusehenden Frame zu identifizieren (worauf ich in den Abschnitten 8.4 und 8.5 zurückkomme), sondern es fehlt ebenso an einer theoretischen Vorstellung der Mechanismen, durch die eine Konstruktion einen Konstruktions-Frame evozieren

3 Dieser Tatsache war sich schon Fillmore (1982a: 117) bewusst, wenn er die obige Faustregel auf die Tatsache „that the frame structures the word-meanings“ bezieht.

$4 \mathrm{Zu}$ erkennen ist dies an Titeln früherer Arbeiten wie etwa Fillmore (1982c, 1984).

5 Nur ganz am Rande erwähnt Busse (2012: 661, Anm. 219) die mit Konstruktionen grundsätzlich zu vergleichenden traditionellen Satzbaupläne (vgl. dazu Höllein 2019: 57-60; Ágel \& Höllein 2021: 134-140), bleibt aber grundsätzlich bei der frame-evozierenden Leistung einzelner Verben als Prädikate stehen.

6 Vgl. aber die Diskussion in Unterabschnitt 2.1.3 sowie die von Ziem (2015d: 72, Anm. 30) aufgezeigten Gegenbeispiele. 
kann. ${ }^{7}$ Es scheint zumindest intuitive Evidenz dafür zu geben, dass ,[f]rames may also have a conventional connection to a simple syntactic construction or idiom“ (Gawron 2011: 670). Bisweilen erscheint die Evokation von Frames durch Konstruktionen dabei vollkommen parallel zu derjenigen von LE verstanden zu werden, wenn etwa Croft \& Cruse (2004: 8) schreiben: „[W]ords and constructions evoke an understanding, or more specifically a frame; a hearer invokes a frame upon hearing an utterance in order to understand it." ${ }^{8}$ Ebenso betont Ziem (2018c: 122): „Wichtig zu sehen ist, dass (auch abstrakte) grammatische Konstruktionen - wie lexikalische Einheiten - Frames evozieren.“

Aus konstruktikographischer Sicht hat dies im Falle des Konstruktikons für das Brasilianische Portugiesisch gar zur Postulation zusätzlicher Entitäten - Konstruktionseinheiten (constructional units, CUs) - geführt, die analog zu LE einem Frame zugeordnet werden, um zu dokumentieren, dass dieser Frame durch bestimmte Konstruktionen evoziert wird (vgl. Torrent et al. 2014: 45). Wie in Unterabschnitt 2.3.2 gesehen, weisen Fillmore, Lee-Goldman \& Rhomieux (2012: 324-330) einen eigenen Typ der frame-evozierenden Konstruktionen aus, zu denen sie die way-Konstruktion zählen. Aufbauend auf die Dichotomie frame-evozierender und nicht frame-evozierender Konstruktionen von Lyngfelt et al. (2018: 69-81) differenziert Ohara (2018: 151-160) insgesamt fünf Typen von Konstruktionen, die sich in diese Dichotomie einordnen lassen (vgl. Tabelle 2.5 in Unterabschnitt 2.3.2).

Gerade aufgrund der oft angenommenen parallelen frame-evozierenden Leistung von LE und Konstruktionen muss die Frage nach möglichen Unterschieden zwischen der Evokation eines lexikalischen Frames und derjenigen eines Konstruktions-Frames der Diskussion um Mechanismen Letzter vorgeschaltet sein. Aus diesem Grund beginne ich diesen Abschnitt zu möglichen Einflussfaktoren solcher Mechanismen mit jenem Aspekt des Unterschieds zwischen der Evokation eines lexikalischen Frames und derjenigen eines Konstruktions-Frames in Unterabschnitt 8.1.1. Im darauffolgenden Unterabschnitt 8.1.2 möchte ich dafür argumentieren, dass die Frage nach dem Mechanismus der Evokation des

7 Wie Busse (2012: 669) kritisiert, besteht die theoretische Lücke, was unter ,Evozieren` konkret zu verstehen ist, bereits in Fillmores Verstehenssemantik. Sie überträgt sich also auf die Konstruktionsgrammatik und Konstruktikographie, zumindest solange, wie diese Ansätze Anschluss an eines der Frame-Modelle Fillmores suchen.

8 Croft \& Cruse (2004: 8) rekurrieren hiermit auf die von Fillmore (1982a: 124, 1985a: 232, 1986a: 53) eingeführte Dichotomie zwischen dem Evozieren und Invozieren eines Frames: Ersteres geschieht durch einen sprachlichen Ausdruck, Letzteres ist die Leistung der Rezipierenden. Vgl. dazu auch Ziem (2008: 231-237, 2014b: 197-202). Ich komme auf diese Dichotomie in Unterabschnitt 8.1.1 zurück, spreche aber bereits jetzt ausschließlich von Evokation, da ich mich grundsätzlich der Kritik von Busse (2012: 122-131, 667-670) anschließe, der zahlreiche verstehenstheoretische Probleme der Unterscheidung zwischen Evokation und Invokation aufzeigt. 
Konstruktions-Frames stark von der Konstitution des Konstrukt-Frames abhängt, genauer: dem lexikalischen Frame, der in den Konstrukt-Frame eingeht. Dort wird also noch einmal auf den Begriff der Frame-Nähe und die Unterscheidung in relatierte und unrelatierte lexikalische Frames einzugehen sein. Dieser Argumentation liegt die Annahme zugrunde, dass es für ein und dieselbe Konstruktion bisweilen unterschiedliche Varianten der Evokation eines Konstruktions-Frames geben kann, die sich nach der Frame-Nähe lexikalischer Frames bemessen lassen. Darüber hinaus lässt sich aber mindestens ein weiterer Einflussfaktor auf die Mechanismen der Evokation eines Konstruktions-Frames ermitteln, der sich an den semantischen Parameter der formalen Abstraktheit einer Konstruktion (Abschnitt 5.1) zurückbinden lässt. Auf diesen gehe ich in Unterabschnitt 8.1.3 ein. ${ }^{9}$

\subsubsection{Evokation lexikalischer Frames vs. Evokation von Konstruktions-Frames}

Obwohl die Frame-Semantik, wie soeben dargestellt, traditionell davon ausgeht, dass in erster Linie LE Frames evozieren, wird parallel, ähnlich wie Hilpert (2009: 38) es tut, bisweilen mit einer gewissen Selbstverständlichkeit davon ausgegangen, dass auch syntaktische Konstruktionen dazu in der Lage sind: „There are also grammatical constructions which evoke semantic, pragmatic, or interactional frames on their own." (Fillmore \& Baker 2010: 338). Die zum Eingang dieses Abschnitts zusammengestellten Zitate vervollständigen dieses Bild. Es stellt sich allerdings die Frage, ob sich und wenn ja, was, eine solche Konstruktion hinsichtlich der Evokation eines Konstruktions-Frames von einer LE und der Evokation eines lexikalischen Frames unterscheidet.

In Unterabschnitt 6.2.1 habe ich für die reflexive Bewegungskonstruktion gezeigt, dass für zahlreiche ihrer Konstrukte eine Identität zwischen KonstruktionsFrame und lexikalischem Frame belegt ist, in denen also Motion als lexikalischer

9 Einen weiteren potenziellen Einflussfaktor nennt Ziem (2020a: 32): „Eine Konstruktion kann [...] nur dann einen Frame aufrufen, wenn sich zumindest ihre Kern-KE auf die Kern-FrameElemente (FE) des Frames abbilden lassen. “ Für die drei untersuchten Konstruktionen stellt sich diese Frage nach den Strukturparallelen zwischen Konstruktionen und Frames allerdings nicht, da die Motivierung von KtE durch FE zumindest bei relatierten lexikalischen Frames immer durch einen dem Konstruktions-Frame nahestehenden (lexikalischen) Frame gegeben ist. Bei unrelatierten lexikalischen Frames liegen indes weitere Evokationsmechanismen vor, die potenziell unabhängig von den Strukturparallelen operieren (vgl. Abschnitt 8.3). Es ist jedoch freilich denkbar, dass sich dies bei anderen Typen von Konstruktionen als den drei hier untersuchten anders darstellt, sodass es lohnenswert wäre, den von Ziem genannten Einflussfaktor weiter zu untersuchen. 
Frame erscheint, was zur Folge hat, dass alle KtE eines solchen Konstrukts einfach durch FE ebendieses Frames motiviert werden. Auch für die anderen beiden Konstruktionen ist dieser Fall belegt. Die Belege in (1), (2) und (3) sind noch einmal einige Beispiele für Motion als lexikalischen Frame in den drei untersuchten Konstruktionen.

(1) a. JOHANNES PAUL reiste nach Madaba und [Motion begab] sich [GoAL auf den Berg Nebo, wo nach der Überlieferung der Bibel Moses das gelobte Land erschaut haben soll]. (Archiv der Gegenwart, 2001 [2000])

b. Nachdem er ausgestiegen war, [Motion bewegte] sich [Theme Pardell] aufmerksam und schnell [РАтн durch den dichten Schneefall auf dem Paß]. (Kopetzky, Steffen: Grand Tour, Frankfurt am Main: Eichborn 2002, S. 714)

c. [Theme Er] [Motion rückt] sich [GoAL in den Mittelpunkt]. (Schwanitz, Dietrich: Männer, Frankfurt a. M.: Eichborn 2001, S. 73)

(2) a. Die Karkasse war an ihrer Unterseite aufgerissen, [Tнеме die Eingeweide] waren hervorgequollen, [Motion schlängelten] sich [Dівестіon auf] und dümpelten im Wasser zwischen den Algen und dem Kelp. (Schrott, Raoul: Tristan da Cunha oder die Hälfte der Erde; Hanser Verlag 2003, S. 72)

b. Sie verspricht es, und [Theme ich] [Motion schlängele] mich unauffällig [РАтн zwischen anderen Gästen] [DiRECTion hindurch], bis ich den geheimnisvollen Salon erreiche. (Noll, Ingrid: Ladylike, Zürich: Diogenes 2006, S. 75)

c. Seine Scheunenvierteluhr war entworfen, und [Tнеме eine Raupe aus grauen, Gestalten] [Motion wand] sich bei Sonnenuntergang, schlurfend, schweigend, [РАтн am Schloßberg] [Direction hoch]. (Koneffke, Jan: Paul Schatz im Uhrenkasten, Köln: DuMont Buchverlag 2000, S. 228)

(3) a. Was passiert ist, ist passiert“, und er drehte sich um und [Motion bahnte] sich langsam [РАTH durch die Büsche] seinen Weg [GoAL zurück zum Gutshaus]. (Boie, Kirsten: Skogland, Ort: Hamburg 2005, S. 185)

b. [Theme Paul] mußte sich einen Pfad [GoAL zum Holzhaus] [Motion bahnen]. (Koneffke, Jan: Paul Schatz im Uhrenkasten, Köln: DuMont Buchverlag 2000, S. 77)

c. Jawohl, denn die Damen wirken „schwerfällig und lustlos“, [Motion bahnen] sich an Bord nur mühsam ihren Weg [РАтн durch die engen Gänge] und ecken sehr wahrscheinlich auch bei solchen Passagieren an, die 
es in höchstem Maße unerquicklich finden, ihren Anisschnaps von einem Fesselballon serviert zu bekommen. (Die Zeit, 10.02.2000, Nr. 7)

Solche Konstrukte, in denen der lexikalische Frame direkt dem KonstruktionsFrame Motion entspricht, bilden den einzigen Fall, in dem Letzterer vollkommen analog zu lexikalischen Frames evoziert wird. Nicht umsonst ist Motion der prototypischste aller (relatierten) lexikalischen Frames, da er mit dem KonstruktionsFrame identisch ist (vgl. Unterabschnitt 5.4.2). In seiner Rolle als lexikalischer Frame wird er durch eine als KtE des KE EREIGNIS instanziierte LE evoziert, ebenso wie dies für alle anderen lexikalischen Frames der Fall ist. Die Evokation des Konstruktions-Frames ist somit als Kontinuum zu verstehen, auf dem das eine Extrem eine Evokation analog zu derjenigen lexikalischer Frames bildet und das andere Extrem eine maximal davon zu unterscheidende Evokation, die derjenigen eines genuinen Konstruktions-Frames entspricht, der nicht mit einem lexikalischen Frame identisch ist und zu dem ein gegebener lexikalischer Frame unrelatiert ist. Ein solches Kontinuum ist in Abbildung 8.1 dargestellt, wobei der soeben an den Belegen in (1)-(3) illustrierte Fall auf der linken Seite zu verorten ist.

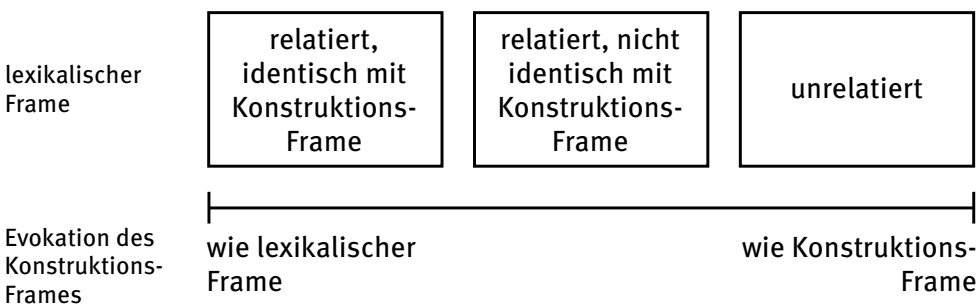

Abb. 8.1: Kontinuum der Evokation eines Konstruktions-Frames

Nun bilden, wie in Unterabschnitt 6.2.1 für die reflexive Bewegungskonstruktion und in Unterabschnitt 6.4.2 für die reflexive Partikelverbkonstruktion gesehen, Konstrukte mit Motion als prototypischstem aller lexikalischen Frames jedoch nur einen Teil aller Daten für beide Konstruktionen. Das andere Extrem, das in Abbildung 8.1 auf der rechten Seite verortet ist, bilden diejenigen Konstrukte, in deren Konstrukt-Frames unrelatierte lexikalische Frames eingehen, die in keiner FrameNähe zu Motion als Konstruktions-Frame stehen. Die folgenden Belege in (4)-(6) sind Beispiele für solche Fälle der reflexiven Bewegungskonstruktion, der reflexiven Partikelverbkonstruktion und der reflexiven Weg-Konstruktion.

(4) a. [Елтіту Die dritte Schöpfung indes, die parallele Welt der ewig laufenden Fernsehfilme, der Kino-Genres und Star-Träume, der Virtual Reali- 
ty in den Computern], [Motion entwickelt] [sich ${ }^{\text {THEME}}$ ] genauso konstant (und genauso bedrohlich) [in die Vergangenheit zurück ${ }^{\text {THEME }}$. (Die Zeit, 10.02.2000, Nr. 7)

b. [agent Spinnenweb] [attaching klebte] [IтEm sich ${ }^{\text {ThEmE }}$ [ [Goal in sein Gesicht ${ }^{\text {GoAL }}$ ]. (Koneffke, Jan: Paul Schatz im Uhrenkasten, Köln: DuMont Buchverlag 2000, S. 216)

c. Die Häuser biegen sich vor Verzweiflung, an den Fenstern treiben Rauchfetzen vorbei, „groß wie junge Katzen“, [Patient die Tapeten]

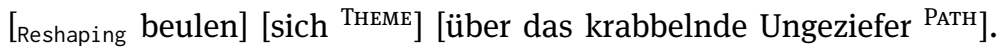
(Die Zeit, 13.04.2000, Nr. 16)

(5) a. Mit einer Nadel kratzt man hierauf die Konturen durchs Wachs und gießt Säure in diese fast unsichtbaren Furchen, [Agent die] [Material

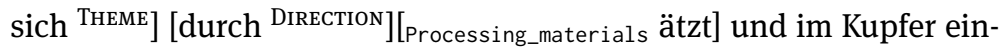
brennt, die schwarze giftige Galle meiner Melancholie, mit der die wahren Umrisse der Welt einem so schmerzhaft anschaulich werden. (Schrott, Raoul: Tristan da Cunha oder die Hälfte der Erde; Hanser Verlag 2003, S. 133)

b. Sie sprachen jenes flüssige, großzügig dem Englischen entliehene uni-

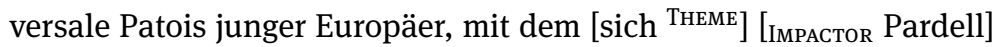
selbst meistens [durch Direction][Impact schlug], wenn er nicht sein rührendes, vor lauter Umständlichkeit manchmal für Momente erstarrendes Französisch ausprobierte. (Kopetzky, Steffen: Grand Tour, Frankfurt am Main: Eichborn 2002, S. 230)

c. Mit den Jahrzehnten des Erfolgs, mit jeder geglückten Erweiterung [Fire_burning brannte] [sich THEME] [FIRE ein Denkmuster] bei Politikern verschiedenster Couleur und Nation tiefer [ein Direction]. (Die Zeit, 20.01.2000, Nr. 4)

(6) Mein Schälmesser mit der dünnen Klinge [cutting säbelt] sich einen Weg [durch die buschigen Petersilienköpfe ${ }^{\text {РATH}}$ ], während ich überlege, ob es tatsächlich Köpfe oder doch Blätter oder gar Büschel heißt. (Riedel, Susanne: Eine Frau aus Amerika, Berlin: Berlin Verlag 2003, S. 106)

Vergleicht man die Konstrukte in (1)-(3) mit Motion als lexikalischem Frame mit denjenigen in (4)-(6) mit Motion als Konstruktions-Frame und einem unrelatierten lexikalischen Frame, so lassen sich einige Unterschiede in der Evokation eines Konstruktions-Frames im Gegensatz zu derjenigen eines lexikalischen Frames herausarbeiten, die sich insbesondere an Konstrukten mit unrelatierten lexikalischen Frames beobachten lassen. Für unrelatierte lexikalische Frames sind zwei wesentliche Beobachtungen festzuhalten. 
1. Der Konstruktions-Frame wird nicht durch ein einzelnes Strukturelement eines Konstrukts evoziert, also nicht etwa durch eine als KtE eines KE (z.B. EREIGNIS) instanziierte LE, die als Motion evozierend betrachtet werden könnte.

2. Der Konstruktions-Frame wird im Falle der reflexiven Bewegungskonstruktion und der reflexiven Partikelverbkonstruktion nicht durch ein lexikalisch fixiertes Element, also ein KEE oder KorE, evoziert. Die reflexive WegKonstruktion bildet hier eine Ausnahme (vgl. Unterabschnitte 8.1.3 und 8.3.2).

Konstrukte, in deren Konstrukt-Frames unrelatierte lexikalische Frames eingehen, stellen somit besondere Herausforderungen an eine Vorstellung davon, wie der Konstruktions-Frame in solchen Konstrukten evoziert werden kann. Gerade bei Argumentstruktur-Konstruktionen wie den drei untersuchten dürften diese Fälle, in denen der Konstruktions-Frame nicht zwangsläufig von einer instanziierten LE in einem Konstrukt evoziert werden muss, häufiger auftreten. Gerade die zweite der obigen Beobachtungen, die insbesondere formal abstrakte syntaktische Konstruktionen betrifft, die über kein oder nur wenig lexikalisch spezifiziertes Material in Form von KEE oder KorE verfügen, stellt eine Herausforderung für die Ermittlung des Konstruktions-Frames, den diese Konstruktionen evozieren, dar. Aus diesem Grund wird der semantische Parameter der formalen Abstraktheit einer Konstruktion in diesem Zusammenhang noch eine Rolle spielen. Diese ersten Beobachtungen sind zusammengenommen Evidenz dafür, dass Konstruktionen zumindest in einer Teilmenge ihrer Konstrukte - ihren Konstruktions-Frame nicht auf dieselbe Art und Weise wie LE evozieren:

[T] here are various cxns being more or less related to frames in different ways, such as imperative cxns, which, while obviously related to the Request frame, hardly evoke it the same way words like order and command do; rather than referring to requests, like these words do, imperative cxns are used to perform them. (Boas, Lyngfelt \& Torrent 2019: 47)

Wird ein Konstruktions-Frame also, in Fällen, die unter den zweiten der obigen Punkte fallen, nicht durch eine im Konstrukt (als KtE, KEE oder KorE) realisierte LE des betreffenden Frames evoziert, wirft dies die Frage nach der Natur der Evokation des Konstruktions-Frames in solchen Konstrukten auf. Unterscheidet sie sich von einer ,lexikalischen' Evokation wie derjenigen, die in Abbildung 8.1 auf der linken Seite zu sehen ist? Und wenn ja, wie? In seiner VerstehenssemantikPhase hat Fillmore diese (scheinbare) Unterscheidung in dem Mechanismus der Evokation eines Frames mit der Dichotomie von Evozieren und Invozieren zu begegnen versucht. Er begründet sie wie folgt:

On the one hand, we have cases in which the lexical and grammatical material observable in the text 'evokes' the relevant frames in the mind of the interpreter by virtue of the fact that 
these lexical forms or these grammatical structures or categories exist as indices of these frames; on the other hand, we have cases in which the interpreter assigns coherence to a text by 'invoking' a particular interpretive frame. (Fillmore 1982a: 124)

An anderer Stelle nuanciert Fillmore diese Unterscheidung etwas anders:

\begin{abstract}
Interpretive frames can be introduced into the process of understanding a text through being invoked by the interpreter or through being evoked by the text. A frame is invoked when the interpreter, in trying to make sense of a text segment, is able to assign it an interpretation by situating its content in a pattern that is known independently of the text. A frame is evoked by the text if some linguistic form or pattern is conventionally associated with the frame in question. (Fillmore 1985a: 232)
\end{abstract}

An wiederum anderer Stelle bringt Fillmore (1986a: 53) das Verhältnis auf folgende Faustformel: „The interpreter of a text invokes frames, segments of the text evoke frames. “10

Man könnte also zu der Schlussfolgerung kommen, dass lexikalische Frames und auch der Konstruktions-Frame, wenn er mit dem lexikalischen Frame identisch ist, evoziert wird, während der Konstruktions-Frame - sofern der im Konstrukt-Frame hinzukommende lexikalische Frame unrelatiert ist - invoziert wird. Obwohl Fillmore (1982a: 124, 1985a: 232) den Mechanismus des Evozierens auch grammatischen Mustern (oder eben: Konstruktionen) zuschreibt, ist es keineswegs so, dass allein eine Konstellation von KtE, KEE und KorE in der Lage ist, einen Konstruktions-Frame zu evozieren. Die im Kontinuum der Evokation eines Konstruktions-Frames (Abbildung 8.1) links verorteten Konstrukte sind ja Evidenz dafür, dass ein Konstruktions-Frame, wenn er mit dem lexikalischen Frame identisch ist, gleichermaßen ,lexikalisch' evoziert werden kann.

Warum also ist es nicht sinnvoll, vom Evozieren eines lexikalischen Frames und vom Invozieren eines Konstruktions-Frames zu sprechen? Wie Busse (2012: 123-131) ausführlich zeigt, ist die Unterscheidung zwischen Evozieren und Invozieren sprach- und kognitionstheoretisch problematisch, unter anderem weil es in jedem Fall die Interpretierenden sind, die ein Konstrukt, einen Satz oder einen Text ,bedeutungshaltig' machen, nicht das sprachliche Zeichen selbst. ${ }^{11}$ Streng genommen müsste man also stets von Invozieren sprechen, dennoch hat sich der Terminus Evozieren als allgemeinerer Ausdruck etabliert. ${ }^{12}$ Genauer betrachtet

10 Vgl. auch die ähnliche Definition in Fillmore \& Baker (2010: 316).

11 Vgl. aber die Ausführungen von Ziem (2008: 231-237, 2014b: 197-202), der die Begriffe des Evozierens und Invozierens mit Aufrufen und Abrufen übersetzt und anhand dieser Begriffe für eine Unterscheidung plädiert.

12 Wie Busse (2012: 203-209) nachweist, werden auch bei FrameNet bisweilen beide Begriffe benutzt, ohne diese jedoch terminologisch zu differenzieren. 
kann nun auch die Einschränkung, dass grammatische Muster, im vorliegenden Fall also Konstruktionen, streng genommen keine Frames evozieren, sondern nur invozieren können, aufgehoben werden: Konstruktionen können sehr wohl Konstruktions-Frames evozieren, die Einschränkung muss lediglich lauten: Nicht alle Konstrukte tun dies, wie im Kontinuum der Evokation eines KonstruktionsFrames in Abbildung 8.1 dargestellt, auf dieselbe Art und Weise.

Was bei der Untersuchung der Mechanismen der Evokation von KonstruktionsFrames berücksichtigt werden muss, sind nicht die (vermeintlichen) Unterschiede zwischen einem Evozieren und einem Invozieren, sondern die unterschiedlichen Voraussetzungen, die die Konstrukte einer Konstruktion erfüllen, um einen Konstruktions-Frame zu evozieren. Einige Voraussetzungen habe ich bereits herausgearbeitet: Konstrukte, in denen der lexikalische Frame dem KonstruktionsFrame entspricht, unterscheiden sich wesentlich von solchen, in denen der lexikalische Frame zum Konstruktions-Frame unrelatiert ist. Zwischen diesen beiden Extremen der Identität zwischen lexikalischem Frame und Konstruktions-Frame auf der einen Seite und unrelatierten lexikalischen Frames auf der anderen Seite liegen freilich relatierte lexikalische Frames, die nicht mit dem Konstruktions-Frame identisch sind, also all solche, die über eine Frame-Nähe ungleich 0 verfügen (vgl. das System der Frame-Nähen von Mot ion in Unterabschnitt 5.4.3). In Abbildung 8.1 stellen sie die Mitte des Kontinuums der Evokation eines Konstruktions-Frames dar. Dies deutet darauf hin, dass die Frame-Nähe lexikalischer Frames und lexikalische Frames allgemein einen wesentlichen Einflussfaktor für den Mechanismus der Evokation eines Konstruktions-Frames darstellen. Bevor ich darauf im folgenden Unterabschnitt 8.1.2 eingehe, ist festzuhalten, dass die Frage nach dem Mechanismus der Evokation des Konstruktions-Frames für eine gegebene Konstruktion nicht invariant beantwortet werden kann. Je nach auf lexikalische Frames bezogener Konstitution eines Konstrukt-Frames, deren drei Varianten in Abbildung 8.1 dargestellt sind, kann es unterschiedliche Varianten der Evokation eines Konstruktions-Frames geben.

\subsubsection{Noch einmal: lexikalische Frames und Frame-Nähe}

Nicht erst das Kontinuum der Evokation des Konstruktions-Frames, das ich in Abbildung 8.1 skizziert habe, hat es bereits angedeutet: Lexikalische Frames und insbesondere deren Frame-Nähe (Abschnitt 5.4) spielen eine zentrale Rolle bei der Frage, welchem Mechanismus die Evokation eines Konstruktions-Frames folgt. Deutlich geworden ist dies bereits im Zuge der Diskussion der variierenden Motivierung von KtE und KEE durch FE des Konstruktions-Frames in Kapitel 6. Die Ergebnisse dort zeigen, dass der Anteil des Konstruktions-Frames an einem 
Konstrukt-Frame und damit die Evokation des Letzteren von einem wesentlichen Faktor abhängt: dem lexikalischen Frame. Ist der lexikalische Frame in der Lage, alle KtE und KEE semantisch zu motivieren, wird der Konstruktions-Frame kaum unabhängig von diesem lexikalischen Frame evoziert. Die Antwort auf die Frage, nach welchen Mechanismen der Konstruktions-Frame evoziert wird, muss also in einem entscheidenden Maße an lexikalischen Frames ausgerichtet werden.

Die Fälle, in denen lexikalischer Frame und Konstruktions-Frame identisch sind, in denen also ein KtE des KE EREIGNIS direkt Motion evoziert (vgl. die Beispiele in Unterabschnitt 8.1.1), sind Evidenz dafür, dass es grundsätzlich möglich ist, dass der Konstruktions-Frame analog zu einem lexikalischen Frame über ein ebensolches KtE evoziert werden kann. Das Ausmaß, bis zu dem dies möglich ist, wird über den entsprechenden lexikalischen Frame und dessen Frame-Nähe zum Konstruktions-Frame bestimmt. Die Frame-Nähe des lexikalischen Frames und dessen Prototypikalität in Bezug auf den Konstruktions-Frame (Unterabschnitt 5.4.2) entscheidet maßgeblich darüber, wie stark die Evokation des KonstruktionsFrames ausfällt (dazu Unterabschnitt 8.2.2). Das Kriterium der Frame-Nähe eines lexikalischen Frames deutet darauf hin, dass nur eine Klasse von Frames für die Evokation des Konstruktions-Frames über eine KtE des KE EREIGNIS infrage kommt: relatierte lexikalische Frames. Bei unrelatierten lexikalischen Frames muss es immer einen anderen Mechanismus der Evokation des KonstruktionsFrames geben, in Konstrukten mit unrelatierten lexikalischen Frames kann der Konstruktions-Frame nicht (auch nicht indirekt) über eine KtE des KE EREIGNIS evoziert werden.

Die relatierten lexikalischen Frames, die für die Evokation des KonstruktionsFrames über ein KtE des KE EREIGNIS infrage kommen, zerfallen wiederum in zwei Klassen (vgl. Unterabschnitte 5.4.2 und 7.5.1):

1. den lexikalischen Frame, der mit dem Konstruktions-Frame identisch ist;

2. alle anderen relatierten lexikalischen Frames, die zum Konstruktions-Frame in einer Frame-Nähe ungleich 0 stehen.

Den Fall, in dem lexikalischer Frame und Konstruktions-Frame identisch sind, habe ich soeben in Unterabschnitt 8.1.1 betrachtet, sodass ich diese Analyse hier nicht wiederhole. Stattdessen sei der Blick auf diejenigen relatierten lexikalischen Frames gerichtet, die nicht mit dem Konstruktions-Frame identisch sind.

Die drei grundlegenden Varianten des Anteils des Konstruktions-Frames Motion an der Konstitution von Konstrukt-Frames und der semantischen Motivierung von KtE und KEE, die ich in den Abschnitten 6.2 und 6.3 für die reflexive Bewegungskonstruktion aufgezeigt habe (einfache Motivierung durch lexikalischen Frame, einfache Motivierung durch Konstruktions-Frame, doppelte Motivierung), zeigen, dass der Konstruktions-Frame eine verschieden gewichtige Rolle inner- 
halb der Konstrukte einer Konstruktion spielen kann. Auch die Unterscheidung der sieben Koerzionsstufen, die ich für die Messung des Koerzionspotenzials der drei untersuchten Konstruktionen aufgestellt habe (vgl. Unterabschnitte 7.4.1 und 7.4.3) spiegelt diese Varianz wider. Je höher die Koerzionsstufe und je stärker damit die Frame-Anpassung des lexikalischen Frames durch den KonstruktionsFrame (Unterabschnitt 4.4.1), desto höher der Anteil des Konstruktions-Frames an der Konstitution von Konstrukt-Frames und der semantischen Motivierung von KtE und KEE. Die Feststellung dieser Varianz im Anteil des KonstruktionsFrames an Konstrukt-Frames muss zu der Annahme führen, dass auch dessen Evokation einer Varianz über die Konstrukte einer Konstruktion hinweg unterworfen ist. Über den Anteil des Konstruktions-Frames an einem Konstrukt-Frame entscheidet in erster Linie der lexikalische Frame, also zum einen, ob es sich um einen zum Konstruktions-Frame relatierten oder unrelatierten Frame handelt und zum anderen die Prototypikalität relatierter Frames (Unterabschnitt 5.4.2). Kurzum: Wenn der Anteil des Konstruktions-Frames am Konstrukt-Frame wesentlich durch das Konzept der Frame-Nähe lexikalischer Frames determiniert wird, muss diese Frame-Nähe ein wesentlicher Einflussfaktor dafür sein, wie der Konstruktions-Frame in einem Konstrukt evoziert wird.

\subsubsection{Noch einmal: formale Abstraktheit einer Konstruktion}

In einer Argumentstruktur-Konstruktion wie der reflexiven Bewegungskonstruktion, der reflexiven Partikelverbkonstruktion oder der reflexiven Weg-Konstruktion ist die Evokation eines lexikalischen Frames im Gegensatz zu derjenigen des Konstruktions-Frames deutlich restringierter und im Wesentlichen auf einen einzigen Mechanismus reduziert. Ein lexikalischer Frame wird, wie in Abschnitt 4.2 definiert, von einer als KtE eines KE instanziierten LE evoziert. Für die drei untersuchten Konstruktionen kommt hierfür nur ein einziges KE infrage: EREIGNIS. Dies ist der Grund, warum dieses KE als einziges nicht im Rückgriff auf FE benannt und definiert werden kann, sondern dies auf Grundlage von Frames als Ganzes geschehen muss (vgl. Unterabschnitt 7.3.1). Daraus folgt, dass für die Evokation des lexikalischen Frames nur dieses eine Strukturelement, das KE EREIGNIS, infrage kommt.

Die Evokation des Konstruktions-Frames ist jedoch, zumindest für die drei untersuchten Konstruktionen, nicht in dieser Weise restringiert. Dies ist bereits daran zu erkennen, wenn, wie in Unterabschnitt 8.1.1 festgestellt, dessen Evokation vollkommen analog zu derjenigen eines lexikalischen Frames geschehen kann, wenn Konstruktions-Frame und lexikalischer Frame identisch sind. Sind beide nicht identisch und evoziert die LE, die als KtE des KE EREIGNIS instanziiert wird, 
einen anderen relatierten oder einen unrelatierten lexikalischen Frame (im Kontinuum der Evokation eines Konstruktions-Frames in Abbildung 8.1 sind diese Fälle in der Mitte und rechts verortet), muss die Evokation des Konstruktions-Frames durch andere Mechanismen beeinflusst werden. Die Tatsache, dass ein lexikalischer Frame immer über das KtE eines bestimmten KE evoziert wird, die Evokation des Konstruktions-Frame hingegen über weitere Mechanismen verlaufen muss, deutet darauf hin, dass neben der Frame-Nähe des lexikalischen Frames (Unterabschnitt 8.1.2) ein zweiter Faktor über den Mechanismus der Evokation des Konstruktions-Frames entscheidet: die formale Abstraktheit der Konstruktion. Die formale Abstraktheit der Konstruktion ist wie die Frame-Nähe ein semantischer Parameter von Konstruktionen (Abschnitt 5.1). Sie entscheidet ebenfalls darüber, wie der Konstruktions-Frame für eine Konstruktion evoziert werden kann.

Die drei untersuchten Konstruktionen unterscheiden sich in ihrer formalen Abstraktheit dahingehend, dass die reflexive Weg-Konstruktion als spezifischste dieser drei Konstruktionen betrachtet werden kann, während die reflexive Bewegungskonstruktion als abstrakteste Konstruktion anzusehen ist und die reflexive Partikelverbkonstruktion in der Mitte liegt (vgl. Unterabschnitt 5.1.2). So besteht die reflexive Bewegungskonstruktion, wie in dem Beispielkonstrukt in (7) zu sehen, aus vier Strukturelementen (BEWEGENDES, EREIGNIS, KEE und WEG), von denen eines (das KEE) lexikalisch spezifiziert ist.

(7) \{[ Bewegendes Joas] [EReIgnis $_{\text {zwängte] [KEE }}$ sich] [Weg durch die Tür]\}, kaum hatte Liron sie einen Spalt weit geöffnet. (Boie, Kirsten: Skogland, Ort: Hamburg 2005, S. 147)

Die reflexive Partikelverbkonstruktion besteht, wie in (8-a) zu sehen, aus fünf Strukturelementen (BEwEGENDES, EREIGNIS, RichtUng, 〈WEG〉 und KEE), wobei der Status des KE 〈WEG) als Nicht-Kern-KE, das, wie in (8-b) zu sehen, nicht obligatorisch instanziiert werden muss, an dieser Zählung nichts ändert. Lexikalisch spezifiziert sind hier RICHTUNG und KEE.

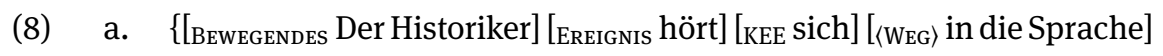
[RIchtung ein], in der sich eine Klasse von Menschen zu orientieren versucht\}, er tritt in den Zeithorizont ihrer Voraussetzungen und Erwartungen ein und hat, so an den Begrenzungen teil, denen sie selbst ausgesetzt war. (Schlögel, Karl: Petersburg, München Wien: Carl Hanser Verlag 2002, S. 90)

b. $\left\{\right.$ BBewegendes $_{\text {Er] [EReignis }}$ fragte] [KEe sich] [Richtung durch]\}. (Kopetzky, Steffen: Grand Tour, Frankfurt am Main: Eichborn 2002, S. 457) 
Die reflexive Weg-Konstruktion kommt wie die reflexive Partikelverbkonstruktion auf fünf Strukturelemente (BEWEgEndes, EREIGNIS, 〈WEG〉, KEE und KorE), vgl. das Konstrukt in (9). Das KE 〈WEG〉 ist wie bei der reflexiven Partikelverbkonstruktion als Nicht-Kern-KE einzustufen. Auch die reflexive Weg-Konstruktion besitzt zwei lexikalisch spezifizierte Strukturelemente - KEE und KorE -, wobei insbesondere Letzteres aber lexikalisch spezifizierter als die beiden KEE der reflexiven Partikelverbkonstruktion ist (vgl. Unterabschnitt 3.3.2).

(9) \{[Bewegendes Mein Schälmesser mit der dünnen Klinge] [EREIGnis säbelt] [KeE sich] [KoRE einen Weg] [〈WEG $\rangle$ durch die buschigen Petersilienköpfe]\}, während ich überlege, ob es tatsächlich Köpfe oder doch Blätter oder gar Büschel heißt. (Riedel, Susanne: Eine Frau aus Amerika, Berlin: Berlin Verlag 2003, S. 106)

Die sich daraus ergebende Hierarchie in der formalen Abstraktheit der drei Konstruktionen wird, wie in Abschnitt 5.1 erläutert, durch die Kombination zweier Dimensionen ermittelt: der Anzahl der Strukturelemente sowie der lexikalischen Spezifiziertheit. Bei der Frage nach möglichen Mechanismen für die Evokation des Konstruktions-Frames spielt nun insbesondere das Kriterium der lexikalischen Spezifiziertheit (Unterabschnitt 5.1.2) eine Rolle. Es unterscheidet die Strukturelemente einer Konstruktion danach, ob es sich dabei um KE, KEE oder KorE handelt. Welchem dieser drei Typen ein Strukturelement einer Konstruktion angehört, kann entscheidend bei der Frage sein, ob der Konstruktions-Frame durch (eine Instanz) dieses Strukturelements evoziert werden kann.

Eine wesentliche Eigenschaft eines Konstruktions-Frames, wie ich sie für die drei untersuchten Konstruktionen definiert habe, ist, dass der KonstruktionsFrame über die Konstrukte einer Konstruktion tendenziell invariant bleibt, was ihn am deutlichsten von lexikalischen Frames unterscheidet (vgl. Unterabschnitte 4.2.3 und 4.3.2). Bei der Frage, wie der Konstruktions-Frame evoziert werden kann und ob dies durch ein Strukturelement bzw. dessen Instanz geschehen kann, ist diese Invarianz zu berücksichtigten. So ist zu erwarten, dass lexikalisch invariante Strukturelemente tendenziell eher dazu geeignet sind, den KonstruktionsFrame ,lexikalisch` zu evozieren, und zwar unabhängig von der ebenso ,lexikalischen' Evokation des lexikalischen Frames. Mit ,lexikalischer' Evokation ist im Falle einer Evokation des Konstruktions-Frames durch ein lexikalisch invariantes Strukturelement also nicht dessen Evokation durch ein KtE des KE, das der Evokation des lexikalischen Frames vorbehalten ist, gemeint, sondern genau der umgekehrte Fall, dass er in einem davon unterschiedenen Strukturelement evoziert wird. Bei genauerem Hinsehen bestätigt sich diese Annahme für die drei untersuchten Konstruktionen - zumindest teilweise. 
- $\quad$ Ein KE wie EREIGNIS, das eine größere Varianz möglicher KtE (als LE sämtlicher möglicher lexikalischer Frames) aufweist, ist nur in einem einzigen Fall für die Evokation des Konstruktions-Frames relevant, nämlich dann, wenn der lexikalische Frame mit diesem identisch ist (vgl. Unterabschnitt 8.1.1). In allen anderen Fällen findet keine direkte ,lexikalische“ Evokation des Konstruktions-Frames statt (vgl. Unterabschnitt 8.2.2 sowie Abschnitt 8.3).

- $\quad$ Ein KEE wie das KEE der drei untersuchten Konstruktionen oder RICHTUNG der reflexiven Partikelverbkonstruktion kommt durch seine (für die drei untersuchten Konstruktionen) zwar nicht vollständige Invarianz (vgl. exemplarisch die Unterabschnitte 3.1.2 und 3.2.2), zumindest aber durch deutlich stärkere Beschränkungen als diejenigen des KE EREIGNIS (vgl. Unterabschnitt 5.3.2) hypothetisch betrachtet eher für die ,lexikalische‘ Evokation des Konstruktions-Frames infrage. Für die drei untersuchten Konstruktionen ist dies dennoch nicht der Fall, da nicht behauptet werden kann, dass ein Reflexivum oder eine der einschlägigen Verbpartikeln einen Frame wie Motion evoziert (vgl. Unterabschnitt 8.3.1).

- Das KorE der reflexiven Weg-Konstruktion hingegen ist prädestiniert für die ,lexikalische‘ Evokation des Konstruktions-Frames. Dies liegt in erster Linie daran, dass es ein Nomen beinhaltet, das als LE des Konstruktions-Frames Motion bzw. eines zu ihm in Frame-Nähe stehenden relatierten Frames (nämlich Self_motion) gelten kann (vgl. Unterabschnitt 8.3.2).

Für die drei untersuchten Konstruktionen lässt sich aus diesen Beobachtungen eine zumindest tendenzielle Korrelation zwischen formaler Abstraktheit einer Konstruktion und der Evokation des Konstruktions-Frames ableiten: Je formal abstrakter die Konstruktion ist, desto weniger Möglichkeiten zur ,lexikalischen‘ Evokation des Konstruktions-Frames bestehen. Umgekehrt gilt, dass, je formal spezifischer die Konstruktion ist, desto mehr Möglichkeiten zur ,lexikalischen` Evokation des Konstruktions-Frames bestehen. Diese Korrelation liegt quer zu dem Kontinuum der Evokation des Konstruktions-Frames, das in Abbildung 8.1 (Unterabschnitt 8.1.1) dargestellt ist. Während sich dies vor allem auf den Zusammenhang zwischen lexikalischem Frame und Konstruktions-Frame bezieht, kann es bei einer Konstruktion wie der reflexiven Weg-Konstruktion dazu kommen, dass der lexikalische Frame, auch wenn er mit dem Konstruktions-Frame identisch ist, wie üblich über ein KtE des KE EREIGNIS evoziert wird, während der KonstruktionsFrame gleichzeitig über ein davon unterschiedenes Strukturelement evoziert wird (vgl. Unterabschnitt 8.3.2). Die mit der Abstraktheit der drei untersuchten Konstruktionen tendenziell korrelierte Wahrscheinlichkeit der ,lexikalischen` Evokati- 
on des Konstruktions-Frames ist in Abbildung 8.2 illustriert, die eine Erweiterung von Abbildung 5.2 aus Unterabschnitt 5.1.2 darstellt.

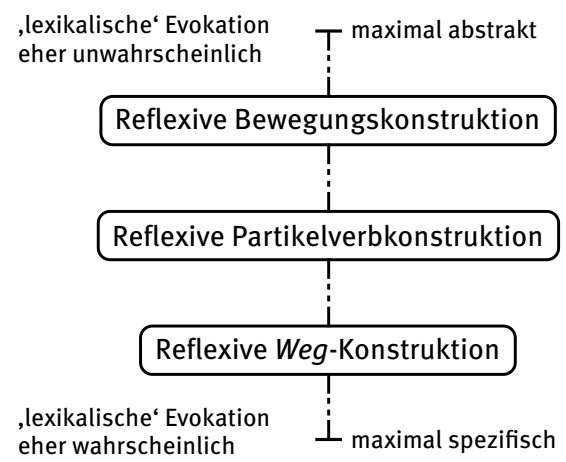

Abb. 8.2: Korrelation der Wahrscheinlichkeit der ,lexikalischen` Evokation des KonstruktionsFrames mit der formalen Abstraktheit der drei Konstruktionen

Als Zwischenfazit ist festzuhalten, dass neben der Frame-Nähe auch die formale Abstraktheit einer Konstruktion einen $\mathrm{zu}$ berücksichtigenden Einflussfaktor für die Mechanismen der Evokation des Konstruktions-Frames darstellt. Wie sich diese beiden Einflussfaktoren zueinander verhalten, möchte ich in der folgenden Diskussion klären.

\subsection{Evokation des Konstruktions-Frames bei relatierten lexikalischen Frames}

Unter den Einflussfaktoren, die die Evokation des Konstruktions-Frames steuern, kann die Frame-Nähe lexikalischer Frames (Unterabschnitt 8.1.2) als übergreifend bei der Differenzierung unterschiedlicher Mechanismen angesehen werden. Die formale Abstraktheit einer Konstruktion als Einflussfaktor (Unterabschnitt 8.1.3) ist ihr in dieser Hinsicht untergeordnet. Dies ist daran zu erkennen, dass die Frame-Nähe lexikalischer Frames wesentlich darüber bestimmt, ob weitere Einflussfaktoren bei der Evokation des Konstruktions-Frames eine Rolle spielen, denn insbesondere bei relatierten lexikalischen Frames treten, wie ich in diesem Abschnitt zeigen möchte, Einflussfaktoren wie die formale Abstraktheit der Konstruktion in den Hintergrund.

Aus diesem Grund trenne ich die Mechanismen der Evokation des Konstruktions-Frames, die ich in diesem und dem folgenden Abschnitt 8.3 diskutieren 
Tab. 8.1: Mechanismen der Evokation eines Konstruktions-Frames nach relatierten und unrelatierten lexikalischen Frames

\begin{tabular}{ll}
\hline Relatierte lexikalische Frames & Unrelatierte lexikalische Frames \\
\hline Identität von lexikalischem Frame und & Evokation durch ein KEE (Unterabschnitt 8.3.1) \\
Konstruktions-Frame (Unterabschnitt 8.2.1) & \\
Frame-Nähe und Spreading Activation (Unter- & Evokation durch ein KorE (Unterabschnitt \\
abschnitt 8.2.2) & $\begin{array}{l}8.3 .2) \\
\\
\end{array}$ \\
& Evokation durch syntagmatische Kombination \\
& von KtE und KEE (Unterabschnitt 8.3.3) \\
\hline
\end{tabular}

möchte, nach der Frame-Nähe lexikalischer Frames, also nach relatierten und unrelatierten Frames. Die insgesamt fünf Mechanismen, deren Diskussion die reflexive Bewegungskonstruktion, die reflexive Partikelverbkonstruktion und die reflexive Weg-Konstruktion nahelegen, können jeweils einer dieser beiden Kategorien lexikalischer Frames zugeordnet werden. Diese Zuordnung ist in Tabelle 8.1 dargestellt.

In diesem Abschnitt soll es zunächst um diejenigen Mechanismen gehen, die relatierte lexikalische Frames betreffen. Die Kategorie der relatierten lexikalischen Frames lässt sich, wie bereits in den Unterabschnitten 5.4.2 und 7.5.1 angesprochen, wiederum in zwei Unterkategorien aufteilen:

a) denjenigen lexikalischen Frame, der mit dem Konstruktions-Frame identisch ist;

b) diejenigen lexikalischen Frames, die zum Konstruktions-Frame in einer Frame-Nähe von +1 oder niedriger bzw. -1 oder niedriger stehen (relatierte lexikalische Frames im engeren Sinne).

Entsprechend dieser zwei Unterkategorien relatierter lexikalischer Frames lassen sich zwei Mechanismen der Evokation eines Konstruktions-Frames unterscheiden, die in Konstrukten, deren Konstrukt-Frames aus jenen lexikalischen Frames bestehen, zum Tragen kommen. Es handelt sich einerseits um den Fall, dass der lexikalische Frame mit dem Konstruktions-Frame identisch ist und somit direkt durch diesen lexikalischen Frame evoziert wird, und andererseits um die Fälle, in denen ausgehend von einem relatierten lexikalischen Frame mit einer Frame-Nähe von +1 oder niedriger bzw. -1 oder niedriger der Konstruktions-Frame aufgrund eines Spreading-Activation-Prozesses bei der Evokation eines entsprechenden lexikalischen Frames gewissermaßen ,mitevoziert' wird. Ersteren Fall mache ich im Folgenden zu Gegenstand von Unterabschnitt 8.2.1, während ich auf letzteren Fall in Unterabschnitt 8.2.2 eingehe. 


\subsubsection{Identität von lexikalischem Frame und Konstruktions-Frame}

$\mathrm{Zu}$ den relatierten lexikalischen Frames einer Konstruktion zählen nicht nur diejenigen Frames, die in einer Frame-Nähe von +1 oder niedriger (und ebenso -1 oder niedriger) zum Konstruktions-Frame stehen, sondern gleichsam derjenige Frame mit einer Frame-Nähe von 0, mit anderen Worten: der Konstruktions-Frame selbst. Wie bereits in Unterabschnitt 5.4.2 argumentiert, kann der Konstruktions-Frame, im Falle der drei untersuchten Konstruktionen also Motion, wenn er als lexikalischer Frame auftritt, als prototypischster aller lexikalischen Frames gelten. Dass Motion tatsächlich als lexikalischer Frame auftreten kann, habe ich für die reflexive Bewegungskonstruktion in Unterabschnitt 6.2.1 ausführlich gezeigt. Die hohe Prototypikalität dieses Frames spiegelt sich in der semantischen Motivierung der KtE wider: Motion ist unter den relatierten lexikalischen Frames, die allesamt in der Lage sind, alle KtE eines Konstrukts ohne einen zusätzlichen KonstruktionsFrame zu motivieren, der Prototyp. Nicht zuletzt aus diesem Grund kann sein FE Motion.THEME etwa für die Benennung des KE BEwEGENDEs herangezogen werden (Unterabschnitt 7.3.2).

Wie Tabelle 8.2 zeigt, ist die Identität zwischen lexikalischem Frame und Konstruktions-Frame für alle drei Konstruktionen, insbesondere für die reflexive Bewegungskonstruktion (RBKxn) und die reflexive Partikelverbkonstruktion (RPVKxn), durchaus zahlreich belegt.

Tab. 8.2: Konstrukte der drei Konstruktionen mit Identität von lexikalischem Frame und Konstruktions-Frame

\begin{tabular}{lrrr}
\hline Konstruktion & Konstrukte gesamt & Konstrukte mit Motion & Anteil \\
\hline RBKxn & 1.011 & 105 & $10,39 \%$ \\
RPVKxn & 850 & 24 & $2,82 \%$ \\
Weg-Kxn & 27 & 26 & $96,30 \%$ \\
\hline
\end{tabular}

Prototypisch beim Auftreten von Motion als lexikalischem Frame ist nun nicht nur die semantische Motivierung der KtE und damit die Konstitution des KonstruktFrames, sondern letztendlich auch die Evokation des Konstruktions-Frames. Die Identität zwischen lexikalischem Frame und Konstruktions-Frame stellt den einfachsten Fall der ,lexikalischen' Evokation des Konstruktions-Frames dar. Der Konstruktions-Frame wird hier direkt in Gestalt des lexikalischen Frames evoziert, und zwar durch ein KtE des KE EREIGNIS, das unmittelbar eine LE des Konstruktions-Frames darstellt. 
Einige Beispiele für die drei untersuchten Konstruktionen sollen dies noch einmal illustrieren. Für die reflexive Bewegungskonstruktion zeigen die Belege in (10)-(16) Konstrukte, in denen lexikalischer Frame und Konstruktions-Frame identisch sind. Die KtE bewegen (move.v), begeben (go.v), schlängeln (snake.v), winden (wind.v), mäandern (meander.v), verschieben und rücken (beide move.v) evozieren Motion als lexikalischen Frame, der direkt dem Konstruktions-Frame entspricht. Sie evozieren somit zugleich den Konstruktions-Frame.

(10) a. Die deutsche Seele ist bewegt, [Motion bewegt] sich durch Berlin und findet sich nicht wieder. (Die Zeit, 17.02.2000, Nr. 8)

b. Die augenblickliche Wirtschaftslage [Motion bewege] sich in Richtung Armut, was das Resultat der ausbeuterischen Privatisierung sei. (Archiv der Gegenwart, 2001 [2000])

c. Eine Ameise scheint sich auf der Schnur nur von links nach rechts [Motion bewegen] zu können.“ (Die Zeit, 10.02.2000, Nr. 7)

(11) a. Auch ich [Motion begebe] mich in den Garten und pflücke mir ein bescheidenes Sträußchen kriechender Kapuzinerkresse. (Noll, Ingrid: Ladylike, Zürich: Diogenes 2006, S. 137)

b. Zu bestimmten Zeiten, wenn die Buschtrommel des HSV ins Volksparkstadion oder die des Ersten FC Kaiserslautern auf den Betzenberg ruft, verlassen die Fans ihre Lehmhütten, [Motion begeben] sich zu den Versammlungsplätzen der Männer, nehmen berauschende Getränke oder Drogen ein, schmücken sich mit den Totems des Stammes und tragen auf ihrer Haut die Farben der Kriegsbemalung auf. (Schwanitz, Dietrich: Männer, Frankfurt a. M.: Eichborn 2001, S. 40)

c. Dr. Hans Wilhelm Stein, Burgherr von Saaleck, verbarg die beiden und [Motion begab] sich nach München, in Ehrhardts Hauptquartier, um falsche Pässe und einen Fluchtwagen zu organisieren. (Die Zeit, 30.03.2000, Nr. 14)

(12) a. Ich [Motion schlängelte] mich durch die Autos und die Abgase, die rot zwischen den Schlußlichtern dampften, und entdeckte gerade noch rechtzeitig die kräftige Gestalt von John Bird hinter dem erleuchteten Schaufenster. (Franck, Julia: Lagerfeuer, Köln: DuMont Literatur und Kunst Verlag 2003, S. 256)

b. Die Luft war stickig, der Rauch [Motion schlängelte] sich über die Brocken, kaum vom Kohlenstaub unterscheidbar. (Schrott, Raoul: Tristan da Cunha oder die Hälfte der Erde; Hanser Verlag 2003, S. 435) 
(13) Jonas [Motion wand] sich aus dem Kofferraum nach vorne auf die Rückbank des Autos. (Glavinic, Thomas: Die Arbeit der Nacht, München Wien: Carl Hanser Verlag 2006, S. 346)

(14) So [Motion mäandern] sich die nackten und verschlungenen Leiber durch die Werbung und über die Titel jener der Werbeästhetik folgenden Magazine. (Die Zeit, 13.01.2000, Nr. 3)

(15) Der Schwerpunkt des Westens [Motion verschob] sich in die USA. (Die Zeit, 02.03.2000, Nr. 10)

(16) Er [Motion rückt] sich in den Mittelpunkt. (Schwanitz, Dietrich: Männer, Frankfurt a. M.: Eichborn 2001, S. 73)

Analoge Beobachtungen lassen sich für die reflexive Partikelverbkonstruktion machen. Wie die Daten in Tabelle 8.2 zeigen, liegt die Frequenz von Konstrukten mit Motion als lexikalischem Frame zwar unter derjenigen der reflexiven Bewegungskonstruktion, dennoch finden sich zahlreiche Konstrukte, in denen eine Identität von lexikalischem Frame und Konstruktions-Frame besteht. Die Belege in (17)(19) zeigen Beispiele für die ebenso für die reflexive Bewegungskonstruktion belegten LE bewegen (move.v), schlängeln (snake.v) und winden (wind.v), die direkt Motion als lexikalischen Frame und somit Konstruktions-Frame evozieren.

(17) a. Bei jedem Zweig, der unter ihren Schuhen zerbrach, schrak Meggie zusammen, doch zum Glück machten auch Basta und Flachnase einigen Lärm, während sie sich durch das Dickicht den Berg hinab[Motion bewegten]. (Funke, Cornelia: Tintenherz, Hamburg: Cecilie Dressler Verlag 2003, S. 230)

b. Er könne sich zu wirklichem Frieden fort [Motion bewegen] oder ins Chaos zurückfallen. (Archiv der Gegenwart, 2001 [2000])

c. Im Grunde praktizierte und propagierte Erhard wirtschaftspolitische Vorstellungen, auf die sich Adenauer selbst zunehmend hin[Motion bewegte], seitdem er im Frühjahr 1946 an die Spitze der CDU in der britischen Zone gelangt war. (Schwarz, Hans-Peter: Anmerkungen zu Adenauer, München: Deutsche Verlags-Anstalt 2005 [2004], S. 53)

(18) a. Die Karkasse war an ihrer Unterseite aufgerissen, die Eingeweide waren hervorgequollen, [Motion schlängelten] sich auf und dümpelten im Wasser zwischen den Algen und dem Kelp. (Schrott, Raoul: Tristan da Cunha oder die Hälfte der Erde; Hanser Verlag 2003, S. 72)

b. „Niemand da?“ fragte der Pförtner, als Christian sich an den Gummibäumen vorbei[Motion schlängelte]. (Düffel, John von: Houwelandt, Köln: DuMont Literatur und Kunst Verlag 2004, S. 212) 
c. Ellen jedoch wand und [Motion schlängelte] sich zwischen den Leuten hindurch, man machte ihr Platz wie einer Kellnerin. (Schulze, Ingo: Neue Leben, Berlin: Berlin Verlag 2005, S. 440)

(19) a. Der dritte und letzte Fahrgast, der sich heraus[Motion windet], ist zu unserer grenzenlosen Verblüffung niemand anderer als der lange, leichenblasse Rudi. (Noll, Ingrid: Ladylike, Zürich: Diogenes 2006, S. 230)

b. Mit jedem Schritt war er der Biegung des Flusses näher gekommen und hatte weiter stromaufwärts gesehen, zum Eibsandsteingebirge, zum Lilienstein und zum Königstein, zwischen denen sich die Elbe hindurch[Motion wand], darüber blaßblaue Wolken, deren Ränder sich dunkel vor dem gelblichweißen Licht abgehoben hatten. (Schulze, Ingo: Neue Leben, Berlin: Berlin Verlag 2005, S. 714)

c. Seine Scheunenvierteluhr war entworfen, und eine Raupe aus grauen, Gestalten [Motion wand] sich bei Sonnenuntergang, schlurfend, schweigend, am Schloßberg hoch. (Koneffke, Jan: Paul Schatz im Uhrenkasten, Köln: DuMont Buchverlag 2000, S. 228)

Hinsichtlich der LE, die Motion als lexikalischen Frame und Konstruktions-Frame zugleich evozieren, stellt die reflexive Weg-Konstruktion, wie bereits in Unterabschnitt 3.3.2 angesprochen, eine Besonderheit dar, da sich für das Verb bahnen, das in 26 von insgesamt 27 Konstrukten dieser Konstruktion erscheint, keine direkte Entsprechung in FrameNet findet. Ein einfacher Abgleich mit Wörterbuchdaten lässt es aber plausibel erscheinen, diesem Verb zumindest heuristisch die Eigenschaft zuzuschreiben, Motion als lexikalischen Frame zu evozieren. Im DWDS findet sich im Eintrag zum Verb bahnen als erstes und einziges Verwendungsmuster die Angabe „sich, jmdm. einen Weg bahnen“.13 Unter den Verwendungsbeispielen finden sich sodann zahlreiche Konstrukte der reflexiven Weg-Konstruktion. ${ }^{14} \mathrm{Da}$ ich davon ausgehe, dass der Konstruktions-Frame dieser Konstruktion, wie derjenige der anderen beiden untersuchten Konstruktionen, Motion ist, erscheint die heuristische Annahme von bahnen als LE von Motion gerechtfertigt. Die drei Bele-

13 https://www.dwds.de/wb/bahnen (zuletzt abgerufen am 07.09.2021).

14 Wie Verhagen (2003a: 333, 2003b: 35-36) für das Niederländische feststellt, ist die lexikalische Bedeutung von bahnen kaum zu exemplifizieren, ohne dieses Verb in der reflexiven WegKonstruktion zu gebrauchen. Dies ist ein starkes Anzeichen dafür, dass der Konstruktions-Frame mit dem lexikalischen Frame, den diese LE evoziert, identisch sein muss, denn, so resümiert Verhagen (2002: 411) für das niederländische banen: „If one tries to describe its meaning, then one essentially ends up with something very similar to the meaning of the entire construction." Vgl. auch Verhagen (2003a: 333, 2003b: 36). 
ge in (20) sollen entsprechende Konstrukte der reflexiven Weg-Konstruktion noch einmal illustrieren.

(20) a. Jawohl, denn die Damen wirken „schwerfällig und lustlos“, [Motion bahnen] sich an Bord nur mühsam ihren Weg durch die engen Gänge und ecken sehr wahrscheinlich auch bei solchen Passagieren an, die es in höchstem Maße unerquicklich finden, ihren Anisschnaps von einem Fesselballon serviert zu bekommen. (Die Zeit, 10.02.2000, Nr. 7)

b. Ich beobachtete, wie Doreen die Hände des Pfarrers und seiner Frau abwehrte, sie ließ die beiden zurück und [Motion bahnte] sich einen Weg zu den Essenswagen. (Franck, Julia: Lagerfeuer, Köln: DuMont Literatur und Kunst Verlag 2003, S. 301)

c. Was passiert ist, ist passiert“, und er drehte sich um und [Motion bahnte] sich langsam durch die Büsche seinen Weg zurück zum Gutshaus. (Boie, Kirsten: Skogland, Ort: Hamburg 2005, S. 185)

Der Fall einer Identität von lexikalischem Frame und Konstruktions-Frame ist somit für alle drei untersuchten Konstruktionen belegt. Für diese Konstruktionen kann also davon ausgegangen werden, dass in der jeweiligen Menge von Konstrukten, auf die dieser Fall zutrifft, der Konstruktions-Frame direkt durch ein KtE des KE EREIGNIS evoziert wird, da dieses KtE eben als LE von Motion zu verstehen ist. Tabelle 8.2 zeigt aber zugleich, dass die Identität zwischen lexikalischem Frame und Konstruktions-Frame zwar durchaus zahlreich belegt ist, zumindest für die reflexive Bewegungskonstruktion und die reflexive Partikelverbkonstruktion aber nur einen kleinen Teil ihrer jeweiligen Konstrukte ausmacht. Für alle übrigen Konstrukte muss es andere Mechnismen der Evokation des Konstruktions-Frames geben. Bleibt man zunächst noch bei relatierten lexikalischen Frames, führt ausgehend vom Konstruktions-Frame der Weg zunächst zu allen anderen zu ihm relatierten lexikalischen Frames, die in einer Frame-Nähe von +1 oder niedriger bzw. -1 oder niedriger $\mathrm{zu}$ ihm stehen.

\subsubsection{Frame-Nähe und Spreading Activation}

Die Konstrukte, in denen der lexikalische Frame mit dem Konstruktions-Frame identisch ist, können als direkteste ,lexikalische“ Evokation des KonstruktionsFrames verstanden werden. Sie stellen allerdings nicht die einzigen Fälle dar, in denen der Konstruktions-Frame über ein KtE des KE EREIGNIS evoziert werden kann. 
Ausgehend von der im letzten Unterabschnitt 8.2.1 diskutierten Beobachtung, dass der Konstruktions-Frame am basalsten in solchen Konstrukten evoziert wird, in denen lexikalischer Frame und Konstruktions-Frame identisch sind, ist es nur ein kleiner Schritt, den Mechanismus der Evokation des Konstruktions-Frames durch KtE des KE EREIGNIS auf lexikalische Frames zu übertragen, die zwar nicht mit dem Konstruktions-Frame identisch sind, ihm aber dennoch nahekommen. Die Rede ist einmal mehr von allen relatierten Frames, die in einer Frame-Nähe von +1 oder niedriger bzw. -1 oder niedriger zum Konstruktions-Frame stehen. Sie bilden das in Unterabschnitt 5.4.3 vorgestellte System von Frames, die die FrameNähen von Motion definieren. Wenn also KtE des KE EREIGNIS, die LE von Motion darstellen, den Konstruktions-Frame evozieren können, können dies dann auch - gewissermaßen ,indirekt ‘ - KtE, die als LE relatierter Frames in Frame-Nähe zu Motion stehen? Ich möchte diese Frage positiv beantworten und in diesem Unterabschnitt zeigen, dass die LE aller relatierten lexikalischen Frames in der Lage sind, den Konstruktions-Frame zu evozieren, eben weil die entsprechenden lexikalischen Frames zu ihm in Frame-Nähe stehen. Stützen lässt sich diese Annahme unter Rückgriff auf ein netzwerktheoretisches Modell der Spreading Activation (vgl. überblickend Barsalou 1992a: 45-49; Traugott \& Trousdale 2013: 54-56; Matos et al. 2017; Diessel 2019: 94; Schmid 2020: 44-45), das, wie ich im Folgenden ausführen möchte, in Einklang mit der variierenden Prototypikalität relatierter lexikalischer Frames in Bezug auf den Konstruktions-Frame (Unterabschnitt 5.4.2) steht. $^{15}$

Die Grundidee der Spreading-Activation-Theorie, wie sie etwa von Collins \& Loftus (1975) oder Anderson (1983) vertreten wird, besteht darin, dass bei der Aktivierung eines Knotens in einem semantischen Netzwerk zugleich weitere Knoten, die zu diesem in Relation stehen, mitaktiviert werden, wobei die Aktivierung mit zunehmender Distanz vom Ausgangsknoten schwächer wird:

When a concept is processed (or stimulated), activation spreads out along the paths of the network in a decreasing gradient. The decrease is inversely proportional to the accessibility or strength of the links in the path. (Collins \& Loftus 1975: 411)

Frame-semantisch betrachtet ist ein solches semantisches Netzwerk nichts anderes als ein System von Frames und Relationen zwischen diesen (vgl. Czulo et al. 2019: 30). Konstruktionssemantisch gewendet dient als Ausgangsknoten der

15 Frame-semantische und konstruktikographische Anwendung findet die Spreading-ActivationTheorie etwa für die computerlinguistische Ermittlung der besten Interpretation einer Konstruktion (Matos et al. 2017) oder der Entwicklung einer frame-semantischen Metrik zur Evaluation maschineller Übersetzungen (Czulo et al. 2019). 
Konstruktions-Frame, das semantische Netzwerk ist mit dessen System an FrameNähen zu identifizieren, es besteht also aus dem Konstruktions-Frame sowie allen zu ihm relatierten lexikalischen Frames. Wird einer dieser Frames evoziert, so werden zugleich alle Frames, die zu diesem in einer Frame-zu-Frame-Relation stehen, mitevoziert, auch über mehrere Hierarchieebenen hinweg (also etwa auch für Frame-Nähen von +2 und +3 ). Die Evokation breitet sich also gewissermaßen ausgehend von einem lexikalischen Frame in das System der Frame-Nähen aus. Barsalou (1992a: 47) weist darauf hin, dass Spreading-Activation-Prozesse gleichermaßen ,bottom up' wie auch ,top down' verlaufen können. Das heißt, dass der direkt evozierte Frame sowohl auf einer niedrigeren als auch auf einer höheren Hierarchieebene liegen kann - in beiden Fällen werden weitere Frames, die zu ihm in Frame-zu-Frame-Relation stehen, mitevoziert, sowohl ,nach unten` als auch ,nach oben'.

Ein simples Beispiel: Wird in einem Konstrukt der reflexiven Bewegungskonstruktion wie (21) der lexikalische Frame Self_motion evoziert, der zum Konstruktions-Frame Motion in einer Frame-Nähe von +1 steht, so wird zugleich Motion mitevoziert. Wird ein zum Konstruktions-Frame relatierter lexikalischer Frame mit einer Frame-Nähe von +1 oder niedriger bzw. -1 oder niedriger evoziert, wird der Konstruktions-Frame gleichzeitig mitevoziert.

(21) Ich [self_motion schlich] mich vorsichtig an ihm vorbei zu dem Gewürzregal, ließ ihn aber nicht aus den Augen. (Düffel, John von: Vom Wasser, München: dtv 2006, S. 260)

Nun lässt sich allerdings nicht nur die Annahme der reinen Mitevokation des Konstruktions-Frames bei der Evokation eines relatierten lexikalischen Frames mit Hilfe der Spreading-Activation-Theorie stützen, auch die Annahme der abnehmenden Aktivierungsstärke mit zunehmender Distanz vom Ausgangsknoten - also dem Konstruktions-Frame - lässt sich auf das Konzept der Frame-Nähe übertragen. Mit abnehmender Frame-Nähe eines relatierten lexikalischen Frames zum Konstruktions-Frame sinkt die Stärke der Evokation des Letzteren. Diese Idee steht im Einklang mit der Annahme der sinkenden Prototypikalität eines relatierten lexikalischen Frames in niedrigerer Frame-Nähe zum Konstruktions-Frame (Unterabschitt 5.4.2). Diese abnehmende Prototypikalität eines relatierten lexikalischen Frames mit abnehmender Frame-Nähe lässt sich mit der schwächeren Aktivierungsstärke dieses lexikalischen Frames aus Sicht des Konstruktions-Frames begründen. Mit anderen Worten: Je weniger prototypisch ein relatierter lexikalischer Frame ist, desto weniger stark ist bei seiner Evokation die gleichzeitige Evokation des Konstruktions-Frames. Mit abnehmender Frame-Nähe eines relatierten 
lexikalischen Frames sinkt nicht nur die Mitevokation des Konstruktions-Frame, auch die semantische Ähnlichkeit des Ersteren zum Letzteren nimmt ab:

The conceptual (semantic) network is organized along the lines of semantic similarity. The more properties two concepts have in common, the more links there are between the two nodes via these properties and the more closely related are the concepts. (Collins \& Loftus 1975: 411)

Wenn Barsalou (1992a: 47) darauf hinweist, dass Spreading Activation gleichermaßen ,bottom up' wie auch ,top down' verlaufen kann, entspricht Ersteres einer positiven Frame-Nähe, für die das Konstrukt in (21) mit Self_motion ein Beispiel ist, während Letzteres einer negativen Frame-Nähe entspricht. Ein Beispiel dafür ist das Konstrukt der reflexiven Bewegungskonstruktion in (22) mit dem lexikalischen Frame Cause_motion, der zu Motion in einer Frame-Nähe von -1 steht, diesem also übergeordnet ist.

(22) Er [Cause_motion schob] sich ächzend nach vorn auf die Kante seines Sessels, erhob sich aber dann trotz seines mächtigen Bauches überraschend mühelos. (Schulze, Ingo: Neue Leben, Berlin: Berlin Verlag 2005, S. 25)

An zwei Beispielkomplexen möchte ich den Mechanismus der Evokation des Konstruktions-Frames mittels Spreading Activation etwas weiter ausführen. Dafür seien zwei Typen von Frame-zu-Frame-Relationen in den Blick genommen: die Vererbungsrelation und die Benutzt-Relation. Sie eignen sich besonders zur Illustration von Spreading-Activation-Prozessen bei der Evokation des KonstruktionsFrames, weil für sie zahlreiche Konstrukte der reflexiven Bewegungskonstruktion und der reflexiven Partikelverbkonstruktion belegt sind und die Benutzt-Relation zudem über Frames bis zu einer Frame-Nähe von +3 zu Motion verfügt, während die Vererbungsrelation bis zu einer Frame-Nähe von +2 reicht (vgl. die Tabellen 5.12 und 5.13 in Unterabschnitt 5.4.3).

In Abbildung 8.3 ist ein Ausschnitt der zu Motion in Vererbungsrelation stehenden lexikalischen Frames dargestellt. Für diese Relation sind Frame-Nähen von bis zu +2 in FrameNet dokumentiert, für die Frame-Nähe +1 sei hier exemplarisch einmal mehr der lexikalische Frame Self_motion genannt, für die FrameNähe +2 der lexikalische Frame Fleeing.

Der lexikalische Frame Self_motion ist sowohl für die reflexive Bewegungskonstruktion als auch für die reflexive Partikelverbkonstruktion belegt, während Fleeing ausschließlich für die reflexive Bewegungskonstruktion belegt ist. Unter (23) sind noch einmal einige Beispielkonstrukte für Self_motion und die reflexive Bewegungskonstruktion, unter (24) für Self_motion und die reflexive Partikelverbkonstruktion zusammengestellt. 
$+1$

$+2$
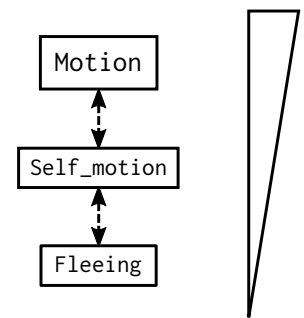

Abb. 8.3: Ausschnitt der zum Konstruktions-Frame Motion in Vererbungsrelation relatierten Frames in FrameNet 1.7 mit ihrer Aktivierungsstärke

a. Wieder einmal [self_motion schleicht] sich Wenders vom Himmel aus an seine Geschichte heran, gewissermaßen engelsgleich. (Die Zeit, 03.02.2000, Nr. 6)

b. General Motors geht es mit seiner Tochter Opel kaum besser, Fiat [self_motion robbt] sich mühsam aus den roten Zahlen, und Renault hat die Übernahme von Nissan noch längst nicht bewältigt. (Die Zeit, 24.02.2000, Nr. 9)

c. Die Sonne sinkt, die Campesinos auf dem Platz [self_motion verkriechen] sich in ihre Decken. (Die Zeit, 05.01.2000, Nr. 2)

(24) a. Überall schien sie zu sein, ein bösartiges, körperloses Wesen, das nur darauf gewartet hatte, dass die Glühbirne verlosch, und sich nun in der Finsternis an sie heran[self_motion schlich], um sie in ihre kalten Arme zu nehmen. (Funke, Cornelia: Tintenherz, Hamburg: Cecilie Dressler Verlag 2003, S. 169)

b. Dort, wo sich die Brandung nicht wundwusch an dem rötlichen, von der Sonne gehärteten Stein, [self_motion rankten] sich Muscheln die Felswände hinauf. (Düffel, John von: Houwelandt, Köln: DuMont Literatur und Kunst Verlag 2004, S. 24)

c. Ob er sich in der Manier Keith Jarretts in ein Thema hinein[self_motion schaukelt], es aufsplittert oder choralartig streng entwickelt, immer ist sein Formbewusstsein spürbar. (Die Zeit, 17.02.2000, Nr. 8)

Der lexikalische Frame Self_motion ist dem Konstruktions-Frame Motion direkt untergeordnet, weshalb er die Frame-Nähe von +1 erhält. Die Aktivierungsstärke, mit der LE wie schleichen (sneak.v), robben, verkriechen (beide crawl.v) ranken (climb.v) oder schaukeln (swing.v) mitevozieren, kann also als durchaus hoch eingeschätzt werden. Die Ähnlichkeit zwischen beiden Frames ist schon am gemeinsamen Namensbestandteil motion zu erkennen (vgl. auch zu Gemeinsamkei- 
ten ihrer FE die Unterabschnitte 6.2.1 und 7.3.2). Konstrukte, in denen ein lexikalischer Frame wie Self_motion evoziert wird, der in einer Frame-Nähe von +1 zum Konstruktions-Frame steht, evozieren Letzteren also auf kürzestem Wege mit.

Einen längeren Weg zum Konstruktions-Frame bringt der lexikalische Frame Fleeing mit sich, der zu Motion in einer Frame-Nähe von +2 steht. In (25) finden sich zwei Beispielkonstrukte der reflexiven Bewegungskonstruktion für diesen Frame.

(25) a. Die [Fleeing flüchtet] sich in eine Affäre mit ihrem Maklervorbild, dem noch größeren Fassadenkünstler Buddy Kane (Peter Gallagher). (Die Zeit, 20.01.2000, Nr. 4)

b. Panik und Niedergeschlagenheit wechselten auch bei ihr ab, sie [Fleeing flüchtete] sich zu ihrem chirurgischen Besteck, in ihre SchädelHirn-Traumata. (Stadler, Arnold: Sehnsucht, Köln: DuMont Literatur und Kunst Verlag 2002, S. 37)

Durch die Frame-Nähe von +2 ist die Aktivierungsstärke, mit der der KonstruktionsFrame bei LE wie flüchten (flee.v) mitevoziert wird, schwächer als bei Self_motion. Dennoch kann auch hier davon ausgegangen werden, dass Motion bei allen Konstrukten, deren Konstrukt-Frames aus einem lexikalischen Frame wie Fleeing in Frame-Nähe von +2 besteht, mitevoziert wird. Die geringere Frame-Nähe zeigt also zugleich die geringere Aktivierungsstärke des Konstruktions-Frames an.

Ähnliche Beobachtungen lassen sich für die Benutzt-Relation aufzeigen. Der einzige Unterschied zur Vererbungsrelation besteht darin, dass die BenutztRelation Frame-Nähen zu Motion von bis zu +3 aufweist, während für die Vererbungsrelation lediglich Frame-Nähen von +2 belegt sind, $\mathrm{zu}$ denen der soeben betrachtete lexikalische Frame Fleeing gehört. Wie Abbildung 8.4 zeigt, zählt zu den Frames mit einer Frame-Nähe von +1 Body_movement, diesem wiederum ist Facial_expression untergeordnet, weshalb dieser Frame eine Frame-Nähe von +2 erhält. Facial_expression wiederum ist Making_faces untergeordnet, der dann in einer Frame-Nähe von +3 zu Motion steht. Weiter entferntere Frames sind für diese Relation und auch alle anderen Relationen, wie in Unterabschnitt 5.4.3 aufgezeigt, nicht belegt.

Der lexikalische Frame Body_movement ist wie Self_motion sowohl für die reflexive Bewegungskonstruktion als auch für die reflexive Partikelverbkonstruktion belegt. Unter (26) sind einige Beispielkonstrukte für die reflexive Bewegungskonstruktion zusammengestellt, während (27) Beispielkonstrukte für die reflexive Partikelverbkonstruktion zeigt.

(26) a. Und Paul bestrafte sie mit Großvaters eisernem Schweigen, mied Annas Keller, einen Tag, zwei Tage, und [Body_movement warf] sich an Hau- 


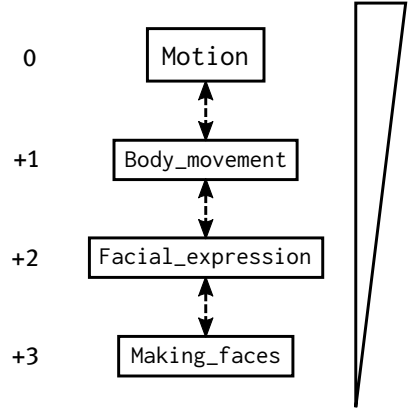

Abb. 8.4: Ausschnitt der zum Konstruktions-Frame Motion in Benutzt-Relation relatierten Frames in FrameNet 1.7 mit ihrer Aktivierungsstärke

eisens Klavier und stimmte Donnerakkorde an, um sie zu zerschmettern und Mores zu lehren. (Koneffke, Jan: Paul Schatz im Uhrenkasten, Köln: DuMont Buchverlag 2000, S. 108)

b. Er aber lief in sein Zimmer am Ende des Flurs und [Body_movement beugte] sich weit aus dem Fenster. (Kuckart, Judith: Lenas Liebe, Köln: DuMont Literatur und Kunst Verlag 2002, S. 224)

c. Er [Body_movement zitterte] sich in einen Dämmerschlaf, von dem er nicht wußte, ob er zehn Minuten dauerte oder drei Stunden. (Glavinic, Thomas: Die Arbeit der Nacht, München Wien: Carl Hanser Verlag 2006, S. 167)

(27) a. Er [Body_movement beugte] sich seitlich zu dem Hund hinab, ein Singsang, beruhigend, ja einlullend - auf englisch! (Schulze, Ingo: Neue Leben, Berlin: Berlin Verlag 2005, S. 83)

b. Zuckende, kauernde, kraxelnde Leiber auf Boden, Treppen und Geländern, eine makabre Arme-Leute-Prozession [Body_movement wälzt] sich voran, Salatköpfe rollen, in den Lüften ein wüstes Blasen und Dröhnen. (Die Zeit, 20.04.2000, Nr. 17)

c. Sie [Body_movement heben] sich über den Rest der Welt hinaus. (Riedel, Susanne: Eine Frau aus Amerika, Berlin: Berlin Verlag 2003, S. 222)

Analog zu Self_motion wird auch in den Fällen, in denen der Konstrukt-Frame aus Body_movement besteht, der Konstruktions-Frame durch LE wie werfen (throw.v), beugen (bend.v), zittern (shiver.v), wälzen (roll.v) oder heben (lift.v) mitevoziert. Die Frame-Nähe von +1 spricht auch bei der Benutzt-Relation für eine hohe Aktivierungsstärke des Konstruktions-Frames. 
Für den lexikalischen Frame Facial_expression, der in einer Frame-Nähe von +2 in der Benutzt-Relation zu Motion steht, sind für alle drei untersuchten Konstruktionen keine Konstrukte belegt. Eine sinkende Aktivierungsstärke mit abnehmender Frame-Nähe lässt sich für diese Frame-zu-Frame-Relation dennoch aufzeigen. Für den lexikalischen Frame Making_faces mit der Frame-Nähe +3 ist für die reflexive Bewegungskonstruktion ein Konstrukt belegt, vgl. (28).

(28) Ein polnisches Model (Goshia mit Namen) am Tisch gegenüber versucht sich seit 20 Minuten in unser Gespräch reinzu[Making_faces grinsen]. (Die Zeit, 30.03.2000, Nr. 14)

Die niedrige Frame-Nähe ist an diesem Konstrukt intuitiv erkennbar und entsprechend niedrig ist auch die Aktivierungsstärke, mit der Motion durch eine LE wie grinsen (grin.v) mitevoziert wird. Konstrukte mit solch niedrigen Frame-Nähen und entsprechend niedriger Prototypikalität markieren den Übergang zu unrelatierten lexikalischen Frames. Es scheint deshalb kein Zufall zu sein, dass niedrigere Frame-Nähen als +3 zumindest für Motion in FrameNet 1.7 nicht dokumentiert sind.

Die Aktivierungsstärke eines lexikalischen Frames auf den KonstruktionsFrame bezogen lässt sich nicht nur an der Frame-Nähe des Ersteren erkennen. Empirisch lässt sie sich durch die Token-Frequenz des entsprechenden lexikalischen Frames messen. Auf dieses Kriterium der Frequenz weist Anderson (1983) hin:

The strength of a node is a function of its frequency of exposure. The strength of the elements of focused units determines the amount of activation they can emit into the network. Also, more activation is sent down the paths leading to the stronger nodes. Thus, more activation will accumulate in those parts of the network that have stronger units. (Anderson 1983: 266)

Vor diesem Hintergrund ist erwartbar, dass Token-Frequenz und Frame-Nähe korreliert sind: Mit abnehmender Frame-Nähe sollte auch die Anzahl der Konstrukte, die für entsprechende lexikalische Frames belegt sind, sinken. Genau dies ist für die reflexive Bewegungskonstruktion und die reflexive Partikelverbkonstruktion der Fall. Die zwei Beispielkomplexe der Vererbungsrelation und der BenutztRelation sollen dies verdeutlichen. Wie aus Tabelle 8.3 für die Vererbungsrelation hervorgeht, nimmt die Anzahl der Konstrukte zwischen dem Frame Self_motion $(+1)$ und dem Frame Fleeing (+2) deutlich ab. Für die reflexive Partikelverbkonstruktion ist, wie erwähnt, Fleeing nicht belegt.

Ähnliches lässt sich für die Benutzt-Relation beobachten. In Tabelle 8.4 ist zu sehen, dass die Token-Frequenz zwischen Body_movement (+1) und Making_faces (+3) sehr deutlich sinkt, während für Facial_expression keine Konstrukte belegt 
Tab. 8.3: Token-Frequenzen lexikalischer Frames in Vererbungsrelation in FrameNet 1.7 mit Frame-Nähe zum Konstruktions-Frame Motion

\begin{tabular}{rlrrr}
\hline Frame-Nähe & Frame & RBKxn & RPVKxn & Weg-Kxn \\
\hline 0 & Motion & 105 & 24 & 26 \\
+1 & Self_motion & 159 & 56 & 0 \\
+2 & Fleeing & 5 & 0 & 0 \\
\hline
\end{tabular}

sind. Aus der vollständigen Dokumentation aller relatierten lexikalischen Frames, die sich in den Tabellen 5.12 bis 5.17 in Unterabschnitt 5.4.3 findet, ist jedoch abzulesen, dass in der Benutzt-Relation andere Frames mit einer Frame-Nähe von +2 durchaus belegt sind. Die dort dargestellten Daten bieten somit gleichermaßen einen Überblick über die Aktivierungsstärken der relatierten lexikalischen Frames, die insgesamt (wie etwa in Tabelle 5.18 in Unterabschnitt 5.4.3 abzulesen) deutlich mit der Frame-Nähe lexikalischer Frames korrelieren.

Tab. 8.4: Token-Frequenzen lexikalischer Frames in Benutzt-Relation in FrameNet 1.7 mit FrameNähe zum Konstruktions-Frame Motion

\begin{tabular}{rlrrr}
\hline Frame-Nähe & Frame & RBKxn & RPVKxn & Weg-Kxn \\
\hline 0 & Motion & 105 & 24 & 26 \\
+1 & Body_movement & 246 & 118 & 0 \\
+2 & Facial_expression & 0 & 0 & 0 \\
+3 & Making_faces & 1 & 0 & 0 \\
\hline
\end{tabular}

Die Evokation des Konstruktions-Frames mittels eines Spreading-ActivationProzesses über den lexikalischen Frame ist neben der direkten Evokation des Konstruktions-Frames bei der Identität von lexikalischem Frame und Konstruktions-Frame (Unterabschnitt 8.2.1) der zweite Mechanismus, der relatierte lexikalische Frames betrifft. Zusammengenommen decken diese beiden Mechanismen alle Konstrukte einer Konstruktion ab, deren Konstrukt-Frames aus relatierten lexikalischen Frames einschließlich des Konstruktions-Frames selbst bestehen (im Kontinuum der Evokation eines Konstruktions-Frames in Abbildung 8.1 in Unterabschnitt 8.1.1 links und in der Mitte). Bei unrelatierten lexikalischen Frames können diese Mechanismen allerdings nicht zum Einsatz kommen. 


\subsection{Evokation des Konstruktions-Frames bei unrelatierten lexikalischen Frames}

Mit den in Abschnitt 8.2 diskutierten Mechanismen der Evokation des Konstruktions-Frames bei relatierten Frames ist bereits eine große Menge der Konstrukte der drei untersuchten Konstruktionen abgedeckt (vgl. die Angaben in Tabelle 5.18 in Unterabschnitt 5.4.3). Für Konstrukte, deren Konstrukt-Frames unrelatierte lexikalische Frames beinhalten, können diese Mechanismen allerdings nicht herangezogen werden. Dies hat einen einfachen Grund: Sowohl die Identität von lexikalischem Frame und Konstruktions-Frame (Unterabschnitt 8.2.1) als auch die Evokation des Konstruktions-Frames über Spreading-Activation-Prozesse (Unterabschnitt 8.2.2) beruhen auf der lexikalischen Evokation des Konstruktions-Frames über ein KtE des KE EREIGNIS. Wie schon in Unterabschnitt 8.1.1 erläutert, werfen Konstrukte mit unrelatierten lexikalischen Frames aus diesem Grund Probleme auf: Die Evokation des Konstruktions-Frames ist nicht mehr auf ein einzelnes KtE eines KE oder (abgesehen von einer Ausnahme bei der reflexiven WegKonstruktion) ein lexikalisch fixiertes Strukturelement wie ein KEE oder ein KorE zurückzuführen. War die Evokation des Konstruktions-Frames bei relatierten lexikalischen Frames noch im Wortsinne ,lexikalisch' orientiert, muss bei unrelatierten lexikalischen Frames nach alternativen Mechanismen gesucht werden (vgl. die rechte Seite in Abbildung 8.1 in Unterabschnitt 8.1.1).

An diesen ersten Beobachtungen ist zu erkennen, dass der Einflussfaktor der formalen Abstraktheit einer Konstruktion (Unterabschnitt 8.1.3) bei unrelatierten lexikalischen Frames nun eine zentrale Rolle spielt. Mit ihm entscheidet sich, ob die Evokation des Konstruktions-Frames durch ein lexikalisch fixiertes Strukturelement wie ein KEE oder KorE evoziert werden kann. Es ist offensichtlich, dass dies nur für Konstruktionen, die über solche Elemente verfügen, überhaupt in Erwägung gezogen werden kann. Für die reflexive Bewegungskonstruktion und die reflexive Partikelverbkonstruktion habe ich die entsprechende Frage, ob bei ihnen die Evokation des Konstruktions-Frames in dieser Form möglich ist, allerdings in Unterabschnitt 8.1.1 bereits verneint. Eine ausführlichere Begründung dafür steht jedoch noch aus. Diese möchte ich in diesem Abschnitt nachholen, wobei ein weiterer, bisher noch nicht diskutierter Mechanismus hinzukommen muss, um jene Konstrukte der reflexiven Bewegungskonstruktion und der reflexiven Partikelverbkonstruktion zu erfassen, in denen die Evokation nicht über ein lexikalisch fixiertes Strukturelement erfolgen kann.

In diesem Abschnitt soll es deshalb zuerst um die Evokation des Konstruktions-Frames durch lexikalisch fixierte Strukturelemente gehen. In Unterabschnitt 8.3.1 möchte ich diskutieren, ob für die drei untersuchten Konstruktionen eine 
Evokation des Konstruktions-Frames durch deren KEE infrage kommt. Da das Ergebnis, wie bereits erwähnt, negativ ausfällt, sollen dabei vor allem die Gründe im Vordergrund stehen, warum dies für den Fall der reflexiven Bewegungskonstruktion, der reflexiven Partikelverbkonstruktion und der reflexiven Weg-Konstruktion nicht möglich ist. In Unterabschnitt 8.3.2 soll die Frage dann auf das KorE der reflexiven Weg-Konstruktion übertragen werden, die unter den drei untersuchten Konstruktionen die einzige darstellt, bei der eine Evokation des Konstruktions-Frames über ein lexikalisch fixiertes Strukturelement plausibel erscheint. Unterabschnitt 8.3.3 schließlich widmet sich derjenigen Menge an Konstrukten, die übrig bleiben und deren Evokation des Konstruktions-Frames nicht über ein einzelnes Strukturelement, weder ein KtE eines KE noch ein KEE oder KorE erklärt werden kann. Für sie muss stattdessen angenommen werden, dass die Evokation über die syntagmatische Kombination mehrerer Strukturelemente, insbesondere von KtE und KEE, verläuft.

\subsubsection{Evokation durch ein KEE}

Während die Evokation des lexikalischen Frames für die drei untersuchten Konstruktionen den KtE des KE EREIGNIS vorbehalten ist und nur für den Fall, dass der lexikalische Frame mit dem Konstruktions-Frame identisch ist oder es sich um einen relatierten lexikalischen Frame handelt, für die Evokation des Konstruktions-Frames verantwortlich gemacht werden kann (vgl. Unterabschnitte 8.1.2 sowie Abschnitt 8.2), sind lexikalisch spezifizierte Strukturelemente einer Konstruktion, wie bereits in Unterabschnitt 8.1.3 festgehalten, für die ,lexikalische“ Evokation des Konstruktions-Frames, die deutlich von derjenigen des lexikalischen Frames unterschieden ist, grundsätzlich als relevanter einzustufen. Aus diesem Grund erscheint es angebracht, die KEE und KorE der drei untersuchten Konstruktionen daraufhin zu überprüfen, ob sie für die Evokation des Konstruktions-Frames infrage kommen. In diesem Unterabschnitt soll es zunächst um die KEE aller drei Konstruktionen gehen. Ich betrachte zunächst das für alle drei Konstruktionen als KEE bezeichnete Strukturelement, das als Reflexivum oder Reziprokpronomen instanziiert wird. Im Anschluss daran soll ein gesonderter Blick auf die reflexive Partikelverbkonstruktion geworfen werden, die, wie bereits in Unterabschnitt 3.2.2 erläutert, über ein zweites KEE verfügt, das ich als RichtUNG bezeichne. ${ }^{16}$

16 Wenn also allgemein lediglich von den KEE der drei Konstruktionen die Rede ist, ist damit das durch ein Reflexivum oder Reziprokpronomen instanziierte KEE gemeint. Wenn ich mich auf 
Warum ist intuitiv gesehen gerade das KEE ein Kandidat für die Evokation des Konstruktions-Frames, abgesehen von dessen Rolle bei der Bestimmung der formalen Abstraktheit einer Konstruktion (dazu Unterabschnitt 5.1.2), die wiederum einen wesentlichen Einflussfaktor für den Mechanismus der Evokation des Konstruktions-Frames darstellt (vgl. Unterabschnitt 8.1.3)? Zur Beantwortung dieser Frage soll ein Blick auf die in Kapitel 6 eingangs diskutierte Gegenüberstellung der Strukturelemente von Konstruktionen und von Frames dienen, wie sie von Lee-Goldman \& Petruck (2018: 36) vertreten wird. KEE werden in dieser Gegenüberstellung (wie gesehen, fälschlicherweise) mit LE bzw. FEE verglichen, was vor allem aufgrund ihrer Funktion als ,evozierend' geschieht. Ohne Zweifel ist ihre Funktion des ,Evozierens“ jedoch etwas, das beide Arten von Strukturelementen vereint. Die Gegenüberstellung von KEE und LE bzw. FEE suggeriert allerdings nicht nur, dass KEE allein in der Lage wären, ein Konstrukt eindeutig als Instanz einer bestimmten Konstruktion zu identifizieren, sondern sie könnten ebenso den Schluss nahelegen, dass KEE in der Lage sein können, Frames zu evozieren. Besitzt eine Konstruktion ein KEE, entsteht bisweilen der Eindruck, dass der Fall, dass das Vorhandensein eines KEE, das für die Evokation eines (Konstruktions-) Frames verantwortlich sei, der Normalfall ist, wobei das Fehlen eines KEE dieser Annahme Probleme bereitet:

It is current practice of the FrameNet construction annotation project to define a frameevoking element, i.e. an element that unambiguously characterizes the construction as such. In certain cases this is fairly straight-forward (let alone, the Xer the Yer, cf. Fillmore[, Kay \& O'Connor 1988]), whereas in other cases no element of the construction immediately suggests itself, so that even a construction in its entirety may be a frame-evoking element. (Hilpert 2009: 38)

Abgesehen von der bereits in Kapitel 6 erläuterten Feststellung, dass die Evokation bei FEE bzw. LE und KEE terminologisch betrachtet zunächst unterschiedliche Gegenstände, nämlich Frames auf der einen Seite und Konstruktionen auf der anderen Seite, betreffen, bleibt die Frage, ob KEE über diese in erster Linie terminologische Parallele hinaus tatsächlich dasselbe wie LE bzw. FEE leisten können. Mit anderen Worten: Können konstruktionsevozierende Elemente auch frame-evozierende Elemente sein?

Hierzu soll noch einmal ein Blick auf die Form des KEE der drei Konstruktionen dienen, auf die ich bereits in den Unterabschnitten 3.1.2, 3.2.2 und 3.3.2 eingegangen bin. Der Grund, warum alle drei Konstruktionen das Attribut reflexiv im Namen tragen, liegt darin, dass als KEE ein Reflexivum oder (seltener) ein Rezi-

das KEE RICHTUNG beziehe, nutze ich entsprechend letztere Bezeichnung, wenngleich durch sie nicht unmittelbar deutlich wird, dass es sich dabei ebenso um ein KEE handelt. 
prokpronomen obligatorisch ist (vgl. Unterabschnitt 7.2.1). Das Reflexivum kann, wie in den Konstrukten in (29)-(31), als ,echtes' Reflexivum sich ohne Numerusoder Genusmarkierung auftreten oder als ,reflexiv gebrauchtes‘ Personalpronomen, das je nach Numerus und Genus unterschiedliche Formen annimmt (vgl. dazu Duden 2016: 271).

(29) \{Zehntausende von Überlebenden retteten [KEE sich] auf Hügel, Bäume und Hausdächer\}, wo sie ohne Trinkwasser und Nahrungsmittel teilweise tagelang ausharrten, bis sie von Hubschraubern gerettet und in Sammellager geflogen wurden. (Archiv der Gegenwart, 2001 [2000])

(30) Nass geschwitzt stiegen sie wieder zur Straße hinauf - nachdem Staubfinger dem starrköpfigen Wagen einen letzten Tritt versetzt hatte -, kletterten über die Mauer, die aussah, als wäre jeder einzelne Stein mehr als tausend Jahre alt, und \{kämpften [KEE sich] den Hang hinauf\}. (Funke, Cornelia: Tintenherz, Hamburg: Cecilie Dressler Verlag 2003, S. 226)

(31) Die zwei Sekunden, die ein Bahnsteigschaffner in St. Moritz zu spät anpfiff, addierten sich zu Nachlässigkeiten von Lokführern auf norditalienischen Strecken, \{bahnten [KEE Sich] ihren Weg zu Zeitschlampereien in Südfrankreich\}, trieben weiter, Ungenauigkeiten, überall, ach, was soll das schon machen ... Sekunden addierten sich, zogen ihre Bahn negativer Informationen durch die Verkehrssysteme, sammelten ihresgleichen um sich, ohne Unterlaß. (Kopetzky, Steffen: Grand Tour, Frankfurt am Main: Eichborn 2002, S. 434)

Nun stellt sich also die Frage, ob ein Reflexivum oder Reziprokpronomen ${ }^{17}$ in der Lage ist, überhaupt einen Frame zu evozieren und wenn ja, auch den Konstruktions-Frame. Diese Frage ist bereits bei der Entwicklung von Methoden zur Benennung und Definition der Strukturelemente in Abschnitt 7.3 aufgetreten und kann simpel beantwortet werden. Das KEE der drei Konstruktionen ist, ähnlich wie die KtE der KE BEWEgENDES und Weg bzw. 〈WEG〉 sowie das KEE Richtung der reflexiven Partikelverbkonstruktion, als durch FE von lexikalischem Frame und/oder Konstruktions-Frame semantisch motiviert zu betrachten. Es wird deshalb auch nicht, wie etwa das KE EREIGNIs, über mögliche Frames, die es zu evozieren in der Lage wäre, benannt und definiert. Aus diesem Grund habe ich das KEE bereits in

17 Für das Reziprokpronomen einander käme eventuell der Frame Reciprocali ty infrage, der in FrameNet 1.7 allerdings nicht-lexikalisch ist, dem also keine LE zugeordnet sind (vgl. dazu Ruppenhofer et al. 2016: 87). Ich klammere das Reziprokpronomen deshalb aus der folgenden Betrachtung aus, zumal Reciprocality ebenso wenig als Konstruktions-Frame der drei untersuchten Konstruktionen infrage kommt wie ein potenziell von einem Reflexivum evozierter Frame. 
Abschnitt 6.3 für die reflexive Bewegungskonstruktion auf seine semantische Motivierung durch FE untersucht und es in die Betrachtung der Strukturparallelen von Konstruktionen und Frames eingeschlossen. Wenngleich, wie in Abschnitt 6.3 eingangs erläutert, die Zuweisung semantischer Rollen zu Reflexiva ein in der bisherigen Forschung nicht einheitlich behandeltes Problem ist, erscheint diese Lösung sinnvoller als diejenige, dem KEE der drei untersuchten Konstruktionen einen Frame zuzuschreiben, den es evozieren könnte.

Unabhängig davon ist es natürlich möglich, die frame-evozierende Leistung von Reflexiva zu untersuchen. Als erste Annäherung bietet es sich an, nachzusehen, ob Reflexiva in FrameNet 1.7 als LE verzeichnet sind oder nicht. Die Antwort fällt negativ aus: LE mit dem Zweitglied self oder selves sind dort nicht verzeichnet. Hierbei sind allerdings drei Dinge zu beachten, die die semantischen Charakteristika von Reflexiva betreffen, aber auch durch die Natur FrameNets als Ressource für das Englische bedingt sind.

1. Reflexiva kommen nicht zwangsläufig eigenständige semantische Eigenschaften zu, wie sie für Verben, Nomen oder andere Wortarten, die bei FrameNet üblicherweise als LE angesetzt werden, postuliert werden können - am ehesten ist dies noch bei Reflexiva mit, reflexiv gebrauchten' Verben möglich, nicht aber bei ,echt reflexiven` Verben (vgl. Geniušienè 1987: 30).

2. Reflexivkonstruktionen sind im Englischen grundsätzlich deutlich seltener als im Deutschen (vgl. Wagner 1977: 68-69; Oya 2002: 968; Zifonun 2003: 67; Ágel 2017: 343-344; Welke 2019: 435-438; Mortelmans \& Smirnova 2020: 62), was die Wahrscheinlichkeit, ein Reflexivum als LE in FrameNet anzutreffen, nochmals deutlich senkt.

3. Das Englische verfügt, anders als das Deutsche, nicht über zwei getrennte Formen für ein ,einfaches' (z.B. sich) und ein ,emphatisches' Reflexivum (z.B. sich selbst), sondern dort fallen beide in einer Form zusammen (z.B. herself), die dann funktional äquivalent zur ,starken' Form ist, wie sie etwa im Deutschen zu finden ist (vgl. König \& Siemund 2000: 233; Zifonun 2003: 24-25, 29-30; Kaufmann 2004: 191-193; König \& Gast 2008: 6).

Dies führt zu der Schlussfolgerung, dass die KEE der drei untersuchten Konstruktionen nicht als frame-evozierend betrachtet werden können. ${ }^{18}$ Damit kommen sie auch nicht für die Evokation des Konstruktions-Frames infrage. Dies gilt umso mehr, da ein Reflexivum, selbst wenn es als LE in FrameNet verzeichnet wäre,

18 Es handelt sich bei ihnen also um „single-word LUs [that] cannot function as frame-evoking elements because they alone are not able to account for the meaning of the constructions they occur in“ (Czulo, Ziem \& Torrent 2020: 2). 
kaum als LE des Konstruktions-Frames Motion vorstellbar ist. ${ }^{19}$ Die Evokation des Konstruktions-Frames kann also für alle drei Konstruktionen nicht über das KEE erfolgen, sondern muss durch andere Mechanismen geschehen.

Bevor ich jedoch zu weiteren Mechanismen komme, sei ein Blick auf das zweite KEE der reflexiven Partikelverbkonstruktion geworfen: das KEE RICHTUNG. Die reflexive Partikelverbkonstruktion ist unter den drei untersuchten Konstruktionen die einzige, die über ein zweites KEE verfügt. Wenn also bereits das KEE, das durch ein Reflexivum instanziiert wird, als nicht frame-evozierend klassifiziert wurde, soll für Richtung zumindest überprüft werden, ob sich diese Beobachtung darauf überträgt oder ob zumindest dieses KEE als frame-evozierend angesehen werden kann.

Das KEE RICHTUNG der reflexiven Partikelverbkonstruktion wird durch eine Verbpartikel instanziiert, die entweder in Distanz- oder Kontaktstellung zum Basisverb steht, das als KtE des KE EREIGNIS instanziiert wird. ${ }^{20}$ Beispiele für Instanzen des KEE RICHTUnG sind durch, (hin)ein oder vor. Die Belege in (32) zeigen Konstrukte mit Distanzstellung der Verbpartikel, während die Belege in (33) Konstrukte mit Kontaktstellung illustrieren sollen. Zur Übersicht sind sowohl das Basisverb, also die KtE des KE EREIGNIS, als auch das KEE RICHTUNG annotiert.

a. Der Gestank, der trotz des Regens in der Luft liegt, ist so widerlich, daß sogar die Fliegen fortbleiben; nur die Ratten vermehren sich, $\{[$ EReignis fressen] sich [Richtung durch]\} und bauen sich Nester in den fauligen Eingeweiden. (Schrott, Raoul: Tristan da Cunha oder die Hälfte der Erde; Hanser Verlag 2003, S. 256)

b. Zwar standen pro Wohnung zwei Kinderzimmer bereit, was einer Aufforderung zur Fortpflanzung gleichkam, doch \{[EREIGNIs mieteten]

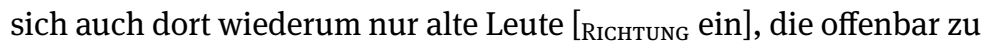
schwach und hinfällig waren, um gegen die reizlosen schuhkartonförmigen Siebziger-Jahre-Räume aufzubegehren\}. (Düffel, John von: Houwelandt, Köln: DuMont Literatur und Kunst Verlag 2004, S. 20)

19 An dieses Problem müssten sich grundsätzlichere Fragen danach anschließen, ob es Wortarten gibt, die nicht (mehr) frame-semantisch beschrieben werden können - ein Aspekt, der aufgrund des Fokus auf prädizierende Wortarten bei FrameNet tendenziell vernachlässigt wird und Konsequenzen für den Entwurf einer Frame-Semantik insgesamt hätte (vgl. dazu Busse 2012: 149153, 156-160).

$20 \mathrm{Zu}$ der konkreten Bandbreite an Verbpartikeln, die dafür infrage kommen, vgl. Tabelle 3.5 in Unterabschnitt 3.4.2 sowie Tabelle 8.5 in diesem Unterabschnitt. 
c. Obwohl nach den Untersuchungen der Uno und der OECD fast alle EU-Staaten Einwanderer brauchen werden, [[EREIGNis wagt] sich im Augenblick niemand [Richtung vor]\}. (Die Zeit, 02.03.2000, Nr. 10)

(33) a. Es sieht aus wie ein Körper, die Lunge des Magma sich gegen die Rippen pressend, ein und ausatmend, pochend wie Blut, anschwellend, sich aufstauend und das Land darüber anhebend, es auseinanderziehend, bis es dünn wird wie Haut, schließlich reißt und \{geschmolzenes Gestein sich nun [RIchtung durch][EREIGNis schmilzt]\} und sich in die Grabenbrüche ergießt, von Neufundland über Brasilien bis nach Westafrika. (Schrott, Raoul: Tristan da Cunha oder die Hälfte der Erde; Hanser Verlag 2003, S. 522)

b. \{Wer sich - zu Fuß oder mit dem Mietwagen - [Richtung hinein][EREIGNIS traut]\}, sollte freilich immer auf Überraschungen eingestellt sein. (Die Zeit, 27.01.2000, Nr. 5)

c. Aber kann man es jemandem ankreiden, wenn \{er sich in der Dunkelheit [RIchtung vor][EReIGNis tastet]\} und duckt und flüstert, statt zu rennen und zu brüllen und wild um sich zu schlagen?“ (Krausser, Helmut: Eros, Köln: DuMont 2006, S. 256)

Was motiviert die mögliche Annahme, Verbpartikeln könnten, unabhängig vom Basisverb, Frames evozieren? Wie Lüdeling (2001: 145-155) zeigt, können bestimmte Klassen von Partikelverben als komplexe Prädikate analysiert werden: Der Verbpartikel kommt hierbei der Status eines sekundären Prädikats (neben dem Basisverb als primärem Prädikat) zu. Dieser Ansatz lässt sich für die Frage nach der Möglichkeit der Evokation des Konstruktions-Frames nutzbar machen. Die Analyse des Basisverbs als (primäres) Prädikat ist konstruktionssemantisch bereits dadurch sichergestellt, dass die KtE des KE EREIGNIS als LE lexikalischer Frames gelten, also für die Evokation des lexikalischen Frames verantwortlich gemacht werden können (vgl. dazu bereits Unterabschnitt 7.3.1 sowie Abschnitt 8.2). Sie sind, das zeigen Stiebels (1996: 38) und Müller (2002: 253), als Köpfe der Partikelverben zu verstehen.

Die Frage, ob Partikeln überhaupt als sekundäre Prädikate infrage kommen, präsupponiert eine Entscheidung darüber, ob es sich dabei um Instanzen von FE handelt oder sie im weitesten Sinne als frame-evozierende LE verstanden werden können. In der generativen und lexikalistisch orientierten Partikelverbforschung dreht sich diese Entscheidung um die Frage, ob, wie Lüdeling (2001: 135) resümiert, Partikeln als prädizierende Argumente oder theta-markierte Komplemente 
zu verstehen sind..$^{21}$ Lüdeling (2001: 6, 135) argumentiert sodann dafür, Partikeln nie als theta-markierte Komplemente, sondern als Argumente zu verstehen und damit ihre prädikative Leistung ernstzunehmen. Frame-semantisch reformuliert betrifft das die Frage danach, ob Partikeln als LE und damit eigenständige (wenngleich sekundäre) Prädikate angesehen werden können oder ob sie schlichte Instanzen von FE sind und damit nicht zuvorderst frame-evozierenden Status besitzen.

Dass dieses Problem weniger gewichtig ist als es die (formal orientierte) Partikelverbforschung erscheinen lassen mag, hat im Grundsatz bereits aus satzsemantischer Perspektive von Polenz (2008: 125-126) gezeigt, indem er strikt davon ausgeht, dass Komplemente (in der Terminologie von Lüdeling 2001: 135, in seiner Terminologie: Referenzstellen) nicht nur referieren, sondern (zugleich) prädizieren können. Seine Analyse ist grundsätzlich mit derjenigen von Partikelverben als komplexen Prädikaten und damit Partikeln als sekundären Prädikaten kompatibel: „Solche Referenzprädikationen gehören nicht mit zum Hauptprädikat des betreffenden Satzinhalts “ (von Polenz 2008: 125). Hinter dem, was von Polenz hier beschreibt, verbirgt sich ein frame-semantisches Konzept, das empirisch bisher kaum durchdrungen ist: ${ }^{22}$ die Rekursivität von Frames. Es ist in Ansätzen bereits im kognitionswissenschaftlichen Frame-Modell von Minsky (1975) angelegt und wird im kognitionspsychologischen Frame-Modell von Barsalou (1992b: 21) erstmals expliziert: ${ }^{23}$ „Because frames also represent the attributes, values, structural invariants, and constraints within a frame, the mechanism that constructs frames builds them recursively.“ Busse (2012) definiert Rekursivität Barsalou folgend wie folgt:

Als Strukturen aus Konzepten sind Frames, da Konzepte selbst wieder Frames sind, immer schon Strukturen aus Frames. Jedes Attribut, [= FE, A.W.] jeder Wert, jede Relation und jedes angeschlossene Element [...] ist selbst wieder ein Frame. Auf der Ebene einer prinzipiellen Betrachtung sind Frames daher aufgrund ihrer grundsätzlichen Rekursivität prinzipiell unabschließbar. (Busse 2012: 613)

Bezogen auf die Frage nach der Evokation des Konstruktions-Frames ließe sich dies dergestalt reformulieren, dass Instanzen von FE, die zunächst dem von einem Hauptprädikat evozierten Frame angehören, wiederum Frames evozieren können,

21 Lüdeling (2001: 135) kritisiert damit Stiebels (1996), die ihrer Ansicht nach den Begriff des Arguments in irreführender Weise (nämlich für theta-markierte Komplemente) gebraucht. Wie ich gleich argumentieren werde, ist dieses Problem weniger drastisch als es den Anschein haben mag.

22 Vielleicht weil es „möglicherweise bei Linguisten eher unbeliebt ist“ (Busse 2012: 611).

23 Vgl. dazu auch die Darstellungen von Busse (2012: 255, 276, 371, 379). 
wodurch sie als sekundäre Prädikate verstanden werden können. Auf Partikelverben angewendet heißt dass, dass für die Partikel nicht die binäre Entscheidung getroffen werden muss, ob es sich dabei - in der Terminologie von Lüdeling (2001: 6, 135) - um ein (theta-markiertes) Komplement, also die Instanz eines FE, oder ein (prädizierendes) Argument, also ein sekundäres Prädikat, handelt. Nimmt man Rekursivität von Frames an, kann eine Partikel beides sein - lediglich die Analyseperspektive entscheidet darüber, ob das eine oder das andere im Vordergrund steht.

Um nun herauszufinden, ob die Verbpartikeln, die das KEE RICHTUNG der reflexiven Partikelverbkonstruktion instanziieren, Frames evozieren können, ist ganz analog zur Vorgehensweise bei den KEE aller drei Konstruktionen zu prüfen, ob in FrameNet 1.7 entsprechende LE verzeichnet sind. In Tabelle 8.5 sind daher für alle 23 Partikeln, die zur Datenauswahl für die reflexive Partikelverbkonstruktion gehören (dazu Unterabschnitt 3.4.2), mögliche äquivalente in FrameNet verzeichnete LE sowie die Frames, die sie evozieren, zusammengestellt.. ${ }^{24}$

Wie in Tabelle $8.5 \mathrm{zu}$ sehen ist, sind zunächst nicht für alle Partikeln äquivalente LE vorhanden, der Großteil jedoch findet sich in FrameNet wieder. Sieht man sich nun die Frames an, zu denen diese LE gehören, so fällt auf, dass sich unter ihnen nicht Motion, also der angenommene Konstruktions-Frame für die reflexive Partikelverbkonstruktion, findet. Hinzu kommt, dass unter diesen Frames ebenso keine zu finden sind, die zu Motion in Frame-Nähe stehen, also als relatierte Frames einzustufen wären. Dies ist deshalb wichtig, weil dadurch ausgeschlossen werden kann, dass eine Evokation des Konstruktions-Frames ausgehend von einem relatierten Frame über den Mechanismus der Spreading Activation stattfinden kann (dazu Unterabschnitt 8.2.2). Dies führt gleichzeitig dazu, dass die Instanzen des KEE RichtUng nicht als Grundlage für eine Methode zur Ermittlung des Konstruktions-Frames taugen, da die Frames, die sie evozieren, nicht über Framezu-Frame-Relationen mit dem Konstruktions-Frame verbunden sind (dazu weiterhin Unterabschnitt 8.5.2). Es lässt sich also festhalten, dass die Instanzen des KEE RICHTUNG der reflexiven Partikelverbkonstruktion zwar, anders als diejenigen der als Reflexivum instanziierten KEE aller drei Konstruktionen, in der Lage sind, Frames zu evozieren, unter diesen Frames aber weder der Konstruktions-Frame selbst noch ein zu ihm relatierter Frame ist. Deshalb kann auch für dieses KEE RICHTUNG das Fazit gezogen werden, dass es ebenso wie die KEE aller drei Konstruktionen nicht in der Lage ist, den Konstruktions-Frame zu evozieren.

24 Wie für alle äquivalenten LE in FrameNet gilt auch hier, dass die englischen Entsprechungen keine kontextadäquaten Übersetzungsäquivalente darstellen müssen, sondern lediglich eine Annäherung an die lexikalische Bedeutung der Partikel darstellen. 
Tab. 8.5: Verbpartikeln als Instanzen des KEE RICHTUNG der reflexiven Partikelverbkonstruktion und die von ihnen evozierten Frames in FrameNet 1.7

\begin{tabular}{lll}
\hline Deutsche Partikel & LE in FrameNet 1.7 & Frame \\
\hline ab & - & - \\
an & up.prep & Locative_relation \\
auf & on.prep & Spatial_contact \\
aus & off.prep & Spatial_contact \\
bei & past.prep & Locative_relation \\
durch & - & - \\
ein & in.prep & Interior_profile_relation \\
fort & - & - \\
frei & - & - \\
heim & home.adv & Spatial_co-location \\
her & in.prep & Interior_profile_relation \\
hin & on.prep & Spatial_contact \\
hinter & behind.prep & Non-gradable_proximity \\
hoch & up.prep & Locative_relation \\
mit & with.prep & Accompaniment \\
nach & to.prep & Goal \\
über & over.prep & Non-gradable_proximity \\
um & - & - \\
unter & down.adv & Direction \\
vor & forward.adv & Direction \\
weg & - & - \\
zu & to.prep & Goal \\
zurück & - & - \\
\hline & &
\end{tabular}

Vor dem Hintergrund dieser Befunde muss allerdings festgehalten werden, dass sie nur für die drei untersuchten Konstruktionen Geltung beanspruchen können. Es soll damit nicht gesagt werden, dass KEE grundsätzlich nicht für die Evokation des Konstruktions-Frames verantwortlich gemacht werden können. So können bestimmte KEE anderer Konstruktionen sehr wohl einen entsprechenden Konstruktions-Frame evozieren. Ein einfacher Fall sind etwa Konditionalkonstruktionen: ${ }^{25}$

Give us an if and a was, and we have more than enough to construct a whole conditional semantics and pragmatics, without any further formal structure. (Dancygier \& Sweetser 2005: 24)

25 Für ein Beispiel aus dem Tschechischen, in dem ein Konstruktions-Frame ohne ein lexikalisch instanziiertes Element evoziert wird, vgl. Fried (2010: 98). 
Ein kurzer Blick in FrameNet 1.7 genügt, um festzustellen, dass in der Tat die Subjunktion wenn (if.scon) als LE des Frames Conditional_occurrence verzeichnet ist, der als Konstruktions-Frame einer Konditionalkonstruktion infrage kommt. Die eingangs aufgeworfene Frage, ob konstruktionsevozierende Elemente auch frame-evozierende Elemente sein können, kann also grundsätzlich bejaht werden, wenngleich sie für die drei untersuchten Konstruktionen verneint werden muss. ${ }^{26}$ In diesem Sinne betont auch Schmid (2020: 251), dass ,there is a huge variability in the ways in which syntagmatic arcs are signalled and can unfold. "So vielfältig wie die KEE von Konstruktionen beschaffen sein können, so vielfältig kann auch die Antwort auf die Frage ausfallen, ob sie für die Evokation des KonstruktionsFrames verantwortlich gemacht werden können.

Offen bleibt damit allerdings noch immer die Frage, was es - den Terminus $K E E$ wörtlich genommen - heißt, eine Konstruktion zu evozieren. Die bisherige konstruktikographische Forschung hat hierauf keine Antwort, eben weil die Parallele zwischen KEE und LE bzw. FEE vor allem eine terminologische ist (vgl. die Argumentation im Eingang von Kapitel 6). So sind bisher keine theoretischen Prinzipien der Evokation von Konstruktionen beschrieben worden, die sich von dieser terminologischen Parallele lösen und ergründen, welche Rolle ein KEE etwa für die Identifikation einer Konstruktion spielt, außer dass es sich dabei um ein lexikalisch spezifiziertes Strukturelement (im Sinne der Definition von Fillmore, Lee-Goldman \& Rhomieux 2012: 323) handelt. Für das Beispiel der reflexiven Bewegungskonstruktion, der reflexiven Partikelverbkonstruktion und der reflexiven Weg-Konstruktion, in denen das KEE ein Reflexivum darstellt, kann dieses kaum als distinktives Charakteristikum für diese Konstruktion gelten. Mit anderen Worten: Allein das Auftreten eines Reflexivums gibt keinen Anlass zur Annahme einer reflexiven Bewegungskonstruktion, reflexiven Partikelverbkonstruktion oder reflexiven Weg-Konstruktion, es kann sich genauso gut um eine andere Reflexivkonstruktion, der ein anderer Konstruktions-Frame zugrunde liegt, handeln. Aus konstruktionssemantischer Perspektive ist dieses Problem zunächst nicht von Relevanz, da die Frage nach der Evokation des Konstruktions-Frames - und nicht der Konstruktion selbst - im Vordergrund steht. Da das KEE dafür zumindest für den Fall der drei untersuchten Konstruktionen ausscheidet, müssen andere Mechanismen, nach denen die Evokation des Konstruktions-Frames insbesondere

26 Für die KEE der drei untersuchten Konstruktionen sowie das Beispiel der Konditionalkonstruktionen gilt also die Beobachtung von Schmid (2020: 250), dass ,grammatical constructions are not anchored in content words but associated with function words serving as syntagmatic signposts [etwa mit KEE zu vergleichen, A.W.] in processing“. Allerdings wäre der Schluss, dass Funktionswörter dann nicht als frame-evozierende LE infrage kommen, verfehlt, wie das Beispiel von Subjunktionen wie wenn (if.scon) zeigt. 
in Konstrukten mit unrelatierten lexikalischen Frames (vgl. die rechte Seite in Abbildung 8.1 in Unterabschnitt 8.1.1) abläuft, gefunden werden.

\subsubsection{Evokation durch ein KorE}

Lexikalisch spezifizierte Strukturelemente wie KEE und KorE determinieren nicht nur zu einem wesentlichen Teil die formale Abstraktheit einer Konstruktion (Unterabschnitt 5.1.2), sondern bestehen bisweilen aus eigenständigen LE, die Frames evozieren. Aus diesem Grund ist die formale Abstraktheit einer Konstruktion, wie in Unterabschnitt 8.1.3 argumentiert, ein wesentlicher Einflussfaktor für den Mechanismus zur Evokation des Konstruktions-Frames. Ob ein Konstruktions-Frame durch ein KEE oder KorE evoziert wird, hängt weniger mit der Frage zusammen, welches der beiden Strukturelemente die untersuchte Konstruktion beinhaltet, sondern wie es konkret ausgestaltet ist, also durch welche LE es instanziiert wird. Für die reflexive Bewegungskonstruktion, die reflexive Partikelverbkonstruktion und die reflexive Weg-Konstruktion kommt das KEE nicht zur Evokation des Konstruktions-Frames infrage, da ein Reflexivum wie sich kaum als LE von Motion vorstellbar ist (vgl. Unterabschnitt 8.3.1). Damit ist aber nicht gesagt, dass KEE grundsätzlich keine (Konstruktions-)Frames evozieren können.

Die einzige der drei untersuchten Konstruktionen, in denen ein lexikalisch spezifiziertes Strukturelement für die Evokation des Konstruktions-Frames infrage kommt, ist die reflexive Weg-Konstruktion. Dies liegt daran, dass sie neben dem Reflexivum als KEE, das auch die anderen beiden untersuchten Konstruktionen besitzen, noch über ein KorE verfügt. Im Falle der reflexiven Weg-Konstruktion kann dieses KorE als für die Evokation des Konstruktions-Frames zuständig erklärt werden. Das KorE der reflexiven Weg-Konstruktion besteht, wie in Unterabschnitt 3.3.2 erläutert, aus einer NP mit dem Kopf Weg oder einem semantisch verwandten Nomen sowie einem Definitartikel (34), einem Indefinitartikel (35) oder einem Possessivartikel (36) (vgl. die ähnlichen Beobachtungen von Verhagen 2003a: 345, 2003c: 232-233; Mortelmans \& Smirnova 2020: 60).

(34) Wie ein Triumphator [bahnt sich Schiller [KoRE den Weg] durch die Menschenmenge\}, eskortiert von den Würdenträgern der Universität. (Safranski, Rüdiger: Friedrich Schiller, München Wien: Carl Hanser 2004, S. 311)

(35) Ich beobachtete, wie Doreen die Hände des Pfarrers und seiner Frau abwehrte, sie ließ die beiden zurück und \{bahnte sich [KorE einen Weg] zu den Essenswagen\}. (Franck, Julia: Lagerfeuer, Köln: DuMont Literatur und Kunst Verlag 2003, S. 301) 
(36) Doch er blieb nicht stehen, sondern \{bahnte sich [KoRE seinen Weg] um den Tisch herum\}. (Düffel, John von: Houwelandt, Köln: DuMont Literatur und Kunst Verlag 2004, S. 43)

Besonders interessant ist hier der Kopf der jeweiligen NP. Das Nomen Weg ist unschwer als LE vorstellbar, die einen Frame evoziert. In der Tat verzeichnet FrameNet 1.7 dieses Nomen (way.n) als LE des Frames Self_motion und die annotierten Belege, die sich dazu finden, sind allesamt Konstrukte der way-Konstruktion. ${ }^{27}$ Obwohl, wie Bybee (2010: 3) bemerkt, das Nomen way in der way-Konstruktion zwischen einem Status als LE (mit lexikalischer Bedeutung) und grammatischem Morphem (ohne lexikalische Bedeutung) steht, ${ }^{28}$ evoziert es einen Frame. Nun entspricht Self_motion allerdings freilich nicht dem von mir für die drei untersuchten Konstruktionen angenommenen Konstruktions-Frame Motion. Bevor ich auf diesen Aspekt zurückkomme, möchte ich zunächst noch auf die Rolle der lexikalischen Frames eingehen, da ich, wie im Eingang zu diesem Abschnitt erläutert, die Evokation des Konstruktions-Frames durch ein KorE denjenigen Konstrukten zugeordnet habe, die einen unrelatierten lexikalischen Frame evozieren.

Der einzige für die reflexive Weg-Konstruktion belegte unrelatierte lexikalische Frame Cutting in dem Konstrukt in (37) lässt deutlich den Unterschied zwischen lexikalischem Frame und Konstruktions-Frame erkennen. Während der lexikalische Frame wie gewöhnlich durch eine als KtE des KE EREIGNIs instanziierte LE, in diesem Fall säbeln (etwa: cut.v) evoziert wird, wird der Konstruktions-Frame durch das KorE, genauer: das Nomen Weg, das den Kopf der NP einen Weg bildet, evoziert.

27 Vermutlich ist dies der Grund, warum Lee-Goldman \& Petruck (2018: 32-33) die wayKonstruktion unter Zuhilfenahme ebendieses Frames analysieren. Auf Self_motion als Konstruktions-Frame lässt auch die Analyse der reflexiven Bewegungskonstruktion im Schwedischen von Ehrlemark, Johansson \& Lyngfelt (2016: 818) schließen, die sie als ,a self-motion construction where an actor expressed with a reflexive traverses a path in a direction from a place or towards a goal“ beschreiben, ohne jedoch explizit auf Self_motion zu verweisen. Beide Analysen scheinen die Restriktionen, die sich gegenüber Motion dabei ergeben, nicht zu erkennen: Self_motion schränkt das FE SELF_MOVER gegenüber dem korrespondierenden FE THEME in Motion dahingehend ein, dass es auf eine belebte Entität referieren muss (vgl. dazu schon Unterabschnitt 3.3.2). Dies aber trifft für die reflexive Bewegungskonstruktion, die reflexive Partikelverbkonstruktion und die reflexive Weg-Konstruktion in einer durchaus großen Anzahl von Konstrukten nicht zu, was die Verwendung von Motion, bei dem eine ähnliche Beschränkung nicht besteht, zusätzlich rechtfertigt.

28 Vgl. dazu die Analysen von Smirnova (2018: 24) sowie Mortelmans \& Smirnova (2020: 60), dass die NP als (direktes) Objekt interpretiert werden kann. Schon bei Jackendoff (1990: 212) findet sich dieselbe Annahme für die way-NP der way-Konstruktion. 
Mein Schälmesser mit der dünnen Klinge [cutting säbelt] sich einen Weg durch die buschigen Petersilienköpfe, während ich überlege, ob es tatsächlich Köpfe oder doch Blätter oder gar Büschel heißt. (Riedel, Susanne: Eine Frau aus Amerika, Berlin: Berlin Verlag 2003, S. 106)

Durch die Tatsache, dass das KorE über alle Konstrukte der Konstruktion lexikalisch spezifiziert und (mit geringen Abweichungen) invariant bleibt, kann die Evokation des Konstruktions-Frames durch dieses KorE nicht nur für Konstrukte mit unrelatierten lexikalischen Frames wie dasjenige in (37) gelten, sondern gleichsam für alle anderen Konstrukte, also auch diejenigen mit relatierten lexikalischen Frames. Die oben zitierten Konstrukte in (34)-(36) mit der LE bahnen, die als Motion evozierend betrachtet werden kann, fallen in diese Kategorie. Ob in dem Konstrukt einer Konstruktion, deren Konstruktions-Frame durch ein KorE evoziert wird (für KEE gilt prinzipiell dasselbe) ein relatierter oder ein unrelatierter lexikalischer Frame zu finden ist, spielt für die Evokation des KonstruktionsFrames also eine untergeordnete Rolle, eben weil das KorE (und analog auch das KEE) über alle Konstrukte der Konstruktion tendenziell invariant bleibt. Für den Fall, dass der lexikalische Frame nicht nur zum Konstruktions-Frame relatiert ist, sondern, wie im Fall der LE bahnen, sogar mit ihm identisch ist, wird derselbe Frame gewissermaßen ,doppelt' evoziert, nämlich einmal über das KtE des KE EREIGNIS und einmal über das lexikalisch spezifizierte KorE. Das KorE wird hier seiner Definition gerecht in dem Sinne, dass „it enhances, or supplements, a (semantic, pragmatic, discourse-functional, or syntactic) property of the construction addressed.“ (Ziem, Flick \& Sandkühler 2019: 69). Diese ,Verstärkung“ der semantischen Eigenschaften einer Konstruktion gilt nun also nicht nur in Abgrenzung zu anderen Konstruktionen wie der reflexiven Bewegungskonstruktion (die bis auf das KorE ja strukturell mit der reflexiven Weg-Konstruktion identisch ist den Status des KE 〈WEG〉 der reflexiven Weg-Konstruktion als Nicht-Kern-KE ausgeklammert), sondern auch für die Konstruktion selbst. Sie tritt eben dann auf, wenn der lexikalische Frame nicht nur zum Konstruktions-Frame relatiert ist, sondern insbesondere dann, wenn beide identisch sind.

Die bereits in Unterabschnitt 8.2.1 festgestellte Beobachtung, dass die als KtE des KE EREIGNIS instanziierte LE bahnen für die reflexive Weg-Konstruktion typisch ist und bereits den lexikalischen Frame Motion evoziert, gibt Anlass zu der Frage, ob nun die Evokation des Konstruktions-Frames durch dieses KtE oder durch ein Bestandteil des KorE primär ist. Die reflexive Weg-Konstruktion könnte dann ähnliche Eigenschaften wie ein Funktionsverbgefüge besitzen, in dem der Großteil des semantischen Gehalts durch ein nominales Element und nicht durch 
ein Verb evoziert wird (vgl. dazu van Pottelberge 2007: 437). ${ }^{29}$ Diese Tatsache registriert auch FrameNet bei der Untersuchung von support constructions:

In some situations, however, it is a noun that provides the dominant frame; in fact, in certain styles of academic or political writing the dominant frame informing the meaning of the sentence is a noun. (Fillmore et al. 2003: 324)

Dagegen spricht allerdings die durchaus belegte Varianz in den LE, die als KtE das KE EREIGNIS instanziieren können, wenngleich neben bahnen lediglich eine weitere LE, nämlich säbeln (etwa: cut.v) für die reflexive Weg-Konstruktion belegt ist. Dass es sich bei dem lexikalischen Frame Cutting, den dieses KtE evoziert, um einen unrelatierten lexikalischen Frame handelt, ist Anlass zur Annahme, dass die reflexive Weg-Konstruktion gerade nicht einem Funktionsverbgefüge nahekommt, da in einem solchen Fall der Anteil des lexikalischen Frames an der Konstitution eines entsprechenden Konstrukt-Frames höher ist als es für ein Funktionsverbgefüge zu erwarten wäre.

Die soeben bereits angesprochene (tendenzielle) Invarianz des KorE leitet über zu einem weiteren Aspekt. Für die reflexive Weg-Konstruktion sind neben dem Nomen Weg noch zwei weitere Nomen als Kopf der NP, die als KorE dient, belegt, nämlich Pfad und Trampelpfad, wie die beiden Konstrukte in (38) und (39) zeigen (vgl. dazu bereits Unterabschnitte 3.3.2 und 5.3.2).

\{Paul mußte sich [KoRE einen Pfad] zum Holzhaus bahnen\}. (Koneffke, Jan: Paul Schatz im Uhrenkasten, Köln: DuMont Buchverlag 2000, S. 77)

$\{[$ KorE Der Trampelpfad], den Jorge sich in Jahren gebahnt hatte\}, war verschwunden, das Wasser hatte ihn genommen. (Düffel, John von: Houwelandt, Köln: DuMont Literatur und Kunst Verlag 2004, S. 293)

Anders als das Nomen Weg (way.n) evoziert das Nomen Pfad (path.n) ${ }^{30}$ nicht den Frame Self_motion, sondern den Frame Roadways. ${ }^{31}$ Diese beiden Frames haben eines gemeinsam: Sie sind relatierte Frames zu Motion. Wie schon aus Tabelle 5.12 in Unterabschnitt 5.4.3 hervorgeht, steht Self_motion in einer Frame-Nähe von +1

29 Zur konstruktionsgrammatischen Relevanz von Funktionsverbgefügen vgl. Zeschel (2008), Rostila (2012) und Heine (2020: 31-33).

30 Ich gehe davon aus, dass dies ebenso auf das Determinativkompositum Trampelpfad zutrifft, das in FrameNet 1.7 nicht als eigene LE verzeichnet ist.

31 Auch für Roadways ist in FrameNet eine LE way.n verzeichnet, allerdings wird aus dem einzigen annotierten Beleg in FrameNet 1.7 deutlich, dass es sich, anders als bei der LE way.n für Self_motion, nicht um ein Konstrukt der way-Konstruktion handelt. Deswegen bleibe ich bei der Annahme, dass das Nomen Weg in diesem Fall als LE von Self_motion zu verstehen ist. 
innerhalb der Vererbungsrelation zu Motion. Der Frame Roadways steht ebenfalls in einer Frame-Nähe von +1 zu Motion, allerdings, wie aus Tabelle 5.13 hervorgeht, innerhalb der Benutzt-Relation. Die Relatiertheit dieser beiden Frames zu Motion ist letztlich der Grund dafür, sowohl Konstrukte mit dem Nomen Weg als auch solche mit dem Nomen Pfad oder Trampelpfad als Kopf der NP, die das KorE bildet, als Konstrukte derselben Konstruktion, nämlich der reflexiven Weg-Konstruktion, anzusehen.

Damit wird nun auch deutlich, warum für die reflexive Weg-Konstruktion Motion als Konstruktions-Frame angesetzt werden muss und nicht Self_motion oder Roadways. Würde man für die Konstrukte die das Nomen Weg auf der einen Seite und die Nomen Pfad und Trampelpfad auf der anderen Seite, jeweils Self_ motion bzw. Roadways als Konstruktions-Frames ansetzen, so läge konstruktionelle Polysemie vor, nämlich eine durch unterschiedliche Konstruktions-Frames bedingte Polysemie (Unterabschnitt 5.2.1). Dies müsste dann konsequenterweise zu einem Splitting der Konstruktion führen, also müssten unterschiedliche Konstruktionseinträge für eine reflexive Weg-Konstruktion mit dem Nomen Weg als KorE-Bestandteil und eine reflexive Weg-Konstruktion mit dem Nomen Pfad oder Trampelpfad als KorE-Bestandteil (gewissermaßen also eine reflexive Pfadoder Trampelpfad-Konstruktion) angenommen werden (dazu Unterabschnitte 7.2.1 und 7.2.2). Es erscheint jedoch fraglich, ob der semantische Unterschied zwischen den Konstrukten mit diesen unterschiedlichen Nomen derart groß ist, dass die Annahme unterschiedlicher Konstruktions-Frames und damit ein Splitting der Konstruktion gerechtfertigt wäre. Hinzu kommt, dass für den Fall, dass noch andere Nomen belegbar sein sollen, möglicherweise weitere KonstruktionsFrames angenommen werden müssten, was zu einem inflationären Splitting und damit $\mathrm{zu}$ vielen unterschiedlichen Konstruktions-Frames führen würde, wovor auch Perek \& Patten (2019: 376) warnen (vgl. weiterhin Unterabschnitt 8.5.1).

Aus genau diesem Grund gehe ich weiterhin davon aus, dass der Konstruktions-Frame der reflexiven Weg-Konstruktion Motion ist. Wie aber kann Motion durch LE wie Weg, Pfad oder Trampelpfad evoziert werden? Es erscheint plausibel, dass dies durch den Mechanismus der Spreading Activation (Unterabschnitt 8.2.2) geschieht. Werden die LE, die eigentlich als die Frames Self_motion und Roadways evozierend gelten, als KorE ausgedrückt, wird, da es sich um zu Motion relatierte Frames handelt, gleichzeitig auch Motion evoziert. Dabei kann davon ausgegangen werden, dass die Stärke der Evokation von Motion durchaus hoch ist, denn wie bereits erwähnt stehen beide Frames, Self_motion und Roadways, in einer Frame-Nähe von +1 zu Motion, also einer relativ großen Frame-Nähe. Diese große Frame-Nähe spricht dafür, dass Motion als Konstruktions-Frame durch das KorE der reflexiven Weg-Konstruktion evoziert wird, auch wenn die darin instanziierten LE strenggenommen zu anderen, relatierten Frames gehören. 


\subsubsection{Evokation durch syntagmatische Kombination von KtE und KEE}

Mit den in diesem und in Abschnitt 8.2 vorgestellten Mechanismen sind bereits einige Varianten der Evokation des Konstruktions-Frames diskutiert worden, die einen beträchtlichen Teil der Konstrukte der drei untersuchten Konstruktionen abdecken. Bei der Frage danach, wie die Evokation des Konstruktions-Frames vonstatten geht, haben diese Mechanismen eines gemeinsam: Sie führen die Evokation jedes Mal auf ein einzelnes lexikalisches Element zurück, das entweder eine LE des Konstruktions-Frames selbst oder eines mit zu ihm in Frame-Nähe stehenden (lexikalischen) Frames darstellt. Nimmt man die Mechanismen für alle drei untersuchten Konstruktionen zusammen, so ergeben sich die folgenden bisher diskutierten drei Varianten.

a) Der Konstruktions-Frame wird durch ein KtE des KE EREIGNIS evoziert, das direkt eine LE von Motion darstellt. Lexikalischer Frame und KonstruktionsFrame sind also identisch (Unterabschnitt 8.2.1).

b) Der Konstruktions-Frame wird durch ein KtE des KE EREIGNIS evoziert, das eine LE eines lexikalischen Frames, der zu Motion in Frame-Nähe steht, darstellt. Die Evokation erfolgt hier über den Mechanismus der Spreading Activation (Unterabschnitt 8.2.2).

c) Der Konstruktions-Frame wird durch einen Teil des KorE der reflexiven WegKonstruktion evoziert, das eine LE eines Frames, der zu Mot i on in Frame-Nähe steht, darstellt (Unterabschnitt 8.3.2).

Während die Punkte a und b insbesondere für die reflexive Bewegungskonstruktion und die reflexive Partikelverbkonstruktion gelten, ist Punkt c der reflexiven Weg-Konstruktion vorbehalten, da diese als einzige unter den drei Konstruktionen über ein entsprechend beschaffenes KorE verfügt. Was auffällt, ist, dass allein die reflexive Weg-Konstruktion mit allen ihren Konstrukten - also jenen mit relatierten lexikalischen Frames wie jenen mit unrelatierten lexikalischen Frames durch die bisher diskutierten Mechanismen der Evokation abgedeckt ist. Für die anderen beiden Konstruktionen habe ich bisher lediglich Mechanismen zur Evokation des Konstruktions-Frames bei Konstrukten mit relatierten lexikalischen Frames diskutiert (Abschnitt 8.2). Mit anderen Worten: Die oben genannten Punkte a und b gelten allein für relatierte lexikalische Frames, während Punkt c sowohl für relatierte als auch unrelatierte lexikalische Frames gilt, allerdings eben einzig für die reflexive Weg-Konstruktion. Während die Evokation des KonstruktionsFrames bei der Menge von Konstrukten der reflexiven Bewegungskonstruktion und der reflexiven Partikelverbkonstruktion mit relatierten lexikalischen Frames also abgedeckt ist, bleiben für die beiden Konstruktionen noch insgesamt zwei Teilmengen an Konstrukten übrig: 
- Konstrukte der reflexiven Bewegungskonstruktion mit unrelatierten lexikalischen Frames;

- Konstrukte der reflexiven Partikelverbkonstruktion mit unrelatierten lexikalischen Frames.

Diese beiden Teilmengen von Konstrukten sind also, anders als die bisher diskutierten, nicht auf eine ,lexikalische“ Evokation des Konstruktions-Frames durch ein KtE des KE EREIGNIS, das KEE oder einen Teil eines (in diesen beiden Konstruktionen nicht vorhandenen) KorE zurückzuführen. In Abbildung 8.5 sind diese Verhältnisse dargestellt, wobei die bereits über für eine ,lexikalische‘ Evokation des Konstruktions-Frames erfassten Konstrukte auf der linken Seite zu finden sind, während die übrig bleibenden zwei Teilmengen der Konstrukte der reflexiven Bewegungskonstruktion und der reflexiven Partikelverbkonstruktion mit unrelatierten lexikalischen Frames auf der rechten Seite dargestellt sind. Für diese Konstrukte muss es also einen anderen Mechanismus der Evokation des KonstruktionsFrames geben.

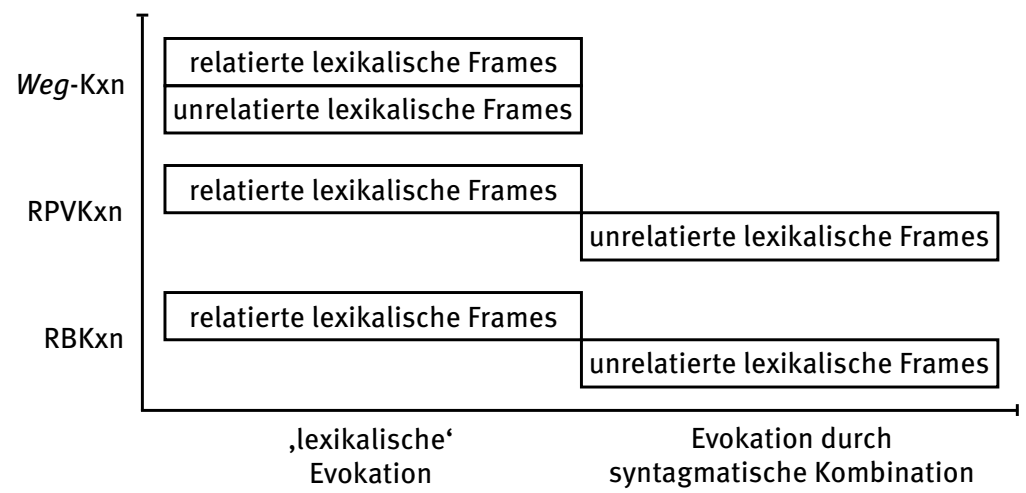

Abb. 8.5: Evokation des Konstruktions-Frames der drei untersuchten Konstruktionen nach Konstrukten mit relatierten und unrelatierten lexikalischen Frames

Anders als bei allen bisherigen Mechanismen der Evokation des KonstruktionsFrames können die beiden Teilmengen von Konstrukten auf der rechten Seite in Abbildung 8.5 nicht über eine ,lexikalische‘ Evokation des Konstruktions-Frames erklärt werden. Die entsprechenden Konstrukte verfügen über keine in einem einzigen Strukturelement instanziierte LE, die dem Konstruktions-Frame oder einem 
$\mathrm{zu}$ ihm relatierten lexikalischen Frame zuzuordnen ist. ${ }^{32}$ Für diese Konstrukte muss also ein Mechanismus angesetzt werden, der nicht auf der Evokation des Konstruktions-Frames durch ein einzelnes Strukturelement beruht.

Worin dieser Mechanismus bestehen muss, lässt sich vor dem Hintergrund der bisher diskutierten Mechanismen der Evokation des Konstruktions-Frames erahnen: Anders als die ,lexikalische“ Evokation des Konstruktions-Frames, die über lediglich ein einzelnes Strukturelement erfolgt, muss die Evokation des Konstruktions-Frames in den verbliebenen Fällen durch die Kombination mehrerer Strukturelemente erfolgen. Ganz in diesem Sinne stellen Czulo, Ziem \& Torrent (2020) bei der Analyse bestimmter Mehrworteinheiten wie Refrainfragen (tag questions) fest:

The frame-evoking power of the constructions cannot be traced back to single lexical elements but must rather be assigned to the phrase as a whole. In other words, tag questions are multi-word expressions that evoke frames in a holistic rather than a compositional fashion, in that the building blocks of the expressions cannot be considered units carrying frame-semantic information on their own. (Czulo, Ziem \& Torrent 2020: 2)

Ich möchte im Folgenden zeigen, dass nicht nur solche Mehrworteinheiten, sondern auch formal abstraktere Argumentstruktur-Konstruktionen wie die reflexive Bewegungskonstruktion und die reflexive Partikelverbkonstruktion eine vergleichbare Evokation des Konstruktions-Frames aufweisen können. Besonders relevant hierfür sind ihre KtE und KEE, weshalb ich diesen Mechanismus der Evokation des Konstruktions-Frames in der syntagmatischen Kombination dieser Strukturelemente begründet sehe.

Dass Frames nicht nur von einzelnen LE, sondern zugleich von komplexeren Einheiten wie syntaktischen Konstruktionen evoziert werden können, ist bereits in Fillmores Verstehenssemantik (Unterabschnitt 2.1.1) angelegt, wie Ziem (2008) resümiert:

Nicht nur Wörter wie kaufen, schenken, verdienen usw. implizieren schematisierte Erfahrungsund Handlungszusammenhänge. Auch das Verstehen komplexerer Ausdrücke wie Idiome und usuelle Wortverbindungen erfordert die Kenntnis eines angemessenen Kontextes, in dem diese auftreten können. [...] Ohne dass die jeweiligen Satzbedeutungen kompositionell erschlossen werden müssten, verweisen die [...] usuellen Wortverbindungen als Ganze auf typische soziale Kontexte, in denen sie geäußert werden könnten. Dieser Umstand verleiht ihnen den Status von „Konstruktionen“ (im oben erläuterten technischen Sinn). (Ziem 2008: 231-232)

32 Boas (2016: 86) weist darauf hin, dass die Untersuchung von Fällen, in denen ein Frame ohne lexikalisch zu identifizierende LE evoziert wird, ein Forschungsdesiderat ist, ignoriert dabei jedoch, dass bereits Minsky (1975: 241) ein entsprechendes Beispiel diskutiert. 
In Anlehnung der Konzeption einer symbolischen Einheit aus phonologischer und semantischer Struktur bei Langacker (z.B. 1987: 57-58) argumentiert Ziem nun, dass nicht nur einfache phonologische Strukturen in der Lage sind, Frames zu evozieren, sondern dass dies auch auf komplexere Strukturen zutrifft:

Es sind phonologische Einheiten, die derartige Aktivierungen schematischen Wissens motivieren. Solange der Verbund von phonologischer Einheit und assoziierter Bedeutung den Status einer Konstruktion hat, können phonologische Einheiten durchaus komplexer Natur sein. So ist beispielsweise mit der einfachen phonologischen Einheit [teufel] genauso eine semantische Einheit assoziiert wie mit der sehr komplexen Einheit [wenn man vom Teufel spricht]. (Ziem 2008: 232)

In Termini der Konstruktikographie ist unter einer komplexen symbolischen Struktur also eine syntaktische Konstruktion im Sinne von Langacker (1987: 82) und Diessel (2019: 11) zu verstehen, die aus mehreren Strukturelementen, also KE, KEE und/oder KorE besteht. Genauso wie etwa einzelne KtE eines KE (lexikalische) Frames evozieren können, kann dies nun auch für die Kombination solcher Strukturelemente geltend gemacht werden. Der Konstruktionsstatus dieser syntagmatischen Kombination - also die Tatsache, dass es sich dabei um ein ,FormBedeutungs-Paar' handelt - schafft die Voraussetzung, damit sie überhaupt mit einem Konstruktions-Frame assoziiert werden kann und damit letztendlich davon gesprochen werden kann, die Konstruktion ,evoziere“ diesen Frame. Schmid (2020) bezeichnet dies als syntagmatic-strengthening principle:

As the syntagmatic links within a sequence are strengthened by repetition, symbolic, paradigmatic, and pragmatic connections associated with the component parts are weakened, while symbolic, paradigmatic, and pragmatic associations of the sequence become stronger. (Schmid 2020: 236)

Wie kann man sich die Evokation eines Konstruktions-Frames über die syntagmatische Kombination mehrerer Strukturelemente vorstellen? Zunächst sei dafür an die Thesen der Diagrammatizität von Sprache bei Haiman $(1980,1983,1985)$ erinnert, auf die ich in Unterabschnitt 6.1.2 hingewiesen habe. Für die beiden Mengen an verbliebenen Konstrukten der reflexiven Bewegungskonstruktion und der reflexiven Partikelverbkonstruktion lässt sie sich an dieser Stelle noch einmal nutzbar machen. Tritt in einem Konstrukt-Frame ein unrelatierter lexikalischer Frame auf, so wird dieser in vielen Fällen durch eine LE evoziert, die distributionell kaum als ,reflexiv' einzustufen ist. So sind LE wie arbeiten (40) oder einkaufen (41), die die unrelatierten lexikalischen Frames Work bzw. Shopping evozieren, traditionell kaum als ,reflexive‘ oder auch nur ,reflexiv gebrauchte“ Verben einzustufen (vgl. dazu auch Unterabschnitt 6.1.2). 
(40) [work Arbeitete] sich durch ein 14 Pfund schweres Handbuch, das er vom FBI aus Washington erhalten hatte (für die 70 Mark Luftfracht musste er seine Wirtin anpumpen), und schrieb ganz wie sein Vorbild Karl May über ein Land, das er nie mit eigenen Augen gesehen hatte. (Die Zeit, 06.04.2000, Nr. 15)

(41) Der reine Mobilfunker startete zunächst in Großbritannien durch, wurde dort schnell zum Marktführer und [shopping kaufte] sich in aller Welt ein; meist allerdings mit Minderheitsbeteiligungen. (Die Zeit, 10.02.2000, Nr. 7)

Diagrammatisch interessant daran ist also die distributionell unübliche Kombination aus einer solchen LE und einem Reflexivum. Haiman (1983: 795-799, 1985: 142-143) und Barlow (2000: 326) zufolge ist eine solche Kombination als Zeichen für ein Vorhandensein und die Verbindung zweier Konzepte, im konstruktionssemantischen Sinne also Frames, die aufgrund eines Blending-Prozesses einen ,neuen' Frame, eben den Konstrukt-Frame, bilden (vgl. auch Hampe \& Schönefeld 2003: 246-247, 2006: 128). Das dadurch entstehende komplexe Zeichen kann durch seine Fähigkeit, einen Frame zu evozieren, als indexikalisch betrachtet werden (vgl. Ziem 2008: 233, 2014b: 199). Wichtig ist nun, dass nicht ein zusätzliches Element allein verantwortlich dafür ist, dass der Konstruktions-Frame evoziert wird - dies habe ich in Unterabschnitt 8.3.1 für das KEE der drei untersuchten Konstruktionen ja bereits gezeigt -, sondern dass die Kombination mehrerer Elemente, also etwa der LE, die den unrelatierten lexikalischen Frame evoziert, mit einem Reflexivum als KEE, die Evokation des Konstruktions-Frames leisten kann. Erst die Kombination mehrerer Strukturelemente in einem Konstrukt spannt, um eine Metapher von Schmid (2020: 65-66) zu verwenden, einen ,syntagmatischen Bogen' (syntagmatic arc) über mehrere Strukturelemente und letztlich das gesamte Konstrukt auf. Im Falle von Konstrukten der reflexiven Bewegungskonstruktion und der reflexiven Partikelverbkonstruktion sind es, wenn an ihren KonstruktFrames unrelatierte lexikalische Frames beteiligt sind, diese, syntagmatischen Bögen', die die Evokation des Konstruktions-Frames leisten. Jene entsteht also durch die syntagmatische Kombination von Strukturelementen.

Über die Fähigkeit einer syntagmatischen Kombination, einen KonstruktionsFrame zu evozieren, entscheidet ganz wesentlich die Art der Strukturelemente, die miteinander kombiniert werden. Ich möchte im Folgenden dafür argumentieren, dass manche Strukturelemente eher als andere dafür kriterial sind, was zugleich bedeutet, dass die Kombination, die zur Evokation des KonstruktionsFrames führt, nicht zwangsläufig alle Strukturelemente eines Konstrukts einschließen muss, sondern dass - wie oben am Beispiel der Kombination aus der LE, die den lexikalischen Frame evoziert und dem Reflexivum gezeigt - bereits die 
Kombination zweier Strukturelemente ausreichen kann, um den KonstruktionsFrame zu evozieren.

Die Strukturelemente der drei untersuchten Konstruktionen (mit Ausnahme des KE BewEGENDEs), insbesondere aber diejenigen der reflexiven Bewegungskonstruktion und der reflexiven Partikelverbkonstruktion, lassen sich nach ihrer Kombinationsrelevanz unterscheiden, nach der Relevanz also, die ihrer Kombination mit anderen Strukturelementen für die Evokation des Konstruktions-Frames zukommt. Abbildung 8.6 zeigt eine entsprechende Hierarchie der Strukturelemente nach ihrer Kombinationsrelevanz. Interessant sind hier vor allem die mittleren drei Strukturelemente, also KEE, RICHTUNG sowie das KE WEG bzw. 〈WEG〉, weshalb ich zunächst auf die beiden äußeren Strukturelemente eingehen möchte, bevor ich mich den mittleren dreien zuwende.

\section{hohe Kombinationsrelevanz}

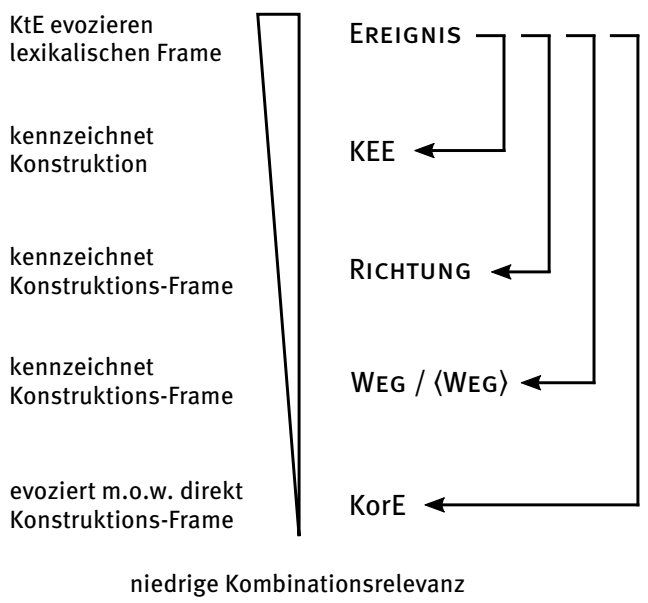

Abb. 8.6: Hierarchie der Kombinationsrelevanz von Strukturelementen in syntagmatischer Kombination zur Evokation des Konstruktions-Frames

Das KE EREIGNIS, das alle drei Konstruktionen besitzen, besitzt die höchste Kombinationsrelevanz. Dies ist insofern trivial, als dass die KtE dieses KE die lexikalischen Frames evozieren (vgl. Abschnitt 4.2 sowie Unterabschnitt 7.3.1). Ohne dieses Strukturelement wäre ein Blending aus (unrelatiertem) lexikalischen Frame und Konstruktions-Frame also gar nicht möglich. Dass es dennoch in der Hierarchie in Abbildung 8.6 erscheint, liegt darin begründet, dass es die Basis für die Kombination mit allen anderen Strukturelementen darstellt. Mit anderen Worten: Da ein KtE des KE EREIGNIS in jedem Konstrukt zu finden ist, entscheidet 
dessen Kombination mit einem anderen Strukturelement über die Evokation des Konstruktions-Frames. Die Kombinationsrelevanz aller anderen Strukturelemente wird also anhand von deren Kombination mit den KtE des KE EREIGNIS gemessen. Die Kombinationen des KE EREIGNIS mit jeweils einem anderen Strukturelement sind dabei durch die Pfeile in Abbildung 8.6 markiert.

Am anderen Ende der Hierarchie nach Kombinationsrelevanz steht das KorE der reflexiven Weg-Konstruktion. Der Grund dafür ist schlicht, dass dieses Strukturelement, wie in Unterabschnitt 8.3.2 gezeigt, in der Lage ist, den KonstruktionsFrame mehr oder weniger direkt zu selbst evozieren. Obwohl es in der reflexiven Weg-Konstruktion in Kombination mit KtE des KE EREIGNIS steht, ist diese Kombination für die Evokation des Konstruktions-Frames nicht ausschlaggebend, da die Evokation bereits durch das KorE selbst geleistet wird.

Es bleiben also die drei mittleren Strukturelemente: KEE, RICHTUNG und WEG bzw. 〈WEG $\rangle$. Aus diesem Grund spielen die KtE dieser KE sowie die KEE von reflexiver Bewegungskonstruktion und reflexiver Partikelverbkonstruktion für die Evokation des Konstruktions-Frames durch eine syntagmatische Kombination eine besondere Rolle. Eine simple Methode, die Kombinationsrelevanz dieser Strukturelemente einzuschätzen, ist, sie auf ihre Weglassbarkeit hin zu überprüfen. ${ }^{33}$ Dies lässt sich durch einen direkten Vergleich der Strukturelemente der reflexiven Bewegungskonstruktion und der reflexiven Partikelverbkonstruktion realisieren. Die prinzipielle Weglassbarkeit eines Strukturelements ist somit Anzeichen für eine geringere Kombinationsrelevanz gegenüber einem Strukturelement, das prinzipiell nicht weggelassen werden kann. Als Vergleichsgrundlage soll erneut die LE arbeiten (work.v) dienen, die den unrelatierten lexikalischen Frame Work evoziert.

Beleg (42) zeigt ein Konstrukt der reflexiven Bewegungskonstruktion. Das KEE sowie das KE WEG sind für sie obligatorisch und können nicht weggelassen werden. Die Kombination dieser beiden Strukturelemente mit dem KE EREIGNIS ist also in jedem Fall in der Lage, den Konstruktions-Frame zu evozieren.

(42) $\quad$ Sie arbeitete [KEE $\mathrm{Sich}]\left[\mathrm{WEG}_{\mathrm{E}}\right.$ durch schulterhohes Dickicht]\}; die Männer waren angeseilt, da sich unter dem Moosbewuchs tiefe Felsspalten verbargen; im stellenweise dichten Nebel hätte man sich ohne Kompaß leicht

33 Hier soll bewusst die Rede von Weglassbarkeit und nicht etwa von Null-Instanziierbarkeit sein, da Letztere präsupponiert, dass es sich bei den diskutierten Elementen um Nicht-Kern-KE handelt, die ohnehin fakultativ sind (vgl. dazu Unterabschnitt 6.4.2). Es handelt sich dabei also nicht um den Test, ob ein Strukturelement innerhalb einer Konstruktion fakultativ ist, sondern ob z.B. andere Konstruktionen existieren, die ohne dieses Strukturelement auskommen, aber denselben Konstruktions-Frame evozieren. 
verirrt. (Schrott, Raoul: Tristan da Cunha oder die Hälfte der Erde; Hanser Verlag 2003, S. 31)

Über die Kombinationsrelevanz des KEE und des KE WEG selbst sagt dies aber noch nichts aus. Erst ein Vergleich mit der reflexiven Partikelverbkonstruktion kann eine Entscheidung herbeiführen. Beleg (43) zeigt ein Konstrukt dieser Konstruktion, wobei das Nicht-Kern-KE 〈WEG〉 hier instanziiert ist. Dieses Konstrukt entspricht also strukturell nahezu demjenigen der reflexiven Bewegungskonstruktion in (42), mit dem Unterschied, dass das für die reflexive Partikelverbkonstruktion charakteristische zweite KEE RICHTUNG hinzukommt.

(43) \{Die Ermittler arbeiteten [KEE $\mathrm{sich}]\left[{ }_{\langle\mathrm{WEG}\rangle}\right.$ an den großen Zampano] [RICHTung heran]\}. (Die Zeit, 27.04.2000, Nr. 18)

Die Kombinationsrelevanz des KEE beider Konstruktionen, des KEE RICHTUNG sowie des KE WEG bzW. 〈WEG〉 lässt sich nun bestimmen, indem man sie auf ihre Weglassbarkeit hin überprüft. Die Weglassbarkeit von RICHTUNG drückt sich in dem Vergleich der Konstrukte in (42) und (43) bereits aus: Für die Evokation des Konstruktions-Frames scheint es insofern eine untergeordnete Rolle zu spielen, als dass es in Konstrukten der reflexiven Bewegungskonstruktion nicht erforderlich ist, wobei die Evokation von Motion als Konstruktions-Frame weiterhin für beide Konstruktionen plausibel erscheint.

Ebenso wie für die reflexive Bewegungskonstruktion das KEE RICHTUNG weglassbar ist, ist für die reflexive Partikelverbkonstruktion das KE 〈WEG $\rangle$ weglassbar, weshalb es als Nicht-Kern-KE einzustufen ist. Beleg (44) zeigt ein Konstrukt der reflexiven Partikelverbkonstruktion ohne Instanziierung des KE $\langle$ WEG $\rangle$.

(44) Breiring kam ins Krankenhaus, Eichhorn hatte Semesterferien, er übernahm den Laden, \{arbeitete [KEE sich] in ein paar Tagen [RIchtung ein]\}, benutzte die Karte und die Fähnchen bald so geläufig wie jedes andere einleuchtend konstruierte Werkzeug. (Kopetzky, Steffen: Grand Tour, Frankfurt am Main: Eichborn 2002, S. 154)

Festzuhalten ist nun Folgendes: Das KEE, das als Reflexivum instanziiert wird, ist in beiden Konstruktionen, sowohl der reflexiven Bewegungskonstruktion als auch der reflexiven Partikelverbkonstruktion, nicht weglassbar. Dies ist zunächst ein trivialer Fakt, der gerade zur Einstufung des Reflexivums als KEE führt. In Abbildung 8.6 ist für das KEE deshalb vermerkt, das es die jeweilige Konstruktion kennzeichnet (vgl. Unterabschnitt 8.3.1 zur Problematik der ,Evokation“ einer 
Konstruktion). ${ }^{34}$ Aufgrund seiner nicht gegebenen Weglassbarkeit muss es hinsichtlich seiner Kombinationsrelevanz unmittelbar hinter dem KE EREIGNIS stehen. Die Kombination aus einem KtE des KE EREIGNIS und dem KEE ist also kriterial, um die Evokation des Konstruktions-Frames sicherzustellen..$^{35}$ Diese hohe Relevanz des als Reflexivum instanziierten KEE trägt dem breiten Funktionsspektrum von Reflexiva Rechnung, zu dem gehört, dass das Reflexivum einem ursprünglich nicht reflexiven Verb einen zusätzlichen semantischen Aspekt hinzufügen kann (vgl. z.B. Geniušienè 1987: 28-30). Bezogen auf hohe Kombinationsrelevanz des KEE heißt dies, dass gerade die Kombination des Reflexivums mit einem KtE des KE EREIGNIS (also einem Verb) für eine wesentliche konstruktionssemantische Leistung verantwortlich gemacht werden kann. Diese Leistung besteht in der Evokation des Konstruktions-Frames.

Wie aber begründet sich die Hierarchie zwischen dem KEE RICHTUNG und dem KE Weg bzw. 〈WEG〉 hinsichtlich ihrer Kombinationsrelevanz? Sowohl die Instanzen von Richtung als auch die KtE von Weg bzw. 〈WEG〉 werden durch FE eines lexikalischen und/oder des Konstruktions-Frames motiviert (vgl. Unterabschnitte 6.2.2, 6.2.3 und 6.4.2). Sie können deshalb, wie in Abbildung 8.6 notiert, potenziell den Konstruktions-Frame kennzeichnen, aufgrund ihrer Weglassbarkeit aber nicht die Konstruktion als solche. Der Statuts von $\langle\mathrm{WEG}\rangle$ als Nicht-KernKE hängt nun wesentlich an dem Auftreten des KEE RICHTUNG. Tritt es, wie in der reflexiven Partikelverbkonstruktion, auf, muss $\langle\mathrm{WEG}\rangle$ nicht instanziiert werden, weshalb es als Nicht-Kern-KE eingestuft werden kann (vgl. dazu Unterabschnitt 6.4.2). Tritt es, wie in der reflexiven Bewegungskonstruktion, nicht auf, muss das KE WEG instanziiert werden und Kern-KE sein. Mit anderen Worten: Das KEE RICHTUNG bedingt den Status des KE $\langle\mathrm{WEG}\rangle$ als Nicht-Kern-KE. ${ }^{36}$ Da auch bei einer fehlenden Instanziierung des $\mathrm{KE}\langle\mathrm{WEG}\rangle$, wie in (44), der Konstruktions-Frame evoziert wird, sich daran bei einer Instanziierung von $\langle\mathrm{WEG}\rangle$, wie in (43), aber nichts

34 Vgl. dazu auch die Definition von KEE bei Lee-Goldman \& Petruck (2018: 26): „A Constructionevoking Element (CEE) is lexical material that is central to, or that cues the existence of, a particular construction“.

35 Vgl. auch die Analyse von Culicover \& Jackendoff (2005: 35), die für Resultativkonstruktionen „a PP that the verb would not normally license“ als kriterial zur Kennzeichnung der Konstruktion ansetzen. Interessant sind in diesem Zusammenhang auch die Ideen von Goldberg \& Herbst (2021: 301-305) zu Fragmenten von Konstruktionen, die bereits ausreichen, um eine Konstruktion als Ganzes semantisch identifizierbar zu machen und dabei selbst als konventionalisierte Konstruktionen betrachtet werden können. Inwieweit Letzteres auf die hier untersuchten syntagmatischen Kombinationen von Strukturelementen zutrifft, könnten zukünftige Forschungen eruieren.

36 Der von Olsen (1996b) eingeführte Begriff des pleonastischen Direktionals, auf den ich in Unterabschnitt 6.4.2 eingegangen bin, bringt dies anschaulich zum Ausdruck. 
ändert, stufe ich die Kombinationsrelevanz des KE WEG bzw. 〈WEG $\rangle$ niedriger als diejenige des KEE RICHTUNG ein. Damit ergibt sich die in Abbildung 8.6 dargestellte Hierarchie der Strukturelemente hinsichtlich ihrer Kombinationsrelevanz.

Diese Ergebnisse können freilich lediglich für die drei untersuchten Konstruktionen Geltung beanspruchen. Strukturelemente anderer Konstruktionen können andere Kombinationsrelevanzen aufweisen, zumal die grundsätzliche Relevanz des Mechanismus der syntagmatischen Kombination von Strukturelementen zur Evokation des Konstruktions-Frames stark davon abhängt, ob die Evokation nicht bereits durch andere Strukturelemente geleistet werden kann. Ebenso ist die Bandbreite möglicher Mechanismen der Evokation des Konstruktions-Frames damit nur für die drei untersuchten Konstruktionen abgesteckt. So vielfältig wie andere Konstruktionen beschaffen sein können, so vielfältig können auch die Mechanismen der Evokation ihrer Konstruktions-Frames beschaffen sein und es ist nicht ausgeschlossen, dass sich durch die Untersuchung anderer Konstruktionen weitere Mechanismen finden lassen, die über diejenigen in diesem und dem vorangegangenen Abschnitt 8.2 diskutierten hinausgehen.

\subsection{Methodologische Präliminarien bei der Ermittlung des Konstruktions-Frames}

Jegliche Frage nach der Evokation eines Konstruktions-Frames kann kaum beantwortet werden, wenn der Konstruktions-Frame einer Konstruktion unbekannt ist. Den unterschiedlichen Mechanismen zur Evokation des Konstruktions-Frames (Abschnitte 8.2 und 8.3) müssen also methodologische und methodische Überlegungen an die Seite gestellt werden, die der Ermittlung des Konstruktions-Frames dienen. Warum kommt gerade Motion als Konstruktions-Frame für die reflexive Bewegungskonstruktion, die reflexive Partikelverbkonstruktion und die reflexive Weg-Konstruktion infrage? Was noch fehlt, sind konkrete Methoden zur Ermittlung des Konstruktions-Frames, die, wie ich in diesem Abschnitt zeigen möchte, jedoch nicht völlig losgelöst von den in den Abschnitten 8.2 und 8.3 diskutierten Mechanismen der Evokation des Konstruktions-Frames betrachtet werden können. Vielmehr bestehen Zusammenhänge zwischen diesen Mechanismen und insbesondere deren Einflussfaktoren der Frame-Nähe (Unterabschnitt 8.1.2) und der formalen Abstraktheit einer Konstruktion (Unterabschnitt 8.1.3).

Eine Vorbemerkung, auf die ich bereits in Unterabschnitt 2.1.3 hingewiesen habe, sei an dieser Stelle wiederholt. Bei der Ermittlung eines KonstruktionsFrames kann stets nur auf diejenigen Daten zurückgegriffen werden, die in FrameNet, hier: im Daten-Release 1.7, dokumentiert sind. Da FrameNet keine lücken- 
lose Erfassung von Frames für eine Einzelsprache darstellt, kann es somit stets vorkommen, keinen passenden Frame für eine gegebene sprachliche Einheit, sei es eine LE oder eine syntaktische Konstruktion, finden zu können. Dies begründet allerdings keine grundsätzlichen Auswirkungen auf die Plausibilität der ,Bedeutunghaltigkeit‘ einer LE oder einer Konstruktion, sondern ist ein rein methodologisches Problem der Abdeckung von FrameNet. Für die drei untersuchten Konstruktionen indes ist jedoch eine Anwendung der FrameNet-Daten, insbesondere hinsichtlich der Ermittlung des Konstruktions-Frames, ohne Einschränkungen möglich.

Bevor ich die einzelnen Methoden thematisiere, möchte ich in diesem Abschnitt deshalb zunächst auf einige methodologische Präliminarien eingehen, die die Wahl der Methode zur Ermittlung des Konstruktions-Frames steuern und Voraussetzungen dafür darstellen, die Methoden überhaupt anwenden zu können. In Unterabschnitt 8.4.1 möchte ich auf den Zusammenhang zwischen den in den Abschnitten 8.2 und 8.3 diskutierten Mechanismen der Evokation des Konstruktions-Frames und der Wahl der Methode $\mathrm{zu}$ dessen Ermittlung eingehen. Wie sich zeigen wird, richtet sich die Frage nach einer geeigneten Methode zur Ermittlung des Konstruktions-Frames insofern wesentlich nach den Mechanismen von dessen Evokation, als dass etwa Konstruktionen mit einer großen Anzahl von Konstrukten auf Basis relatierter Frames andere Methoden erfordern als solche, die - wie die drei untersuchten Konstruktionen - eine größere Anzahl von Konstrukten mit unrelatierten Frames beinhalten. Nicht alle diskutierten Methoden lassen sich an den drei untersuchten Konstruktionen gleichermaßen aufzeigen und die Relevanz jeder einzelnen Methode unterscheidet sich durchaus von Konstruktion zu Konstruktion. Im Rahmen der hier thematisierten methodologischen Präliminarien zeigt sich, warum. Neben der Betonung der Rolle der Evokationsmechanismen und deren Einflussfaktoren soll darüber hinaus die Frage beantwortet werden, welche korpuslinguistischen Methoden bei der Ermittlung des Konstruktions-Frames hilfreich sein können. In Unterabschnitt 8.4.2 möchte ich deshalb dafür argumentieren, dass die Betrachtung der ambigen Formseite einer Konstruktion - für welche etwa die reflexive Bewegungskonstruktion ein anschauliches Beispiel darstellt - nicht nur bei der initialen Identifikation relevanter Konstrukte und deren Unterscheidung von Falschpositiven eine Rolle spielt (vgl. dazu Unterabschnitt 3.4.2), sondern dass dadurch eine methodologisch äußerst wichtige Voraussetzung für die Ermittlung des Konstruktions-Frames geschaffen wird: die Ermittlung potenzieller relatierter lexikalischer Frames. 


\subsubsection{Zur Rolle der Evokationsmechanismen}

Die eher theoretischen Fragen nach der Evokation des Konstruktions-Frames, die ich in den Abschnitten 8.1 bis 8.3 erörtert habe, hängen enger mit den methodologischen Fragen nach möglichen Methoden zur Ermittlung des KonstruktionsFrames zusammen, als es auf den ersten Blick erscheinen mag. Um eine geeignete Methode für eine gegebene Konstruktion zu wählen, ist es von Vorteil, den bevorzugten Mechanismus zur Evokation des Konstruktions-Frames, der dieser Konstruktion zukommt, zu kennen. Die Methoden zur Ermittlung des KonstruktionsFrames hängen also wesentlich mit den Evokationsmechanismen zusammen und können nicht scharf von diesen getrennt werden.

Um diese Abhängigkeit nachzuvollziehen, bietet es sich an, noch einmal das wesentliche Kriterium zur Unterscheidung der verschiedenen Evokationsmechanismen in den Blick zu nehmen: die Frame-Nähe. Aus der Einteilung der Abschnitte 8.2 und 8.3 geht hervor, dass sich die unterschiedlichen Mechanismen zur Evokation des Konstruktions-Frames nach relatierten und unrelatierten lexikalischen Frames unterscheiden lassen (vgl. auch Unterabschnitt 8.1.2). Nach diesen zwei Kategorien lexikalischer Frames lassen sich nun auch die Methoden zur Ermittlung des Konstruktions-Frames unterscheiden. Die beiden äußeren Rechtecke in Abbildung 8.7 sollen diese Unterscheidung verdeutlichen.

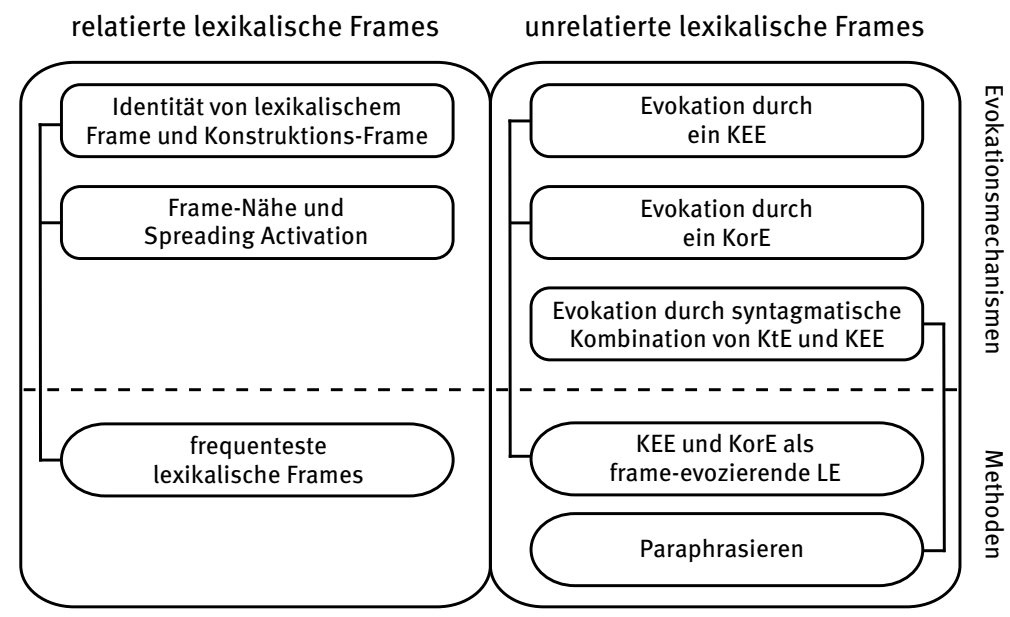

Abb. 8.7: Methoden zur Ermittlung des Konstruktions-Frames nach lexikalischen Frames und Evokationsmechanismen 
Im oberen Teil der beiden Rechtecke sind jeweils die Evokationsmechanismen aufgeführt, die sich nach relatierten und unrelatierten lexikalischen Frames unterscheiden. Im unteren Teil sind dann jeweils die Methoden zur Evokation des Konstruktions-Frames aufgeführt, um zunächst deren Zuordnung nach relatierten und unrelatierten lexikalischen Frames zu verdeutlichen. Der Bezug zwischen den Evokationsmechanismen und den Methoden geht jedoch noch über die Einteilung nach der Frame-Nähe lexikalischer Frames hinaus. So sollen die Verbindungslinien zwischen den Evokationsmechanismen und den Methoden anzeigen, dass zwischen ihnen ein direkter Zusammenhang besteht.

Insgesamt drei Methoden möchte ich in Abschnitt 8.5 diskutieren. Mit lexikalischen Frames operiert die Methode der frequentesten lexikalischen Frames (Unterabschnitt 8.5.1), die den Konstruktions-Frame über relatierte lexikalische Frames rekonstruieren möchte, die von den KtE des KE EREIGNIS der drei untersuchten Konstruktionen evoziert werden. Auf der Seite der relatierten lexikalischen Frames stellt sie die einzige Methode dar und ist insbesondere für die reflexive Bewegungskonstruktion und die reflexive Partikelverbkonstruktion von Relevanz. Diese Methode schließt an zwei Evokationsmechanismen an, die ebenfalls über relatierte lexikalische Frame figurieren: die Identität von lexikalischem Frame und Konstruktions-Frame (Unterabschnitt 8.2.1) sowie die zum Konstruktions-Frame in Frame-Nähe stehenden relatierten lexikalischen Frames und die Prozesse der Spreading Activation (Unterabschnitt 8.2.2). Demgegenüber operieren nun zwei Methoden vorrangig mit unrelatierten lexikalischen Frames. Die Methode der KEE und KorE als frame-evozierende LE (Unterabschnitt 8.5.2) fragt danach, ob jene Strukturelemente (bzw. Teile von ihnen), direkte Hinweise auf den KonstruktionsFrame geben. Sie schließt also direkt an die möglichen Mechanismen der Evokation des Konstruktions-Frames durch ein KEE (Unterabschnitt 8.3.1) bzw. durch ein KorE (Unterabschnitt 8.3.2) an. Diese Methode eignet sich zuvorderst für Konstruktionen, in denen eine Evokation des Konstruktions-Frames über jene Strukturelemente infrage kommt, unter den drei untersuchten Konstruktionen insbesondere die reflexive Weg-Konstruktion. Schließlich arbeitet die Methode des Paraphrasierens (Unterabschnitt 8.5.3) ebenfalls mit unrelatierten lexikalischen Frames und kann besonders dann herangezogen werden, wenn eine Evokation des Konstruktions-Frames durch die syntagmatische Kombination von KtE und KEE (Unterabschnitt 8.3.3) für eine Konstruktion relevant ist. Ebenso wie dieser Mechanismus betrifft sie also in erster Linie die Konstrukte mit unrelatierten lexikalischen Frames der reflexiven Bewegungskonstruktion und der reflexiven Partikelverbkonstruktion.

Die Zusammenhänge zwischen der Unterscheidung lexikalischer Frames nach ihrer Frame-Nähe und den Methoden zur Ermittlung des KonstruktionsFrames mögen nun den Eindruck einer Zirkularität erwecken. Stellt man sich 
eine Ausgangssituation vor, in der der Konstruktions-Frame einer gegebenen Konstruktion noch gänzlich unbekannt ist, ergibt sich deshalb folgender Einwand: Woher kann man die Kategorie der relatierten Frames kennen, wenn man den Konstruktions-Frame nicht kennt? Die Wahl einer Methode zur Ermittlung des Konstruktions-Frames könnte also - so die Schlussfolgerung - nur dann erfolgen, wenn man den Konstruktions-Frame bereits kennt. Ich möchte allerdings zeigen, dass die Gefahr einer solchen Zirkularität wesentlich geringer ist, als es auf den ersten Blick erscheint. Zwei Argumente lassen sich dagegen ins Feld führen.

1. Das System der Frame-Nähen eines Konstruktions-Frames zeigt sich über die exhaustive Annotation der gesamten Treffermenge zur Korpusrecherche einer Konstruktion auf evozierte lexikalische Frames (also sowohl der tatsächlichen Konstrukte als auch der Falschpositive) nahezu automatisch auf.

2. Eine Kombination mehrerer Methoden zur Ermittlung des KonstruktionsFrames kann die Ergebnisse einer Methode, die stark auf der Kategorie der relatierten lexikalischen Frames beruht, absichern, sofern es Methoden gibt, die sich für unrelatierte lexikalische Frames eignen.

Der erste dieser beiden Punkte spricht explizit den methodologischen Gewinn aus der Betrachtung konstruktioneller Ambiguität an, den ich im folgenden Unterabschnitt 8.4.2 thematisieren möchte. Kern dieser Idee ist, dass sich die semantischen Eigenschaften einer Konstruktion und insbesondere - aber nicht nur - der Konstruktions-Frame durch den Vergleich von Falschpositiven einer Korpusrecherche mit den tatsächlichen Konstrukten der betreffenden Konstruktion eruieren lassen. Der zweite der beiden Punkte wird sich bei der eingehenderen Diskussion der Methoden ergeben. Einerseits können die Methoden unabhängig voneinander operieren, je nachdem, ob sie besonders auf Konstrukte mit relatierten oder unrelatierten lexikalischen Frames angewendet werden können, andererseits können sie aber auch komplementär zueinander angewendet werden, insbesondere um die Ergebnisse einer Methode, die auf relatierten lexikalischen Frames beruht, durch eine Methode, die auf unrelatierten lexikalischen Frames beruht, abzusichern. So können die Konstrukte einer Konstruktion gewissermaßen gegenseitig als Testmaterial dienen, um die Adäquatheit einer Analyse, die bereits zu einem potenziellen Konstruktions-Frame als Ergebnis geführt hat, zu überprüfen.

\subsubsection{Konstruktionelle Ambiguität: Warum Falschpositive interessant sind}

Eine der größten Herausforderungen bei der korpusbasierten Analyse einer Konstruktion ist es, unter den Treffern einer Korpusrecherche die Konstrukte dieser 
Konstruktion von irrelevanten Treffern zu trennen, die Konstrukte einer anderer Konstruktion zeigen können. In diesem Arbeitsschritt liegt allerdings nicht nur eine Herausforderung, sondern zugleich die Chance, bereits in diesem frühen Stadium einer Analyse die ,Werte“ wichtiger semantischer Parameter der zu analysierenden Konstruktion zu ermitteln. Dazu gehört in erster Linie das System der Frame-Nähen, das dieser Konstruktion zugeschrieben werden kann, und damit letztendlich auch der Konstruktions-Frame. Auf den Ergebnissen dieses Arbeitsschrittes können deshalb unterschiedlichste Methoden zur Ermittlung des Konstruktions-Frames aufbauen, weshalb gerade die Untersuchung derjenigen Korpustreffer, die keine Konstrukte der betreffenden Konstruktion zeigen, eine wichtige methodische Vorarbeit bei der Ermittlung des Konstruktions-Frames darstellt. Ich möchte in diesem Abschnitt unter Rückgriff auf den Begriff der konstruktionellen Ambiguität und die korpuslinguistische Relevanz von Falschpositiven, also jenen eigentlich irrelevanten Korpustreffern, auf die methodische Relevanz hinweisen, die einer solchen initialen Analyse einer Konstruktion hinsichtlich der Ermittlung des Konstruktions-Frames zukommen kann.

Ein Beispiel: Die beiden Belege (45) und (46) zeigen eine identische formale Struktur. Beide bestehen aus jeweils einer NP, einer VP, einem Reflexivum sowie einer PP im Akkusativ. Nur bei Beleg (45) aber handelt es sich um ein Konstrukt der reflexiven Bewegungskonstruktion. Beleg (46) ist trotz seiner formalen Identität nicht als Konstrukt dieser Konstruktion einzustufen. ${ }^{37}$

(45) Er kämpfte sich durch das Dickicht. (Glavinic, Thomas: Die Arbeit der Nacht, München Wien: Carl Hanser Verlag 2006, S. 273)

(46) Die beklagten Differenzen ergeben sich durch unterschiedliche Rechnungswege. (Die Zeit, 09.03.2000, Nr. 11)

Goldberg (2002) führt für Fälle wie diejenigen in (45) und (46) den Begriff der konstruktionellen Ambiguität ein. Konstruktionelle Ambiguität liegt vor, wenn zwei Instanzen Formgleichheit aufweisen, aber weder eine semantische Ähnlichkeit noch eine semantische Relation zwischen beiden Konstrukten vorliegt: „[T]here do exist instances of constructional ambiguity: a single surface form having unrelated meanings.“ (Goldberg 2002: 335). Bezogen auf die syntaktische Form von Argumentstruktur-Konstruktionen besteht konstruktionelle Ambiguität zwischen zwei Konstrukten in ,shared syntax and completely unrelated function.“ (Goldberg 2009: 208, Anm. 6). Diese nicht vorhandene semantische Relation zwischen

37 Beleg (46) stammt aus den bei der Datenauswahl für die reflexive Bewegungskonstruktion als Falschpositive identifizierten Belegen, die aber, wie bereits in Unterabschnitt 3.4.2 erläutert, nicht ausgesondert, sondern weiter verfügbar gehalten wurden. 
den Konstrukten ist indes das Kriterium, das konstruktionelle Ambiguität von konstruktioneller Polysemie (Abschnitt 5.2) unterscheidet, denn in Letzterer muss zwischen den Konstrukten eine semantische Verbindung bestehen (vgl. Goldberg 1992: 51).

Konstruktionelle (hier also: syntaktische) Ambiguität unterscheidet sich von Ambiguität auf lexikalischer Ebene. Letztere ist wie folgt definiert: „A word or phrase is called to be ambiguous when it has two different meanings, or semantic values.“ (Sweetser 1990: 10). ${ }^{38}$ Im Falle konstruktioneller Ambiguität einer Argumentstruktur-Konstruktion ist es nicht das Konstrukt, das zwei verschiedene semantische Interpretationen besitzt, vielmehr trifft diese Eigenschaft auf die Formseite der Konstruktion zu. Konstrukte, denen eine ambige Formseite zugrunde liegt, sind dagegen semantisch tendenziell eindeutig, die Ambiguität der Formseite ist nur daran zu erkennen, dass Instanzen dieser Formseite als Konstrukte entweder von einer Konstruktion A oder einer Konstruktion B eingestuft werden können, die formseitig gleich sind, sich in ihren semantischen Eigenschaften aber hinreichend unterscheiden. Dies lässt sich an den Belegen (45) und (46) eindeutig sehen: Es fällt nicht schwer, (45) als Konstrukt der reflexiven Bewegungskonstruktion einzustufen und (46) nicht. Dieser Punkt ist also essenziell für die Untersuchung von Korpusdaten: Treffer einer Suchanfrage können entweder als Instanzen der zu untersuchenden Konstruktion identifiziert oder ausgesondert und eventuell als Konstrukte einer anderen Konstruktion eingestuft werden.

Korpuslinguistisch gesehen handelt es sich bei Belegen wie (46), die bei der Korpusrecherche für eine Konstruktion wie die reflexive Bewegungskonstruktion mehr oder weniger zwangsläufig auftreten, um Falschpositive (false positives): Es sind „hits that are not instances of our phenomenon“ (Stefanowitsch 2020: 111). Genauer: Es sind Treffer, die zwar der Form der zu untersuchenden Konstruktion, also etwa der reflexiven Bewegungskonstruktion, entsprechen, aber semantisch deutlich von ihr abweichen und im Sinne der Definition konstruktioneller Ambiguität von Goldberg (2002: 335) auch keine semantische Relation zu der untersuchten Konstruktion aufweisen. ${ }^{39}$ Dadurch, dass sich Falschpositive nicht for-

38 Hier ist noch einmal auf die Unterscheidung zwischen (konstruktioneller) Polysemie und Ambiguität hinzuweisen, die Goldberg (1992: 51, 2002: 335, 2009: 208, Anm. 6) auflöst, indem sie davon ausgeht, dass (konstruktionelle) Polysemie auf einer Verbindung mehrerer Lesarten beruht, während diese Verbindung für Ambiguität gerade nicht gegeben ist.

39 Wie bereits in Unterabschnitt 3.4.2 angedeutet, betrachte ich als Falschpositive also lediglich solche Belege, die formal identisch zu den drei untersuchten Konstruktionen sind, aber sich semantisch von ihnen unterscheiden. Diese bleiben deshalb in der Datenauswahl. Belege, die sich formal und semantisch unterscheiden, werden gänzlich ausgesondert und spielen für die Überlegungen in diesem Unterabschnitt keine Rolle. 
mal, sondern nur semantisch von den Konstrukten einer zu untersuchenden Konstruktion unterscheiden, eignet sich ihre Betrachtung besonders, um die semantischen Eigenschaften jener Konstruktion zu eruieren und sie von anderen Konstruktionen abzugrenzen. Es ist deshalb sinnvoll, solche Falschpositive, wie ich es getan habe (vgl. Unterabschnitt 3.4.2), in der Datenauswahl zu einer Konstruktion zu behalten (aber entsprechend zu markieren), um sie weiterhin auswerten zu können. Falschpositive sind somit keineswegs ,Datenmüll‘, sondern sie lassen sich methodologisch im Hinblick auf die Ermittlung der semantischen Eigenschaften der Konstruktion und insbesondere des Konstruktions-Frames nutzbar machen.

Die Analyse von Falschpositiven kann nun die Grundlage dafür schaffen, Methoden zur Ermittlung des Konstruktions-Frames anwenden zu können - insbesondere solche, die gemäß der Unterscheidung in Unterabschnitt 8.4.1 auf relatierten lexikalischen Frames beruhen. Die Rolle lexikalischer Frames ist hier also von besonderer Relevanz. Wie kann eine solche Analyse aussehen? Folgende drei Schritte in der Annotation der Daten sind dafür notwendig.

1. Alle Treffer der Korpusrecherche müssen, soweit wie möglich, exhaustiv auf ihre lexikalischen Frames hin annotiert werden (soweit sich hierfür Frames in FrameNet finden lassen).

2. Ein Cluster lexikalischer Frames, die zueinander in Frame-zu-Frame-Relationen (auch über mehrere Hierarchieebenen hinweg) stehen, muss identifiziert werden.

3. Für mindestens dieses Cluster, idealerweise aber für alle Treffer, muss eine Annotation der semantischen Motivierung der einzelnen Strukturelemente der Instanzen durch FE vorgenommen werden.

Die ersten beiden dieser Schritte sind Voraussetzung dafür, die relatierten lexikalischen Frames und den Konstruktions-Frame identifizieren zu können. Der dritte Schritt dient insbesondere der Ermittlung unrelatierter lexikalischer Frames, gleichzeitig aber auch zur Absicherung der Ergebnisse zu den relatierten lexikalischen Frames. Der erste Schritt wiederum ist Voraussetzung für den zweiten Schritt. Eine exhaustive Annotation aller Belege auf ihre lexikalischen Frames ermöglicht es, diese lexikalischen Frames daraufhin zu untersuchen, ob sie Cluster bilden, die als Systeme von Frame-Nähen eines potenziellen KonstruktionsFrames infrage kommen. Dies ist am einfachsten über eine entsprechende Visualisierung aller annotierten lexikalischen Frames und deren Frame-zu-FrameRelationen möglich, die auch in ein konstruktikographisches Analysesystem 
Tab. 8.6: Korpusbelege der reflexiven Bewegungskonstruktion mit einem Cluster potenzieller relatierter lexikalischer Frames

\begin{tabular}{lrr}
\hline & Belege & Anteil \\
\hline Belege mit einem Cluster lexikalischer Frames & 712 & $13,76 \%$ \\
Belege mit Anteil eines potenziellen Konstruktions-Frames & 299 & $5,78 \%$ \\
Belege mit anderen lexikalischen Frames & 4.162 & $80,46 \%$ \\
\hline Ausgewählte Belege gesamt & 5.173 & $100,00 \%$ \\
\hline
\end{tabular}

Tab. 8.7: Korpusbelege der reflexiven Partikelverbkonstruktion mit einem Cluster potenzieller relatierter lexikalischer Frames

\begin{tabular}{lrr}
\hline & Belege & Anteil \\
\hline Belege mit einem Cluster lexikalischer Frames & 304 & $8,52 \%$ \\
Belege mit Anteil eines potenziellen Konstruktions-Frames & 546 & $15,30 \%$ \\
Belege mit anderen lexikalischen Frames & 2.719 & $76,18 \%$ \\
\hline Ausgewählte Belege gesamt & 3.569 & $100,00 \%$ \\
\hline
\end{tabular}

implementiert werden kann. ${ }^{40}$ Für die drei untersuchten Konstruktionen möchte ich dies allerdings lediglich exemplarisch an den quantitativen Verhältnissen der Korpusbelege aufzeigen.

In den Tabellen 8.6 und 8.7 sind für die reflexive Bewegungskonstruktion und die reflexive Partikelverbkonstruktion die Verhältnisse von Belegen angegeben, die ein Cluster potenziell relatierter lexikalischer Frames (inklusive des Konstruktions-Frames) bilden, zu Belegen, die potenzielle unrelatierte lexikalische Frames mit einem Anteil eines Konstruktions-Frames beinhalten, sowie allen weiteren Belege von Falschpositiven mit anderen (ggf. andere Cluster bildenden) lexikalischen Frames..$^{41}$ Für die reflexive Weg-Konstruktion können diese Verhältnisse nicht angegeben werden, da sich, wie in Unterabschnitt 3.4.2 erläutert, ihre Daten zum Teil aus Ergebnissen der Korpusrecherche zur reflexiven Bewegungskonstruktion zusammensetzen. Aufgrund der ohnehin sehr geringen Datenmenge zur reflexiven Weg-Konstruktion kann sie deshalb für die folgenden Überlegungen ausgeklammert werden.

40 Für die konstruktikographische Arbeit sind Falschpositive auch deshalb besonders interessant, weil sie schon aus computerlinguistischer Sicht Auskunft über die Schwächen eines Ansatzes geben (vgl. Ehrlemark, Johansson \& Lyngfelt 2016: 821).

41 Vgl. Unterabschnitt 3.4.2 für die Zusammensetzung der Daten. 
Aus den Tabellen geht hervor, dass 13,76 \% der ausgewählten Belege für die reflexive Bewegungskonstruktion und 8,52 \% derjenigen für die reflexive Partikelverbkonstruktion ein Cluster lexikalischer Frames bilden, das sich als (Ausschnitt aus dem) System der Frame-Nähen von Motion herausstellt. Allein durch diese Analyse lexikalischer Frames lassen sich zwei wesentliche Beobachtungen machen, die die konstruktionelle Ambiguität der Formseiten der beiden Konstruktionen aufzulösen imstande sind.

- Diejenigen Belege, die dem Cluster lexikalischer Frames angehören, weisen semantische Beziehungen zueinander auf und kommen somit als Konstrukte einer gemeinsamen Konstruktion infrage.

- Diese Belege sind von denjenigen mit anderen lexikalischen Frames (dritte Zeile in den Tabellen 8.6 und 8.7) und solche mit einem Anteil eines potenziellen Konstruktions-Frames (zweite Zeile) hinreichend abgegrenzt, sodass Erstere eindeutig als Falschpositive identifiziert werden können.

Wenn sich, wie für die reflexive Bewegungskonstruktion und die reflexive Partikelverbkonstruktion, bestätigt, dass das Cluster lexikalischer Frames als System der Frame-Nähe eines potenziellen Konstruktions-Frames infrage kommt, sind damit bereits alle belegten relatierten lexikalischen Frames identifiziert. Sie müssen nicht zwangsläufig mit dem gesamten System der Frame-Nähen des Konstruktions-Frames identisch sein, da dazu auch relatierte lexikalische Frames gehören können, die nicht belegt sind (vgl. die Tabellen 5.12 bis 5.17 in Unterabschnitt 5.4.3). Über die Frame-zu-Frame-Relationen in FrameNet können diese jedoch einfach ergänzt werden, womit sich das System der Frame-Nähen des potenziellen Konstruktions-Frames rekonstruieren lässt.

Hinzu kommen nun noch die Konstrukte, deren Konstrukt-Frames mit unrelatierten lexikalischen Frames und dem Anteil eines Konstruktions-Frames konstituiert werden, bei denen also eine Frame-Anpassung des lexikalischen Frames im Konstrukt-Frame stattfindet. Diese können in der Annotation dadurch identifiziert werden, dass die semantische Motivierung ihrer Strukturelemente nicht vollständig durch FE des lexikalischen Frames geleistet werden kann, bei einem potenziellen KE oder KEE also entweder eine einfache Motivierung durch einen potenziellen Konstruktions-Frame (Unterabschnitte 6.2.2 und 6.3.2) oder eine doppelte Motivierung von FE des lexikalischen Frames und eines KonstruktionsFrames (Unterabschnitte 6.2.3 und 6.3.3) vorliegt. An den beiden oben als (45) und (46) zitierten Belegen lässt sich dies nachvollziehen. An Beleg (45), den ich hier als (47) wiederhole, lässt sich erkennen, dass eine semantische Motivierung des Reflexivums sowie der PP nicht durch den lexikalischen Frame Hostile_encounter, der durch die LE kämpfen (fight.v) evoziert wird, gewährleistet werden kann (hier durch Fragezeichen angedeutet). 
[SIDE_1 Er] [Hostile_encounter kämpfte] [sich ? ] [durch das Dickicht ${ }^{\text {? }}$ ]. (Glavinic, Thomas: Die Arbeit der Nacht, München Wien: Carl Hanser Verlag 2006, S. 273)

Bei Beleg (46), den ich hier als (48) wiederhole, lässt sich diese Situation nicht beobachten. Der Frame Causation, den die LE ergeben (result.v) evoziert, zählt nicht zum ermittelten Cluster lexikalischer Frames und ist gleichzeitig in der Lage, alle Strukturelemente des Konstrukts zu motivieren. ${ }^{42}$ Dieser Beleg kann daher als Falschpositiv identifiziert werden, während derjenige in (47) als relevantes Konstrukt identifiziert werden kann. Der Frame Hostile_encounter kann damit als unrelatierter lexikalischer Frame gelten, der Frame Causation nicht.

[EFFEct Die beklagten Differenzen] [causation ergeben] sich [CAuse durch unterschiedliche Rechnungswege]. (Die Zeit, 09.03.2000, Nr. 11)

Diese Ergebnisse zeigen, dass die reine Analyse der Formseite einer Konstruktion kein hinreichendes Mittel ist, um ihre Konstrukte zu identifizieren und Instanzen, die nicht als ihre Konstrukte anzusehen sind, auszuschließen. Verhagen (2009) beobachtet Ähnliches für die Ditransitivkonstruktion und argumentiert, dass eine das Gewicht auf die Formseite legende Analyse zeichentheoretisch inadäquat ist:

Could it also be a matter of convention that three participant events are events of transfer? In other words: Is it also a rule (of English) to use a three participant frame in this way, i.e. essentially in the same way as it is a matter of convention that the sound shape give is a signal for events of transfer? Minimally, I would say that this would then come on top of the 'motivatedness' of the construction in terms of causal knowledge. Moreover, it is certainly not the case that the presence of three nominals (i.e. the recognition of three different expressions as belonging to the class of noun phrases) conventionally signifies that we have to think of an event of transfer. (Verhagen 2009: 137)

Die reflexive Bewegungskonstruktion und die reflexive Partikelverbkonstruktion sind anschauliche Beispiele dafür, dass die Beschreibung einer Konstruktion, die etwa die Benennung und Definition ihrer Strukturelemente umfasst (Abschnitt 7.3), wesentlich von einer frame-semantischen Annotation profitieren kann. Diese hat nicht nur konstruktionssemantische und konstruktikographische Relevanz, sondern kann bei der initialen Entscheidung helfen, ob ein Beleg ein Konstrukt dieser Konstruktion zeigt oder nicht (vgl. dazu schon Unterabschnitt 3.4.2).

42 Das Reflexivum fällt hier heraus, da die LE ergeben (result.v) als ,echt' reflexives Verb angesehen werden kann und es sich bei Causation um einen rezessiven lexikalischen Frame handelt, der kein FE für das Reflexivum bereitstellt (vgl. zu dieser Problematik Unterabschnitt 6.3.1). 
Die frame-semantische Annotation und der Vergleich zwischen denjenigen Korpustreffern, die sich als Konstrukte der Konstruktion beinhaltend herausstellen, mit den Falschpositiven, bieten die Chance, semantische Eigenschaften der Konstruktion bereits in diesem frühen Stadium der Analyse zu identifizieren und sie von anderen Konstruktionen abzugrenzen. Sie sind nicht nur Voraussetzung für die Ermittlung des Konstruktions-Frames, sondern gleichermaßen unter anderem für die Beschreibung von Präferenzen und Beschränkungen der einzelnen Strukturelemente der Konstruktion (Abschnitt 5.3) sowie deren Benennung (Abschnitt 7.3). Zum Aspekt der Präferenzen schreibt Verhagen (2009) weiter:

It is knowledge of this relationship that precisely allows class membership to function as a trigger (together with other triggers in typical cases) for a typical environment in which it may occur, i.e. to function as an aspect of the form of a construction. Thus, what is meant by saying that class membership can function as an aspect of form and have a symbolizing function, is that the recognition of a particular element as belonging to the class that fits a particular 'slot' of a construction [d.h. die Erfüllung der Präferenz z.B. eines KE, A.W.] contributes to the recognition of the construction. In particular, an element's belonging to a class defined by a slot of a construction [eine Präferenz, A.W.] may at least be taken as a (weaker or stronger) symptom of the presence of the construction, [...]. (Verhagen 2009: 140).

Das Klassifizieren der Korpustreffer in (potenzielle) Konstrukte der zu untersuchenden Konstruktion und Falschpositive ist somit ein entscheidender Schritt, sich dem ,Wert' des semantischen Parameters der Frame-Nähe (Abschnitt 5.4) und damit den semantischen Eigenschaften der Konstruktion, insbesondere ihrem Konstruktions-Frame, und letztlich ihrer konstruktikographischen Beschreibung zu nähern.

Mit dieser Analyse von konstruktioneller Ambiguität ist also das potenzielle System der Frame-Nähen einer Konstruktion identifiziert. Ein KonstruktionsFrame wurde damit aber noch immer nicht festgelegt. An dieser Stelle treten die dezidierten Methoden zu seiner Ermittlung auf den Plan.

\subsection{Methoden zur Ermittlung des Konstruktions-Frames}

Dass der Konstruktions-Frame der reflexiven Bewegungskonstruktion, der reflexiven Partikelverbkonstruktion und der reflexiven Weg-Konstruktion mit Motion zu identifizieren ist, habe ich über den gesamten bisherigen Verlauf dieser Arbeit als gegeben vorausgesetzt. Begründet habe ich die Entscheidung für genau diesen Frame noch nicht. Das Streben nach einer solchen Begründung wirft ein Problem auf, das eigentlich am Beginn der konstruktionssemantischen Analyse zu erwarten wäre: Wie kann der Konstruktions-Frame einer gegebenen Konstrukti- 
on methodisch ermittelt werden? Methodische Fragen, die die semantischen Eigenschaften von Konstruktionen betreffen, stellen sich indes nicht nur in Analysen, die sich ihnen dezidiert frame-semantisch nähern, sondern grundsätzlich in jedem Ansatz, der semantische Aspekte von Konstruktionen zu explizieren versucht: ${ }^{43}$ „An important question that a constructional approach to argument structure must address is how syntactic patterns come to be associated with meaning." (Perek 2015: 80).

Über solche methodischen Fragen wird sowohl in der gebrauchsbasierten Konstruktionsgrammatik als auch in der Konstruktikographie jedoch kaum reflektiert. Eine intersubjektive Plausibilität der Analysen erscheint oft als gegeben vorausgesetzt, ohne dass expliziert wird, wie es etwa zu der Wahl eines bestimmten Frames als Konstruktions-Frame kommt. In diesem Sinne bemerkt Dewell (2011), dass die Analyse der semantischen Eigenschaften einer Konstruktion bislang unter dem Defizit einer Subjektivität steht, was eine Ursache dafür sein könnte, sie gänzlich zu unterlassen:

\begin{abstract}
One problem with positing subjective construal processes such as perspectival modes [Dewells Ansatz für Partikel- und Partikelpräfixverben, A.W.] as the meaning of a grammatical construction is that this kind of meaning is more difficult to prove than normal lexical meaning would be. It does not lend itself to the usual accepted types of argumentation, which is a major reason why linguists tend to avoid the whole topic of constructional meaning. Many skeptical readers will no doubt read the hypotheses stated above [seine Annahmen für die semantischen Eigenschaften von Partikel- und Partikelpräfixverben, A.W.] and find them too subjective to be demonstrated in a scientific way. (Dewell 2011: 17)
\end{abstract}

Es ist klar, dass ein konstruktionssemantischer Ansatz vor diesem Hintergrund die Frage danach, welcher Frame als Konstruktions-Frame einer gegebenen Konstruktion anzusehen ist, nicht außer Acht lassen darf. Ursächlich dafür ist nach derzeitigem Stand insbesondere der konstruktikographischen Forschung die von Dewell (2011: 17) angedeutete Frage nach möglichen Unterschieden zwischen den semantischen Eigenschaften syntaktischer Konstruktionen und denjenigen einfacher LE, ${ }^{44}$ die sich in einer Asymmetrie semantischer Ressourcen für die Analyse von Konstruktionen widerspiegelt: Während mit FrameNet eine vergleichsweise große Datenbasis für LE existiert, die zumindest als Ausgangspunkt zur Beantwor-

43 Vgl. Finkbeiner (2008: 125-127) für ähnliche Herausforderungen und einige Lösungsvorschläge bei der Analyse der semantischen Eigenschaften bestimmter Phraseme.

44 Vgl. dazu weiter: „We are also not in the habit of distinguishing the semantic contribution of the grammatical construction itself from the contribution of its more particular components, and we are much more consciously aware of choosing individual lexemes than we are of choosing a grammatical pattern. Introspection in fact leads some to conclude that there is no such thing as the meaning of a grammatical construction.“ (Dewell 2011: 17). 
tung der Frage, welche Frames bestimmte LE (potenziell) evozieren, dienen kann, existiert eine genuin für syntaktische Konstruktionen entwickelte Datenbasis von Frames bislang nicht (vgl. Unterabschnitt 2.1.3 sowie Abschnitt 8.1). Mit anderen Worten: Für LE lässt sich derzeit ,nachschlagen', welche Frames sie evozieren, für syntaktische Konstruktionen hingegen (noch) nicht. Dass dieser Punkt nicht trivial ist, scheint in der bisherigen Forschung, die Frames für die semantischen Eigenschaften von Konstruktionen heranzieht, kaum wahrgenommen zu werden. Dies führt bisweilen zu Kuriositäten der Annahme scheinbar kanonisierter Forschungsergebnisse, die jedoch alles andere als methodisch nachvollziehbar sind. Ein Beispiel dafür ist die folgende Darstellung von Hasegawa et al. (2010), die direkt an die von ihnen geäußerte Annahme, dass Konstruktionen Frames evozieren können, anschließt:

The constructional evocation of frames was explored in detail by Goldberg (1995), who demonstrated that the Ditransitive construction (V NP NP, slide her the papers) had the semantics of the Cause_receive frame (Chapter 6), and that of the make one's way construction (whistled her way down the street) evokes the Motion frame (Chapter 9). (Hasegawa et al. 2010: 171)

Die Frames, die Hasegawa et al. (2010) gewissermaßen als Konstruktions-Frames annehmen, sind unverkennbar FrameNet-Frames..$^{45}$ Die Erkenntnis über diese Frames schreiben sie Goldberg (1995) zu - erkennbar an den Verweisen auf die entsprechenden Kapitel bei Goldberg. Allerdings existierte FrameNet bei dem Erscheinen von Goldbergs Studie noch gar nicht. ${ }^{46}$ Statt die Auswahl dieser Frames selbst zu begründen, wird deren Annahme einer Studie zugeschrieben, die diese Erkenntnis gar nicht hervorgebracht haben kann und zudem, wie ich in Unterabschnitt 2.2.2 gezeigt habe, auf ein ganz anderes Frame-Modell, nämlich die Kasusgrammatik (Unterabschnitt 2.1.1), zurückgreift.

Ohne dieses Einzelproblem weiter kritisieren zu wollen, beginnt die Suche nach der Quelle für die Annahme eines bestimmten Frames als KonstruktionsFrame zunächst verständlicherweise bei dem Forschungsstand zu der betreffenden Konstruktion. So beruht die wesentliche Motivation, Motion zur konstruktionssemantischen Analyse der reflexiven Bewegungskonstruktion, der reflexiven Partikelverbkonstruktion und der reflexiven Weg-Konstruktion heranzuziehen, zunächst auf vorgängigen Analysen, insbesondere im Rahmen der FrameNet-Konstruktikographie, die für die way-Konstruktion auf ebendiesen Fra-

45 Der Frame Cause_receive existiert im FrameNet-Release 1.7 allerdings unter diesem Namen nicht (mehr). Er könnte entweder in Transfer oder Receiving aufgegangen sein, wobei Letzterer Transfer perspektiviert, ihm also in der Perspektive_auf-Relation untergeordnet ist.

46 Die Arbeiten an FrameNet begannen im Jahr 1997 (vgl. Ruppenhofer, Boas \& Baker 2018: 477). 
me (wie Fillmore, Lee-Goldman \& Rhomieux 2012: 322) oder Self_motion (wie Lee-Goldman \& Petruck 2018: 32-33), der zu Motion in der Vererbungsrelation untergeordnet ist, zurückgegriffen haben (vgl. Unterabschnitt 2.3.2). Eine genuine konstruktionssemantische Analyse kann sich auf eine solche überindividuelle Plausibilität allerdings nicht verlassen, zumal der konstruktikographische Forschungsstand nicht für jede Konstruktion derart umfangreich ist wie für die way-Konstruktion, um ihn auf ihre deutschen Äquivalente zu übertragen. Wichtig wird die Frage nach Methoden zur Ermittlung des Konstruktions-Frames besonders also bei Analysen, die nicht auf vorgängige Evidenz zurückgreifen können.

Zum Abschluss dieses Kapitels möchte ich nun jene methodischen Aspekte in den Fokus rücken, die der Beantwortung der Frage nach dem KonstruktionsFrame einer gegebenen Konstruktion auf eine objektivere Basis stellen können. Für sie gilt zunächst dasselbe wie für die in den Abschnitten 8.2 und 8.3 diskutierten Mechanismen der Evokation des Konstruktions-Frames: Sie beanspruchen ihre Geltung in erster Linie für die drei untersuchten Konstruktionen. Noch wichtiger als für die Frage nach der Evokation des Konstruktions-Frames ist für dessen methodische Ermittlung aber hervorzuheben, dass die in diesem Abschnitt diskutierten Methoden auch auf andere Konstruktionen Anwendung finden können. Insbesondere aus konstruktikographischer Hinsicht ist die Ermittlung des Konstruktions-Frames indes relevanter als Überlegungen zu dessen Evokation, da die Angabe des Konstruktions-Frames ein elementarer Bestandteil eines Konstruktionseintrags ist (vgl. Unterabschnitt 7.1.3).

Nachdem ich in Abschnitt 8.4 die Präliminarien zur Anwendung einiger Methoden zur Ermittlung des Konstruktions-Frames diskutiert habe, sollen in diesem Abschnitt die drei konkreten Methoden, die ich bereits in Unterabschnitt 8.4.1 erwähnt habe, vorgestellt werden. Ich beginne in Unterabschnitt 8.5.1 mit der Methode der frequentesten lexikalischen Frames, die ich vor allem auf die reflexive Bewegungskonstruktion und die reflexive Partikelverbkonstruktion und deren Konstrukte mit relatierten lexikalischen Frames anwende. In Unterabschnitt 8.5.2 stehen noch einmal KEE und KorE im Vordergrund und die Frage, ob sie bzw. Teile von ihnen als den Konstruktions-Frame evozierende LE infrage kommen. Unter den drei untersuchten Konstruktionen bietet sich für eine solche Analyse die reflexive Weg-Konstruktion an. Schließlich soll in Unterabschnitt 8.5.3 eine Methode vorgestellt werden, die für Konstrukte aller drei Konstruktionen mit unrelatierten lexikalischen Frames herangezogen werden kann und die auf dem Paraphrasieren dieser Konstrukte basiert.

Trotz der Tatsache, dass sich die im Folgenden diskutierten Methoden an den drei untersuchten Konstruktionen nicht im gleichen Maße aufzeigen lassen - so kann die mit KEE und KorE arbeitende Methode vor allem an der reflexiven WegKonstruktion aufgezeigt werden -, ist zu betonen, dass die Wahl der Methode 
nicht grundsätzlich eine Entweder-Oder-Entscheidung ist, die sich nach der gegebenen Konstruktion richten muss. Vielmehr können für dieselbe Konstruktion auch mehrere Methoden zur Ermittlung des Konstruktions-Frames angewendet werden: Komplementär sind insbesondere Methoden, die mit relatierten lexikalischen Frames operieren, mit solchen, die mit unrelatierten lexikalischen Frames operieren. Aus diesem Grund empfiehlt es sich, für eine Anwendung auf weitere Konstruktionen stets mehrere Methoden an einer Konstruktion zu erproben, um diejenige Methode zu ermitteln, die für die betreffende Konstruktion am geeignetsten erscheint.

\subsubsection{Frequenteste lexikalische Frames}

In der gebrauchsbasierten Konstruktionsgrammatik wird des Öfteren davon ausgegangen, dass sich die semantischen Eigenschaften einer Konstruktion über diejenigen der LE, die als KtE in ihre KE eintreten können, rekonstruierbar ist. ${ }^{47}$ Bei Argumentstruktur-Konstruktionen betrifft dies in erster Linie dasjenige KE, das Verben als KtE aufweist. Perek (2015) bezeichnet diese Annahme als lexical origin hypothesis und fasst sie wie folgt zusammen: 48

A large body of evidence suggests that there is a close connection between the meaning of an argument structure construction and the usage of this construction, particularly as it pertains to the verbs occurring in it. Since the occurrence of a verb in a construction is governed by principles of semantic compatibility, the existence of such a connection is not particularly surprising. The observation of the verbs that occur in a construction is expected to provide information as to the construction's meaning, for speakers as well as for linguists. (Perek 2015: 80)

Motiviert ist diese Einsicht durch das methodische Instrument der Kollostruktionsanalyse (Stefanowitsch \& Gries 2003, 2005; Gries \& Stefanowitsch 2004a,b;

47 Willems \& Coene (2006: 263) weisen darauf hin, dass dies im Gegensatz zu der Auffassung von ,grammatischen' Bedeutungen von Konstruktionen steht, die, wie in Unterabschnitt 4.3.3 gesehen, bisweilen auch in der Konstruktionsgrammatik angenommen werden - was Willems \& Coene (2006: 263) allerdings leugnen.

48 Eine entsprechende theoretische Annahme findet sich bereits bei Goldberg (1999: 202). Empirische Evidenz liefern etwa Goldberg, Casenhiser \& Sethuraman (2003, 2004). Für ein Beispiel der Ditransitivkonstruktion vgl. Croft (2012: 381): „Any meaning attributed to the Ditransitive construction is an abstraction across the range of verbs that occur in it.“ Hilpert (2012: 234) überträgt die entsprechende Idee in eine diachrone Perspektive, nach der der semantische Wandel einer Konstruktion mit demjenigen der LE, die in sie eintreten können, einhergeht und auch durch neu hinzukommende LE ausgelöst wird. 
Stefanowitsch 2013), die darauf abzielt, die statistische Signifikanz der KtE eines KE (in dortiger Terminologie: dessen Kollexeme) an die Konstruktion zu messen. Die semantischen Eigenschaften eines besonders stark mit einer Konstruktion assoziierten Kollexems sollten sich dann also auf die semantischen Eigenschaften der Konstruktion als Ganzes übertragen lassen. ${ }^{49}$ Die Kollostruktionsanalyse argumentiert also konkret auf der Basis von Präferenzen (Abschnitt 5.3), genauer: den lexikalischen Präferenzen bei der Instanziierung eines oder mehrerer KE. Die Untersuchung solcher Präferenzen oder Beschränkungen auf lexikalischer Ebene hat in der Konstruktionsgrammatik eine lange Tradition. Schon Fillmore (1989) gibt zu bedenken:

Each grammatical construction can be identified with a certain set of components, a collection of constraints on the syntax or semantics of the components, a statement of the use of the construct as a whole, together with a set of instructions for incorporating information linked with its parts into an interpretation of the whole. The constraints will include conditions on the thematic or categorial identity of the components, requirements on the morphological or lexical tagging or heading of specific components, and the linear order of elements where this is relevant. (Fillmore 1989: 19)

Auch Zwicky (1994: 617) spitzt eine solche lexikalische Argumentation zu: „[F]or each formal condition that mentions a slot filled by a word, there is a special set of lexical items eligible to occur in that slot." Obwohl mittlerweile durchaus ebenso gut dokumentiert ist, dass „Beschränkungen (constraints) [...] sowohl semantischer als auch grammatischer Natur sein“ (Ziem 2018e: 30) können, ist ein konsequenter Einbezug semantischer Faktoren gerade ausgehend von Entwicklungen in der methodischen Ermittlung solcher Beschränkungen und Präferenzen durch die Kollostruktionsanalyse allerdings noch eher selten.

So ist dieses zunächst lediglich auf Kollexemen, also einzelnen LE beruhende Verfahren insbesondere im Hinblick auf semantische Fragestellungen vereinzelt kritisiert worden. Beispielsweise argumentiert Bybee (2010: 98), dass „[p]roponents of Collostructional Analysis hope to arrive at a semantic analysis, but do not include any semantic factors in their method. Since no semantic considerations go into the analysis, it seems plausible that no semantic analysis can emerge from it.“ Allerdings zeigt Gries (2012: 492-496) in seiner Replik

49 Als Reflex der Popularität der Kollostruktionsanalyse sind die Vorschläge von Herbst (2016: 180-184, 2018a,b) zu deuten, Informationen zu den Kollexemen einer Konstruktion in die konstruktikographische Beschreibung im Sinne eines Kollostruktikons zu integrieren. Allerdings halte ich es aus konstruktionssemantischer Sicht für angebrachter, den semantischen Informationen in Gestalt von Frames Priorität gegenüber rein lexikalisch basierten und damit zunächst semantikfreien Informationen über die lexikalischen Präferenzen einer Konstruktion einzuräumen. 
auf Bybee, dass die Kollostruktionsanalyse sehr wohl semantische Aspekte integrieren kann, was im Übrigen bereits der Überblick über die Bezugnahme auf Frames in entsprechenden Arbeiten gezeigt hat (Unterabschnitt 2.2.3). Bemerkenswert ist vor diesem Hintergrund, dass bereits Kollostruktionsanalysen für die way-Konstruktion vorliegen. So finden Gries \& Stefanowitsch (2010: 84-86) auf der Grundlage einer kovariierenden Kollexemanalyse (Gries \& Stefanowitsch 2004a; Stefanowitsch \& Gries 2005), die den Slot für das Verb mit demjenigen für die PP in Beziehung setzt, drei semantische Cluster von Verben, unter denen sich interessanterweise ein wiederum in sich unterteilbares Cluster von ,Bewegungsverben' findet, von welchem aus sich Rückschlüsse auf die semantischen Eigenschaften der Konstruktion ableiten lassen dürften. ${ }^{50}$ Dass lexikalische Präferenzen grundsätzlich mit den semantischen Eigenschaften der Konstruktion korrelieren dürften, bemerkt für die way-Konstruktion indes bereits Goldberg (1995: 199-200), allerdings untersucht sie solche Präferenzen nicht systematisch. Wenngleich sich dies für die englische Konstruktion etwa durch die Studie von Gries \& Stefanowitsch (2010: 84-86) mittlerweile geändert hat, liegen vergleichbare Ergebnisse für das Deutsche, also für die reflexive Bewegungskonstruktion oder eine ihrer verwandten Konstruktionen, bisher nicht vor. ${ }^{51}$

Anstatt nun eine kovariierende Kollexemanalyse für die drei untersuchten Konstruktionen durchzuführen (etwa für die KtE des KE EREIGNIS und die präpositionalen Köpfe der KtE des KE WEG der reflexiven Bewegungskonstruktion oder die Instanzen des KEE RICHTUNG der reflexiven Partikelverbkonstruktion), um aus dieser letztendlich den Konstruktions-Frame abzuleiten, möchte ich aus genuin konstruktionssemantischer Perspektive ein anderes Verfahren vorschlagen. Zur Begründung sei ein letzter Blick auf eine potenzielle Gefahr einer Kollostruktionsanalyse gerichtet. Perek (2015: 90-102) findet in einer Fallstudie zur englischen Konativkonstruktion, die ihmzufolge über stark abstrakte semantische Eigenschaften verfügt, unter den am stärksten assoziierten Kollexemen eine Vielzahl semantisch heterogener LE, von denen keine für eine Entsprechung ihrer se-

50 Vgl. dazu auch die Ergebnisse von Stefanowitsch \& Gries (2005: 18-22), ebenfalls zur wayKonstruktion. Sie stellen fest, dass ,the twelve prepositions discussed here provide overwhelming evidence for the fact that verb-preposition pairs in the way-construction display imageschematic coherence.“ (Stefanowitsch \& Gries 2005: 22). Auch dort werden also, entgegen der Kritik von Bybee (2010: 98), genuin semantische Aspekte berücksichtigt.

51 Die Studie von Goschler \& Stefanowitsch (2010) nimmt zwar eine kovarrierende Kollexemanalyse für die Assoziationen zwischen einzelnen ,Bewegungsverben` und Verbpartikeln vor, die grundsätzlich also auch für die reflexive Partikelverbkonstruktion einschlägig wäre, allerdings beschränkt sich ihre Analyse gerade auf ,Bewegungsverben` und geht nicht auf das mögliche Auftreten eines Reflexivums ein. 
mantischen Eigenschaften mit denen der Konstruktion eindeutig infrage kommt. Die rein lexikalische Rekonstruktion der semantischen Eigenschaften einer Konstruktion, die über einzelne LE figuriert, ist deshalb nicht ausreichend:

\begin{abstract}
In conclusion, the case of the conative construction conflicts with the lexical origin hypothesis, in that there is a mismatch between the verbs that the construction most strongly attracts and the central meaning of the construction, which can only be derived from verbs which figure less prominently among the construction's collexemes. This case study shows that the lexical origin hypothesis in its present formulation is an incomplete proposal. It does not tell the whole story about the emergence of constructional meaning: while it provides an explanation for the facilitating effect of verb biases and makes accurate predictions for many constructions, it is by itself insufficient. (Perek 2015: 102)
\end{abstract}

Als Lösung für dieses Problem verändert Perek (2015: 111-142) den Blickwinkel von einer Kollostruktionsanalyse, die über sämtliche für die Konstruktion belegten Kollexeme und damit einer ungeordneten Menge an Konstrukten operiert, hin zu mehreren getrennten Analysen, die auf einem niedrigeren Abstraktionsgrad angesiedelt sind und vorab eine semantische Klassifikation der Kollexeme und damit der Konstrukte der Konstruktion in einzelne Verbklassen vornehmen. Aus konstruktionssemantischer Sicht können statt solcher Verbklassen schlicht Frames verwendet werden, genauer: lexikalische Frames. ${ }^{52}$ Ausgehend von der Hypothese, dass sich die semantischen Eigenschaften einer Konstruktion aus denjenigen besonders signifikanter Kollexeme ableiten lassen, sollte nun also erwartbar sein, dass sich der Konstruktions-Frames unter den besonders frequenten lexikalischen Frames, die für eine Konstruktion belegt sind, wiederfinden lässt und im Idealfall sogar mit dem frequentesten lexikalischen Frame identisch ist. Es gilt nun, diese Hypothese empirisch zu testen und $\mathrm{zu}$ hinterfragen, wie zuverlässig eine solche Analyse als Methode zur Ermittlung des Konstruktions-Frames ist.

Warum lexikalische Frames? Abgesehen davon, dass sich von ihnen direkter auf einen Konstruktions-Frame schließen lässt, da eine Identität von lexikalischem Frame und Konstruktions-Frame grundsätzlich möglich und auch belegt ist (vgl. Unterabschnitt 8.2.1), stellen sie gegenüber der direkten Untersuchung von LE, wie sie die Kollostruktionsanalyse praktiziert, eine inhärente semantische Generalisierung dar, da sie eine semantische Gruppierung der LE nach Frames vorwegnehmen. Wie in Unterabschnitt 5.3.3 gezeigt, stellen lexikalische Frames

52 Gewissermaßen lassen sich die bei FrameNet für einen Frame dokumentierten LE als Verbklasse oder zumindest als Alternative dazu verstehen (vgl. etwa Baker \& Ruppenhofer 2002; Boas 2006). Auch Perek (2015: 118-119) erwägt FrameNet als Grundlage für die Klassifikation von Verben in Verbklassen zu verwenden, entscheidet sich aufgrund der für seine Zwecke zu niedrigen Abdeckung von LE und einer zu grobkörnigen Zuordnung von LE zu Frames jedoch dagegen. 
in einem konstruktionssemantischen Modell die höchste Form einer Generalisierung über LE dar, wobei lexikalische Bedeutungen auf einer niedrigeren Generalisierungsstufe liegen und LE selbst auf der niedrigsten (vgl. Abbildung 5.3 in Unterabschnitt 5.3.3). Den Konstruktions-Frame also direkt anhand der Frequenzen lexikalischer Frames zu rekonstruieren, besitzt den Vorteil dieser inhärenten Generalisierung, sodass eine etwaige semantische Klassifizierung von Kollexemen im Nachgang zu einer Kollostruktionsanalyse, wie sie Gries \& Stefanowitsch (2010) vorschlagen, nicht mehr nötig ist.

Bei dem Stichwort Frequenz ist darauf hinzuweisen, dass eine Methode zur Ermittlung des Konstruktions-Frames über lexikalische Frames, anders als die Kollostruktionsanalyse, auf rohe Frequenzdaten zurückgreifen muss (vgl. dazu kritisch Gries 2012: 497). Für die Verwendung von Assoziationsmaßen oder anderer statistisch elaborierterer Verfahren wäre ein auf lexikalische Frames annotiertes Korpus nötig, das aber zumindest in vergleichbarer Größe zum DWDS-Kernkorpus 21 sowie für das FrameNet-Release 1.7 nicht existiert. ${ }^{53}$ Aus diesem Grund muss für die Untersuchung frequenter lexikalischer Frames als Basis für die Ermittlung des Konstruktions-Frames auf die exhaustive Annotation der Korpusdaten zu der gegebenen Konstruktion zurückgegriffen werden, die - wie in Unterabschnitt 8.4.1 erläutert - schon für die Feststellung des Systems der Frame-Nähen eines potenziellen Konstruktions-Frames nötig ist.

Die Ausgangsfrage bei der konkreten Anwendung dieser Methode lautet nun, welche Art lexikalischer Frames dafür herangezogen werden muss: relatierte lexikalische Frames oder unrelatierte lexikalische Frames? Präziser müsste die Frage eigentlich lauten: Welches Cluster an ermittelten lexikalischen Frames muss herangezogen werden? Ist bereits der in Unterabschnitt 8.4.2 beschriebene Schritt zur Ermittlung solcher Cluster durchgeführt, bietet es sich an, zunächst dasjenige heranzuziehen, das die zu einem potenziellen Konstruktions-Frame relatierten lexikalischen Frames enthält. Dies liegt auf der Hand, da dieses Cluster im Idealfall auch den Konstruktions-Frame selbst beinhaltet, was die Analyse erfolgversprechender werden lässt. Potenziell unrelatierte lexikalische Frames, insbesondere wenn sie keine durch Frame-zu-Frame-Relationen begründete Cluster bilden, bieten sich demgegenüber nicht an, da die Wahrscheinlichkeit, dass sich der Konstruktions-Frame unter ihnen befindet, als deutlich geringer eingestuft werden muss. Zudem liegen ihre Token-Frequenzen, wie die Analysen zur reflexiven Bewegungskonstruktion und zur reflexiven Partikelverbkonstruktion zeigen,

53 Das SALSA-Korpus (Burchardt et al. 2006, 2009; Rehbein et al. 2012), das das Ergebnis früher Bemühungen um ein deutsches FrameNet darstellt, erfüllt diese beiden Kriterien nicht. Eine Kollostruktionsanalyse innerhalb dieses Korpus wäre jedoch freilich möglich. 
deutlich unter denjenigen relatierter lexikalischer Frames (vgl. für die reflexive Bewegungskonstruktion Tabelle 6.3 in Unterabschnitt 6.2.2 und die Tabellen 6.5 bis 6.7 in Unterabschnitt 6.2.3 sowie für die reflexive Bewegungskonstruktion die Tabellen 6.17 und 6.18 bis 6.20 in Unterabschnitt 6.4.2).

Nachdem diese Voraussetzungen geklärt sind, kann die Methode der frequentesten lexikalischen Frames auf die reflexive Bewegungskonstruktion und die reflexive Partikelverbkonstruktion angewendet werden. Sie eignet sich nur für diese beiden der drei untersuchten Konstruktionen, da für die reflexive WegKonstruktion lediglich ein einziger relatierter lexikalischer Frame belegt ist, wie die Untersuchung von deren Produktivität gezeigt hat (vgl. Unterabschnitt 7.5.2, Tabelle 7.20). Obwohl dieser relatierte lexikalische Frame bereits direkt dem Konstruktions-Frame entspricht, kommt die reflexive Weg-Konstruktion eher für andere Methoden zur Ermittlung des Konstruktions-Frames infrage, insbesondere für die Frage, ob ein KEE oder KorE (bzw. Teile davon) als frame-evozierende LE infrage kommen (vgl. Unterabschnitt 8.5.2).

Die Tabellen 8.8 und 8.9 stellen für die reflexive Bewegungskonstruktion und die reflexive Partikelverbkonstruktion noch einmal die jeweils belegten relatierten lexikalischen Frames dar, jeweils absteigend nach deren Frequenz geordnet. Die Daten entsprechen denjenigen in Tabelle 6.2 in Unterabschnitt 6.2.1. Wie sind diese Daten nun im Hinblick auf die Ermittlung des Konstruktions-Frames zu interpretieren? Zwei Schritte erscheinen dafür sinnvoll: (i) die Betrachtung der direkten Frame-zu-Frame-Relationen der lexikalischen Frames auf den oberen Rängen der beiden Tabellen sowie (ii) ausgehend von einem Frame mögliche über- und untergeordnete Frames, die sich ebenfalls in den oberen Rängen der Frames in den Tabellen wiederfinden.

Der Frame Body_movement, der für beide Konstruktionen den frequentesten relatierten lexikalischen Frame darstellt, ist in FrameNet 1.7 über die BenutztRelation mit insgesamt drei weiteren Frames verbunden. Übergeordnet sind die Frames Body_parts und Motion, untergeordnet der Frame Facial_expression. Von diesen Frames findet sich nur Motion als relatierter lexikalischer Frame für beide Konstruktionen wieder. Der Frame Self_motion, der sowohl für die reflexive Bewegungskonstruktion als auch für die reflexive Partikelverbkonstruktion auf dem zweiten Rang liegt, besitzt Relationen zu insgesamt acht weiteren Frames, die sich auf vier verschiedene Arten von Frame-zu-Frame-Relationen verteilen. Belegt sind davon lediglich der in der Vererbungsrelation übergeordnete und zugleich in Siehe_auch-Relation stehende Frame Motion sowie der untergeordnete Frame Fleeing. Der Frame Cause_motion, der für die reflexive Bewegungskonstruktion auf dem vierten Rang und für die reflexive Partikelverbkonstruktion auf dem dritten Rang liegt, weist Relationen zu 17 weiteren Frames auf, die sich auf sechs unterschiedliche Arten von Frame-zu-Frame-Relationen verteilen. Belegt 
Tab. 8.8: Relatierte lexikalische Frames der reflexiven Bewegungskonstruktion in FrameNet 1.7 nach ihrer Token-Frequenz

\begin{tabular}{lrr}
\hline Lexikalische Frames & Konstrukte & Anteil \\
\hline Body_movement & 246 & $34,55 \%$ \\
Self_motion & 159 & $22,33 \%$ \\
Motion & 105 & $14,75 \%$ \\
Cause_motion & 77 & $10,81 \%$ \\
Change_direction & 43 & $6,04 \%$ \\
Bringing & 17 & $2,39 \%$ \\
Fluidic_motion & 17 & $2,39 \%$ \\
Placing & 14 & $1,97 \%$ \\
Motion_directional & 11 & $1,54 \%$ \\
Evading & 7 & $0,98 \%$ \\
Departing & 6 & $0,84 \%$ \\
Fleeing & 5 & $0,70 \%$ \\
Mass_motion & 2 & $0,28 \%$ \\
Making_faces & 1 & $0,14 \%$ \\
Ride_vehicle & 1 & $0,14 \%$ \\
Undressing & 1 & $0,14 \%$ \\
\hline Gesamt & 712 & $100,00 \%$ \\
\hline
\end{tabular}

sind davon die in der Benutzt-Relation untergeordneten Frames Bringing und Excreting, der in der Sub-Frame-Relation untergeordnete Frame Placing, der in der Kausativ-Relation untergeordnete Frame Motion sowie in der Siehe_auchRelation erneut Bringing und Placing.

Die Betrachtung kann nach den ersten vier Rängen beider Tabellen bereits an dieser Stelle abgeschlossen werden. Es zeigt sich eindeutig, dass die belegten relatierten lexikalischen Frames hinsichtlich ihrer Frame-zu-FrameRelationen allesamt auf ein gemeinsames Zentrum fokussiert sind: Motion. So sind Body_movement und Self_motion diesem (in der Benutzt- bzw. der Vererbungsrelation) untergeordnet, während Cause_motion ihm in der KausativRelation übergeordnet ist. Motion liegt hinsichtlich der Frame-zu-Frame-Relationen unter den jeweils vier frequentesten lexikalischen Frames also genau in der Mitte. Prototypentheoretisch betrachtet stellen Body_movement und Self_motion eine untergeordnete (subordinate) Kategorie dar, während Cause_motion eine übergeordnete (superordinate) Kategorie im Sinne von Rosch et al. (1976: 387389) und Rosch (1978: 31) darstellt. Motion, und dies ist das Entscheidende, liegt 
Tab. 8.9: Relatierte lexikalische Frames der reflexiven Partikelverbkonstruktion in FrameNet 1.7 nach ihrer Token-Frequenz

\begin{tabular}{lrr}
\hline Lexikalische Frames & Konstrukte & Anteil \\
\hline Body_movement & 118 & $38,82 \%$ \\
Self_motion & 56 & $18,42 \%$ \\
Cause_motion & 53 & $17,43 \%$ \\
Motion & 24 & $7,89 \%$ \\
Change_direction & 17 & $5,59 \%$ \\
Motion_directional & 15 & $4,93 \%$ \\
Placing & 11 & $3,62 \%$ \\
Bringing & 8 & $2,63 \%$ \\
Excreting & 1 & $0,33 \%$ \\
Operate_vehicle & 1 & $0,33 \%$ \\
\hline Gesamt & 304 & $100,00 \%$ \\
\hline
\end{tabular}

dabei auf der relevanten Basislevel-Kategorie (basic level). ${ }^{54}$ Somit kommt Motion sowohl für die reflexive Bewegungskonstruktion als auch für die reflexive Partikelverbkonstruktion als Konstruktions-Frame infrage. Zu der Schlussfolgerung, dass der mit einer Konstruktion assoziierte Frame oft auf einer Basislevel-Kategorie verortet werden muss, kommen indes auch Perek \& Patten (2019):

The network of frames can also indicate constructional generalisations at intermediate levels of abstraction. In theory, every frame in the network could be taken to correspond to its own construction, with hierarchical relations between subconstructions matching the frame-to-frame relations. However, it is not clear how useful such a myriad of constructions would be in a constructicon database, especially if it is designed for pedagogical purposes. (Perek \& Patten 2019: 376)

Unschwer zu erkennen ist bei den Ergebnissen zur reflexiven Bewegungskonstruktion und reflexiven Partikelverbkonstruktion darüber hinaus, dass Motion keineswegs der frequenteste relatierte lexikalische Frame für beide Konstruktionen ist, da er für die reflexive Bewegungskonstruktion auf dem dritten Rang und für die reflexive Partikelverbkonstruktion gar auf dem vierten Rang liegt. Somit ist nicht zwangsläufig davon auszugehen, dass der Konstruktions-Frame stets dem frequentesten lexikalischen Frame entsprechen muss. Wenn sich, wie im Falle der beiden hier untersuchten Konstruktionen, ein prototypisches Zentrum herausstellt, um das andere durchaus frequentere Frames orientiert sein können,

54 Vgl. auch Ziem (2008: 344-347, 2014b: 296-299) zu Standardwerten, für die BasislevelKategorien eher infrage kommen als übergeordnete oder untergeordnete Kategorien. 
ist es sogar plausibler, dass ein Frame, der auf einer Basislevel-Kategorie liegt, eher als Konstruktions-Frame zu betrachten ist als der frequenteste lexikalische Frame.

Die Ergebnisse zeigen zudem, dass es plausibel erscheint, anders als etwa Lee-Goldman \& Petruck (2018: 32-33) für die way-Konstruktion, gerade nicht Self_motion als Konstruktions-Frame der reflexiven Bewegungskonstruktion und der reflexiven Partikelverbkonstruktion anzusetzen. Abgesehen davon, dass Self_motion durch die Einschränkung, dass es sich bei dessen FE SELF_MOVER um ein „living being which moves under its own power“ (FrameNet 1.7, Self_motion) handeln muss, gegenüber Motion und dem FE THEME semantisch eingeschränkt ist (dazu schon Unterabschnitt 3.3.2), spricht auch die Generalisierung über die frequentesten lexikalischen Frames eindeutig für Motion als Konstruktions-Frame.

\subsubsection{KEE und KorE als frame-evozierende LE}

Eine einfache Methode, den Konstruktions-Frame einer Konstruktion zu bestimmen, ist, diesen auf ein lexikalisch spezifiziertes Strukturelement zurückzuführen, das in der Lage ist, ihn wie jede andere LE (also auch eine solche, die als KtE des KE EREIGNIS infrage kommt) zu evozieren. Zu diesen Strukturelementen zählen in erster Linie KEE und KorE. Einfach ist diese Methode deshalb, weil sich die Evokation des Konstruktions-Frames in diesem Fall deutlich von der Evokation eines lexikalischen Frames unterscheidet (vgl. Unterabschnitt 8.1.1). Während über die KtE eines KE wie EREIGNIS sowohl unrelatierte lexikalische Frames als auch relatierte lexikalische Frames evoziert werden können, die über den Mechanismus der Spreading Activation (Unterabschnitt 8.2.2) zugleich eine Evokation des Konstruktions-Frames bewirken, sind lexikalisch spezifizierte Strukturelemente wie KEE und KorE tendenziell nicht für die Evokation eines lexikalischen Frames verantwortlich und können damit, sofern sie aus frame-evozierenden LE bestehen oder solche enthalten, potenziell der Evokation des Konstruktions-Frames dienen. So weist für die way-Konstruktion bereits Goldberg (1995: 206) darauf hin, dass das Nomen „way is analyzed as a meaningful element“. Berücksichtigt man dies, so wird deutlich, dass die Methode zur Ermittlung des Konstruktions-Frames kaum von den Mechanismen von dessen Evokation zu trennen ist.

An dieser Stelle sei zunächst an die Argumentation von Szcześniak (2014b, 2019a) erinnert, der die formale Abstraktheit einer Konstruktion mit der Abstraktheit ihrer semantischen Eigenschaften korreliert und dafür plädiert, dass, je lexikalisch spezifizierter eine Konstruktion ist, desto konkreter ihre semantischen Eigenschaften ausfallen müssen (dazu schon Unterabschnitt 5.1.2). Szc- 
ześniak schließt daraus, dass jene semantischen Eigenschaften (hier also: die Konstruktions-Frames) besonders in den Fällen der lexikalischen Spezifizierung einer Konstruktion über ebendiese Spezifizierung rekonstruiert werden können:

One corollary of this is that richer meanings found to occur in a syntactic pattern should be traceable to the lexical material embedded in the pattern. That is, if a construction is claimed to convey an open-class-style contentful meaning, it should be possible to point out a lexical item that is part of the construction and is responsible for that meaning. (Szcześniak 2014b: 19)

Wie bereits in den Unterabschnitten 8.3.1 und 8.3.2 festgestellt, findet sich unter den drei untersuchten Konstruktionen lediglich eine Konstruktion, für die die Evokation des Konstruktions-Frames über ein lexikalisch spezifiziertes Strukturelement plausibel ist. Es handelt sich dabei um die reflexive Weg-Konstruktion und ihr KorE. Ihr KEE und ebenso das KEE der reflexiven Bewegungskonstruktion und der reflexiven Partikelverbkonstruktion kommen für die Evokation des Konstruktions-Frames nicht infrage, da ein Reflexivum wie sich kaum als LE des Frames Motion vorstellbar ist (vgl. schon Unterabschnitt 8.3.1). Aus diesem Grund soll in diesem Unterabschnitt die reflexive Weg-Konstruktion mit ihrem KorE im Vordergrund stehen. Sie ist die einzige der drei untersuchten Konstruktionen, für die die Methode der Ermittlung des Konstruktions-Frames über ein lexikalisch spezifiziertes Strukturelement angewendet werden kann.

Die wesentlichen Eigenschaften des KorE der reflexiven Weg-Konstruktion habe ich bereits in den Unterabschnitten 3.3.2 und 8.3.2 dargestellt, weshalb ich sie an dieser Stelle nicht wiederhole. Relevant für die Ermittlung des KonstruktionsFrames ist allerdings derjenige Bestandteil des KorE, der in der Lage ist, einen Frame zu evozieren. Wie bereits in Unterabschnitt 3.3.2 dargestellt, gehört dazu das Nomen Weg auf der einen Seite, wie in dem Konstrukt in (49) zu sehen, und die Nomen Pfad und Trampelpfad auf der anderen Seite, wie in den Konstrukten in (50) und (51) zu sehen.

(49) Was passiert ist, ist passiert“, und er drehte sich um und \{bahnte sich langsam durch die Büsche [KonE seinen Weg] zurück zum Gutshaus\}. (Boie, Kirsten: Skogland, Ort: Hamburg 2005, S. 185)

(50) \{Paul mußte sich [KorE einen Pfad] zum Holzhaus bahnen\}. (Koneffke, Jan: Paul Schatz im Uhrenkasten, Köln: DuMont Buchverlag 2000, S. 77)

(51) $\{[$ KonE Der Trampelpfad], den Jorge sich in Jahren gebahnt hatte\}, war verschwunden, das Wasser hatte ihn genommen. (Düffel, John von: Houwelandt, Köln: DuMont Literatur und Kunst Verlag 2004, S. 293) 
Um aus diesen KorE-Bestandteilen den Konstruktions-Frame zu ermitteln, ist es zunächst aufschlussreich, sich ihre Verteilung über die Konstrukte der Konstruktion sowie die Frames, die sie evozieren, anzusehen. In Tabelle 8.10 ist diese Verteilung für die frame-evozierenden Bestandteile des KorE der reflexiven WegKonstruktion dargestellt. ${ }^{55}$

Tab. 8.10: Verteilung der frame-evozierenden Bestandteile des KorE der reflexiven WegKonstruktion

\begin{tabular}{llrr}
\hline Frame-evozierende LE & Frame & Konstrukte & Anteil \\
\hline Weg & Self_motion & 25 & $92,60 \%$ \\
Pfad & Roadways & 1 & $3,70 \%$ \\
Trampelpfad & Roadways & 1 & $3,70 \%$ \\
\hline Gesamt & & 27 & $100,00 \%$ \\
\hline
\end{tabular}

Die Verteilung zeigt, dass lediglich drei unterschiedliche frame-evozierende LE als KorE-Bestandteile belegt sind und diese insgesamt zwei verschiedene Frames evozieren, nämlich Self_motion und Roadways. Wie ist dieser Befund im Hinblick auf die Ermittlung des Konstruktions-Frames zu deuten? Auf die relevanten Punkte habe ich bereits in Unterabschnitt 8.3.2 hingewiesen, sodass ich sie hier der Übersicht halber noch einmal wiederhole.

- Die beiden unterschiedlichen Frames könnten auf eine konstruktionelle Polysemie hindeuten, die durch unterschiedliche Konstruktions-Frames bedingt wäre (Unterabschnitt 5.2.1). Dies ist allerdings wenig plausibel, da die semantischen Unterschiede zwischen diesen frame-evozierenden LE gering sind.

- Die semantischen Unterschiede zwischen diesen frame-evozierenden LE sind deshalb gering, weil beide Frames, die sie evozieren, Self_motion und Roadways, in Frame-Nähe zu Motion stehen.

- Die Frame-Nähe dieser beiden Frames zu Motion, die in beiden Fällen +1 beträgt, einmal für die Vererbungsrelation (Self_motion) und einmal für die Benutzt-Relation (Roadways), spricht dafür, diese beiden Frames zu einem allgemeinen Frame zu generalisieren, der dann als Konstruktions-Frame anzunehmen ist (vgl. auch Perek \& Patten 2019: 376). Dieser Frame ist Motion.

55 Wie bereits in Unterabschnitt 8.3.2 erwähnt, ist das Nomen Trampelpfad in FrameNet 1.7 nicht als eigene LE des Frames Roadways verzeichnet. Stattdessen gehe ich davon aus, dass das Determinativkompositum Trampelpfad ebenso wie das Simplizium Pfad ebendiesen Frame evoziert. 
Mit diesen Beobachtungen ist die Methode zur Ermittlung des KonstruktionsFrames für die reflexive Weg-Konstruktion nahezu vollständig. Die einzige Besonderheit besteht nun darin, dass nicht die Frames, die direkt von den frameevozierenden KorE-Bestandteilen evoziert werden (Self_motion und Roadways), als Konstruktions-Frames angesetzt werden, sondern ein Frame, zu dem diese beiden Frames in Frame-Nähe stehen. Dieser Schritt der Generalisierung mag nicht für jede Konstruktion nötig sein, die über ein lexikalisch fixiertes Strukturelement verfügt, das einen Frame evoziert, für die reflexive Weg-Konstruktion jedoch erscheint sie plausibel. Hinzu kommt, dass die Höhe dieser Generalisierung zumindest für die reflexive Weg-Konstruktion in Grenzen bleiben kann, da sowohl Self_motion als auch Roadways in einer Frame-Nähe von lediglich +1 (und nicht +2 oder niedriger) zum letztendlichen Konstruktions-Frame Motion stehen. Diese Zusammenhänge sind in Abbildung 8.8 zusammenfassend dargestellt.

Frame-Nähe

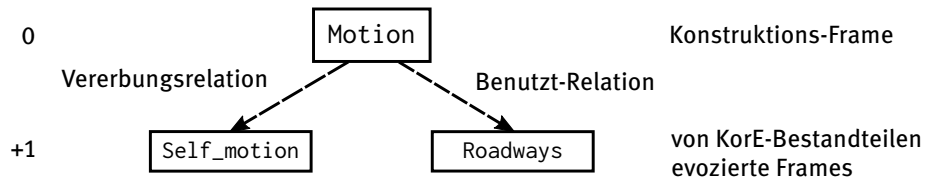

Abb. 8.8: Frame-Nähe zwischen den Frames der frame-evozierenden Bestandteile des KorE und dem Konstruktions-Frame der reflexiven Weg-Konstruktion

Die reflexive Weg-Konstruktion ist unter den drei untersuchten Konstruktionen jedoch nicht nur die am wenigsten formal abstrakte (vgl. Unterabschnitt 5.1.2) weshalb sie für die soeben vorgestellte Methode zur Ermittlung des KonstruktionsFrames prädestiniert ist -, sie ist ebenso die mit Abstand am wenigsten produktivste der drei untersuchten Konstruktionen (vgl. Unterabschnitt 7.5.2). Wenn sie, wie im Eingang zu diesem Unterabschnitt festgestellt, die einzige der drei untersuchten Konstruktionen ist, deren Konstruktions-Frame sich durch frameevozierende LE als Bestandteile von KEE oder KorE ermitteln lässt, müssen für die beiden anderen Konstruktionen, die reflexive Bewegungskonstruktion und die reflexive Partikelverbkonstruktion, andere Methoden zur Ermittlung des Konstruktions-Frames herangezogen werden. Eine davon habe ich bereits mit der Ermittlung der frequentesten relatierten lexikalischen Frames in Unterabschnit 8.5.1 vorgestellt. Auf eine zweite Methode möchte ich im folgenden Unterabschnitt 8.5.3 eingehen. 


\subsubsection{Paraphrasieren}

Die letzte der drei Methoden zur Ermittlung des Konstruktions-Frames bedient sich eines in der Linguistik wohletablierten Verfahrens: des Paraphrasierens. Paraphrasen sind bereits außerhalb einer dezidierten Beschäftigung mit semantischen Fragestellungen, aber z.B. auch in der traditionellen Lexikographie wohl das mit Abstand beliebteste linguistische Mittel, um semantische Eigenschaften zu beschreiben (vgl. Busse 2015b: 94; Sikos \& Padó 2018: 38-39). Auch in der Konstruktionsgrammatik werden Paraphrasen bisweilen zur semantischen Beschreibung von Konstruktionen herangezogen: Goldberg (1995: 3-4) nutzt sie zur initialen Charakterisierung der von ihr untersuchten ArgumentstrukturKonstruktionen.

Paraphrasen können jedoch auch für eine semantische Theorie wie die FrameSemantik gewinnbringend sein, besonders dann, wenn semantische Eigenschaften offengelegt werden sollen, die nicht auf lexikalischer Ebene zu beobachten sind. Aus konstruktionssemantischer Sicht heißt dies: Paraphrasen können insbesondere dann zur Ermittlung des Konstruktions-Frames herangezogen werden, wenn der Konstruktions-Frame nicht durch ein einzelnes lexikalisches Element, sei es das KtE eines KE (Unterabschnitt 8.1.2), ein KEE (Unterabschnitt 8.3.1) oder ein KorE (Unterabschnitt 8.3.2), evoziert wird. Somit ist, wie in Unterabschnitt 8.4.1 erwähnt, die Methode des Paraphrasierens insbesondere für Konstrukte mit unrelatierten lexikalischen Frames relevant, in denen die Evokation des Konstruktions-Frames über eine syntagmatische Kombination von Strukturelementen (Unterabschnitt 8.3.3) stattfindet und eben nicht auf eine einzelne LE rückführbar ist, die sich in FrameNet nachschlagen ließe. Deshalb kann die Methode des Paraphrasierens besonders auf die reflexive Bewegungskonstruktion und die reflexive Partikelverbkonstruktion angewendet werden, da ihre Konstrukte mit unrelatierten lexikalischen Frames jene Evokation des Konstruktions-Frames aufweisen.

Welchen konkreten semantischen Nutzen haben Paraphrasen? Im Rahmen satzsemantischer Überlegungen nutzt von Polenz (2008: 65) das Mittel der „maximal explizite[n] normalsprachliche[n] Paraphrase“, um Prädikationsstrukturen, die aus Prädikaten und deren Referenzstellen bestehen, zu analysieren. Eine solche Paraphrase kann gleichermaßen bei „Ziel und Aufgabe der satzsemantischen Analyse [...], sämtliche Prädikate und sämtliche Bezugsstellen, die ein Sprecher in einem Satz ausdrückt - ob explizit oder implizit - offenzulegen“ (Busse 2015b: 136) eine große Hilfe sein. Bei Sätzen, die eine Vielzahl von Prädikationen in komprimierter Form ausdrücken, sieht von Polenz (2008: 94) Paraphrasen als geeignet an, „, $[\mathrm{u}] \mathrm{m}$ auch alle Aussagen-Einbettungen [...] mitsamt ihren Referenzstellen [...] herauszubekommen“. Mit anderen Worten: „Paraphrasen sind bei Sätzen 
dasselbe wie Synonyme bei Wörtern“ (von Polenz 2008: 79).56 Frame-semantisch reformuliert bedeutet dies Folgendes: Paraphrasen sind eine effektive Methode, um Frames sichtbar zu machen, die nicht von einzelnen LE in dem Konstrukt, das Gegenstand der Paraphrase ist, evoziert werden. Das Prädikat, das die Paraphrase offenlegen soll, entspricht dabei also der LE, die den betreffenden Frame evoziert. Eine plakative Analyse des Artikels 5,3 des Grundgesetzes führt bei von Polenz (2008: 242-246) zu einem Ergebnis, das Busse (2015b: 139) wie folgt zusammenfasst: „Statt der syntaktisch ausgedrückten $z$ wei ergibt die satzsemantische Analyse [...] neun Prädikationen, statt der ausgedrückten sechs ergeben sich mindestens zwölf Bezugsstellen. “ In satzsemantischer Methodik sind Paraphrasen somit „Ausgangsmaterial der Beschreibung der Prädikationsstruktur“ (Busse 2015b: 135). Auf frame-semantische Begriffe gebracht heißt dies nichts anderes als dass Paraphasen dabei helfen können, die Evokation (also die LE, die ihn evozieren) einerseits und die Struktur eines Frames (also seine instanziierten

56 Auf Grundlage der Benutzt-Relation von FrameNet entwickeln Sikos \& Padó (2018) ein elaborierteres Modell für konzeptbasierte Paraphrasen (concept-based paraphrases), die gerade nicht auf einer strikten Synonymie-Relation zwischen Wörtern oder Sätzen beruhen, sondern deren Verbindung zum Ausgangssatz durch die Benutzt-Relation hergestellt wird, etwa dadurch, dass die Prädikate im Ausgangssatz und in der Paraphrase durch ebendiese Frame-zu-Frame-Relation verbunden sind. Auf den ersten Blick erscheint dieses Verfahren objektivierbarer als von Polenz' introspektive Herangehensweise, allerdings ist es gerade durch die Festlegung auf die Benutzt-Relation eingeschränkt. Wie die Analysen in Unterabschnitt 6.2.1 gezeigt haben, können Konstrukt-Frames der reflexiven Bewegungskonstruktion (und ebenso der reflexiven Partikelverbkonstruktion) durchaus durch einen lexikalischen Frame, der in einer Benutzt-Relation zum Konstruktions-Frame steht, konstituiert werden. Diese Motivierung betrifft allerdings jenen Teil der Konstrukte, deren Konstrukt-Frames aus relatierten lexikalischen Frames bestehen und somit nur jene Fälle, in denen der Konstruktions-Frame durch einen Spreading-Activation-Prozess (Unterabschnitt 8.2.2) lediglich ,mitevoziert‘ wird. Anteile des Konstruktions-Frames an der Konstitution eines Konstrukt-Frames bei einem unrelatierten lexikalischen Frame - worum es in diesem Unterabschnitt also primär geht - können damit nicht erfasst werden. Deshalb eignet sich die Benutzt-Relation nicht, um systematisch Paraphrasen von Konstrukten zu konstruieren: Da die Paraphrase eine LE enthalten soll, die direkt den potenziellen Konstruktions-Frame evoziert, könnten nur Konstrukte paraphrasiert werden, deren lexikalische Frames in einer BenutztRelation zum jenem Konstruktions-Frame stehen. Diese aber machen, wie erwähnt, nur einen kleinen Teil aller Konstrukte aus. Auch der Einbezug anderer Frame-zu-Frame-Relation, wie ihn etwa Hasegawa et al. (2011: 110-115) vorschlagen, könnte letztendlich nur Paraphrasen für diejenigen Konstrukte ergeben, die vollständig einfach durch einen relatierten lexikalischen Frame motiviert sind (im Sinne der Analyse in Unterabschnitt 6.2.1). Dies ist eine weitere Begründung dafür, warum die Methode des Paraphrasierens gerade für Konstrukte mit unrelatierten lexikalischen Frames angewendet werden soll, wobei jene lexikalischen Frames eben in keiner Framezu-Frame-Relation zum Konstruktions-Frame stehen. 
FE) andererseits offenzulegen. ${ }^{57}$ Somit bieten sich Paraphrasen geradezu an, um einen nur durch die syntagmatische Kombination von Strukturelementen evozierten Konstruktions-Frame zu ermitteln. Paraphrasen können im Rahmen einer konstruktionssemantischen Analyse einerseits dafür eingesetzt werden, den Konstruktions-Frames unter Rückschlüssen auf LE, die für seine Evokation infrage kommen, zu ermitteln, und andererseits, mögliche instanziierte FE offenzulegen, die einzelne KtE eines Konstrukts (einfach oder doppelt) motiveren. Da es im vorliegenden Zusammenhang in erster Linie um die Ermittlung des Frames an sich geht, klammere ich Letzteres aus.

Anhand dieser Überlegungen wird deutlich, auf welche konkreten Arbeitsschritte die Methode des Paraphrasierens zusteuern muss. Anders als es die Analysen von von Polenz und Busse suggerieren, ist nämlich keineswegs eine „maximal explizite normalsprachliche Paraphrase“ (von Polenz 2008: 65) vonnöten, um einen Konstruktions-Frame zu ermitteln, gerade weil es in erster Linie darum geht, eine LE, die in $\mathrm{zu}$ evozieren in der Lage ist, $\mathrm{zu}$ rekonstruieren. Somit sind bereits recht einfache Paraphrasen sehr effektiv, um einen Konstruktions-Frames auf der Basis von Konstrukten mit unrelatierten lexikalischen Frames offenzulegen. Der wichtigste Schritt beim Paraphrasieren eines Konstrukts mit einem unrelatierten lexikalischen Frame ist es also, in die Paraphrase dieses Konstrukts eine LE einzufügen, die den potenziellen Konstruktions-Frame evoziert. Welche LE und somit welche Frames kommen dafür infrage? An dieser Stelle kommt die methodologische Vorarbeit der Ermittlung des Systems der Frame-Nähe des potenziellen Konstruktions-Frames (Unterabschnitt 8.4.2) ins Spiel. Die Methode des Paraphrasierens adressiert in erster Linie Konstrukte mit unrelatierten lexikalischen Frames. Der Grund dafür liegt auf der Hand: Bei der Untersuchung der relatierten lexikalischen Frames ist zu erwarten, dass der Konstruktions-Frame unter ihnen ist (vgl. Unterabschnitt 8.5.1), weshalb Konstrukte mit ihnen nicht der Methode des Paraphrasierens unterzogen werden müssen. Gleichzeitig aber kann diese Methode auf das bereits ermittelte System potenzieller Frame-Nähen eines Konstruktions-Frames zurückgreifen. Da die Menge der darin befindlichen relatierten lexikalischen Frames, unter denen sich der potenzielle KonstruktionsFrame befindet, eingeschränkt ist, bietet es sich an, zuerst die LE dieser Frames in

57 Allerdings wendet Busse (2015b: 135) zurecht ein: „[E]ine Paraphrase setzt aber schon eine Interpretation des Satzes voraus. Satzsemantik, wie von Polenz sie vorschlägt, kann also nur auf der Basis bereits verstandener Satzinhalte operieren.“ Diese Einschränkung resultiert darin, „dass auch die Satzsemantik (wie schon die Wortsemantik) keine Methode der Bedeutungserschließung bereitstellt; sie stellt allerdings [...] eine exzellente Methode der Verdeutlichung von Satzbedeutungen, [...], dar“ (Busse 2015b: 135). Zum Begriff der Bedeutungserschließung in Abgrenzung zu einer Bedeutungsbeschreibung vgl. auch Busse (2015a: 54). 
die Paraphrasen der Konstrukte mit unrelatierten lexikalischen Frames einzufügen und zu prüfen, ob eine plausible Interpretation entsteht, die den entsprechenden relatierten lexikalischen Frame als Konstruktions-Frame identifiziert. Um die Auswahl der relatierten lexikalischen Frames zu vereinfachen, können zunächst diejenigen getestet werden, die bei der Analyse der frequentesten lexikalischen Frames (Unterabschnitt 8.5.1) die oberen Ränge belegen. Ich beschränke die Auswahl auf die jeweils vier frequentesten Frames, zumal nach ihnen bereits die dortige Suche nach einem Konstruktions-Frame abgeschlossen werden konnte.

Ich möchte die Methode im Folgenden an jeweils einem Konstrukt der reflexiven Bewegungskonstruktion und der reflexiven Partikelverbkonstruktion demonstrieren. Als Ausgangskonstrukt der reflexiven Bewegungskonstruktion soll jenes in (52) mit dem lexikalischen Frame Scouring dienen. Dabei genügt es, lediglich das einschlägige Konstrukt, das hier durch geschweifte Klammern markiert ist, zu paraphrasieren.

(52) Ich arbeitete gut und konzentriert; ich hatte Kräfte für zehn - und doch schlugen mir die Knie aneinander, als ich in die Allee einbog, und \{meine Hände gruben sich in die Rocktaschen\}, bis sie beinahe rissen. (Schmitter, Elke: Frau Sartoris, Berlin: BvT 2000[2002], S. 29)

Zur Erinnerung: Für die reflexive Bewegungskonstruktion wurden in Unterabschnitt 8.5.1 die relatierten lexikalischen Frames Body_movement, Self_motion, Motion und Cause_motion in dieser Reihenfolge auf den ersten vier Rängen der frequentesten aller belegten relatierten lexikalischen Frames ermittelt (vgl. Tabelle 8.8 in Unterabschnitt 8.5.1). Diese Frames verfügen in FrameNet 1.7 über teils enorm zahlreiche LE mit sehr unterschiedlichen lexikalischen Bedeutungen. Um nicht alle dieser LE exhaustiv in entsprechenden Paraphrasen testen zu müssen, genügt es, eine Auswahl von LE zu treffen, deren lexikalische Bedeutungen einer neutralen Lesart oder - wenn keine neutrale Lesart belegt ist - einer ,manner Lesart zuzuordnen sind (vgl. dazu Unterabschnitt 5.2.3). Um die Auswahl noch weiter einzugrenzen, bietet es sich an, auf diejenigen LE zurückzugreifen, die für die entsprechenden relatierten lexikalischen Frames in der Datenauswahl für die Konstruktion belegt sind. Diese sind für die Analyse der konstruktionellen Polysemie, die, wie in Unterabschnitt 5.2.2 argumentiert, durch variierende lexikalische Frames und lexikalische Bedeutungen zustande kommt, bereits auf die unterschiedlichen Lesarten der drei untersuchten Konstruktionen hin annotiert, sodass diejenigen LE mit einer neutralen oder auf einen ,manner-Aspekt spezifizierten lexikalischen Bedeutung einfach identifiziert werden können. Ich gehe die vier relatierten lexikalischen Frames und ihre LE nun im Einzelnen durch. 
Für den frequentesten Frame Body_movement sind die LE beugen (flex.v), biegen (bend.v), hängen (hang.v), heben (lift.v) und schließen (close.v) belegt (vgl. Tabelle 5.2 in Unterabschnitt 5.2.3). Das Konstrukt in (52) kann nun entsprechend paraphrasiert werden, indem diese LE in die Paraphrase eingefügt werden, wobei die ursprüngliche LE, die den unrelatierten lexikalischen Frame (hier: Scouring) evoziert, in einen Nebensatz mit indem verschoben werden kann, da das ursprüngliche Konstrukt in (52) eine ,means'-Lesart besitzt. ${ }^{58}$ Das Ergebnis sind die Paraphrasen in (53), wobei die nunmehr relatierten lexikalischen Frames, die die eingefügten LE evozieren, zur Übersicht annotiert sind.

(53) a. ?Meine Hände [Body_movement beugten] sich in die Rocktaschen, indem sie gruben.

b. ??Meine Hände [Body_movement bogen] sich in die Rocktaschen, indem sie gruben.

c. ??Meine Hände [Body_movement hingen] sich in die Rocktaschen, indem sie gruben.

d. ??Meine Hände [Body_movement hoben] sich in die Rocktaschen, indem sie gruben.

e. ??Meine Hände [Body_movement Schlossen] sich in die Rocktaschen, indem sie gruben.

Die traditionell auch in Grammatikalitätsurteilen $\mathrm{zu}$ findenden Kennzeichnungen aus Fragezeichen sollen die Plausibilität der Interpretation der einzelnen Paraphrasen im Hinblick auf die Konstruktion andeuten. Je mehr Fragezeichen dabei verwendet werden, desto unplausibler erscheint die Paraphrase. Wie deutlich zu erkennen ist, ist die LE beugen in dieser Hinsicht noch am wenigsten problematisch, ergibt aber keine gänzlich plausible Interpretation. Der Frame Body_movement erscheint also als Konstruktions-Frame eher ungeeignet.

Der auf dem zweiten Rang liegende Frame Self_motion ist, wie in Tabelle 5.2 in Unterabschnitt 5.2.3 zu sehen, für die neutrale Lesart nicht belegt, stattdessen sind für ihn zahlreiche LE mit ,manner'-Lesart belegt, auf deren Basis nun entsprechende Paraphrasen zusammengestellt werden können. Dies betrifft die LE drängen (press.v), drücken (press.v), hangeln (clamber.v), kriechen (creep.v),

58 Vgl. dazu die entsprechenden Paraphrasen von Oya (1999: 356-357). Zu beachten ist hier auch die Klassifikation von indem durch Duden (2016: 1063) als ,modal', was Smirnova (2018: 21) zufolge jedoch als ,manner'-Lesart zu verstehen ist. An anderer Stelle klassifiziert Duden (2016: 640) indem als Mitglied der Klasse der „[m]odal-instrumentale[n] Subjunktionen“ - wobei Letzteres zur Übersetzung von ,means“ mit instrumental durch Smirnova (2018: 21) passt. Um eine solche Vermischung semantischer Beschreibungen zu vermeiden, gehe ich davon aus, dass indem eine ,instrumentale‘ und damit eine ,means'-Lesart besitzt. 
pirschen (stalk.v), pressen (press.v), reißen (rip.v), robben (crawl.v), schleichen (sneak.v), stehlen (steal.v), stemmen (press.v), stürzen (pounce.v) und verkriechen (creep.v) belegt. Die entsprechenden Paraphrasen finden sich in (54).

a. ??Meine Hände [self_motion drängten] sich in die Rocktaschen, indem sie gruben.

b. ??Meine Hände [self_motion drückten] sich in die Rocktaschen, indem sie gruben.

c. ??Meine Hände [self_motion hangelten] sich in die Rocktaschen, indem sie gruben.

d. ??Meine Hände [self_motion krochen] sich in die Rocktaschen, indem sie gruben.

e. ??Meine Hände [self_motion pirschten] sich in die Rocktaschen, indem sie gruben.

f. ?Meine Hände [self_motion pressten] sich in die Rocktaschen, indem sie gruben.

g. ??Meine Hände [self_motion rissen] sich in die Rocktaschen, indem sie gruben.

h. ??Meine Hände [self_motion robbten] sich in die Rocktaschen, indem sie gruben.

i. ?Meine Hände [self_motion schlichen] sich in die Rocktaschen, indem sie gruben.

j. ??Meine Hände [self_motion stahlen] sich in die Rocktaschen, indem sie gruben.

k. ?Meine Hände [self_motion stemmten] sich in die Rocktaschen, indem sie gruben.

1. ??Meine Hände [self_motion stürzten] sich in die Rocktaschen, indem sie gruben.

m.??Meine Hände [self_motion verkrochen] sich in die Rocktaschen, indem sie gruben.

Der Frame Self_motion erscheint insgesamt weniger problematisch als Body movement, da gleich mehrere Paraphrasen - diejenigen mit den LE pressen, schleichen und stemmen - nicht gänzlich unplausibel erscheinen. Alle anderen Paraphrasen erscheinen deutlich problematischer, allerdings gibt das gesamte Bild noch keinen Anlass dazu, Self_motion als Konstruktions-Frame festzulegen.

Als nächstes ist Motion an der Reihe, der auf dem dritten Rang der frequentesten lexikalischen Frames liegt. Für ihn sind in neutraler Lesart die LE begeben (go.v), bewegen, rücken, rühren und verschieben (alle move.v) belegt. Die entsprechenden Paraphrasen sind in (55) zu sehen. 
(55) a. Meine Hände [Motion begaben] sich in die Rocktaschen, indem sie gruben.

b. Meine Hände [Motion bewegten] sich in die Rocktaschen, indem sie gruben.

c. ?Meine Hände [Motion rückten] sich in die Rocktaschen, indem sie gruben.

d. ??Meine Hände [Motion rührten] sich in die Rocktaschen, indem sie gruben.

e. ?Meine Hände [Motion verschoben] sich in die Rocktaschen, indem sie gruben.

Deutlich zu erkennen ist, dass insbesondere die Paraphrasen mit den LE begeben und bewegen unproblematisch sind, was Motion bereits an dieser Stelle deutlich stärker als Konstruktions-Frame als Body_movement und Self_motion qualifiziert. Bevor ein abschließendes Urteil gefällt werden kann, fehlen allerdings noch die Paraphrasen mit dem viertfrequentesten relatierten lexikalischen Frame, nämlich Cause_motion. Seine für die neutrale Lesart belegten LE sind heben (lift.v), schieben (push.v), stoßen (thrust.v) und ziehen (pull.v). Die Paraphrasen finden sich in (56).

(56) a. ??Meine Hände [Cause_motion hoben] sich in die Rocktaschen, indem sie gruben.

b. ?Meine Hände [Cause_motion Schoben] sich in die Rocktaschen, indem sie gruben.

c. ?Meine Hände [Cause_motion stießen] sich in die Rocktaschen, indem sie gruben.

d. ??Meine Hände [Cause_motion zogen] sich in die Rocktaschen, indem sie gruben.

Cause_motion ist auf der Grundlage dieser Paraphrasen ähnlich qualifiziert wie Self_motion: Keine der Paraphrasen erscheint gänzlich unproblematisch. Es wird also deutlich, dass unter diesen vier relatierten lexikalischen Frames Motion am ehesten als Konstruktions-Frame infrage kommt, da nur er Paraphrasen erlaubt, die gänzlich unproblematisch sind und somit keine Auszeichnung durch ein Fragezeichen bekommen müssen. Diese Ergebnisse decken sich mit denjenigen der Analyse der frequentesten relatieren lexikalischen Frames in Unterabschnitt 8.5.1. Mehr noch: Die Methode des Paraphrasierens kann gleichzeitig dazu genutzt werden, die Ergebnisse der Analyse dieser anderen Methode abzusichern. Entsteht bei demjenigen Frame, der dort als Konstruktions-Frame ermittelt wurde, eine plausible Interpretation mindestens einer Paraphrase im Sinne der 
gegebenen Konstruktion, wie dies bei Motion der Fall ist, deutet dies auf eine plausible Ermittlung des Konstruktions-Frames schon über diese Methode hin.

Zum Abschluss möchte ich die Methode des Paraphrasierens auf die reflexive Partikelverbkonstruktion anwenden. Die relatierten lexikalischen Frames, die in Unterabschnitt 8.5.1 für sie ermittelt wurden, sind dieselben wie für die reflexive Bewegungskonstruktion, allerdings in anderer Reihenfolge: Body_movement, Self_motion, Cause_motion und Motion (vgl. Tabelle 8.9 in Unterabschnitt 8.5.1). Zudem unterscheiden sich die für diese Frames mit der reflexiven Partikelverbkonstruktion belegten LE teilweise von denjenigen der reflexiven Bewegungskonstruktion, weshalb die oben ermittelten LE nur teils auch auf die reflexive Partikelverbkonstruktion angewendet werden können. Als Ausgangskonstrukt für die folgenden Paraphrasen sei das Konstrukt in (57) mit dem lexikalischen Frame Seeking herangezogen.

(57) \{Die Frau tastet sich an der Wand entlang zu der Alten hinüber $\}$ und hockt sich schwerfällig neben sie hin. (Venske, Regula: Marthes Vision, Frankfurt am Main: Eichborn Verlag 2006, S. 189)

Wie für die reflexive Bewegungskonstruktion ist auch für die reflexive Partikelverbkonstruktion Body_movement der frequenteste relatierte lexikalische Frame. Für ihn sind in neutraler Lesart die Basisverb-LE beugen (flex.v), biegen, bücken (beide bend.v) und heben (lift.v) belegt (vgl. Tabelle 5.3 in Unterabschnitt 5.2.3). Die entsprechenden Paraphrasen sind in (58) zu sehen, wobei die ursprüngliche LE, die den unrelatierten lexikalischen Frame Seeking evoziert, in ein Modaladverb überführt wird, weil das Konstrukt - anders als dasjenige der reflexiven Bewegungskonstruktion in (52) - eine ,manner'-Lesart besitzt. ${ }^{59}$

a. ?Die Frau [Body_movement beugt] sich tastend an der Wand entlang zu der Alten hinüber.

b. ??Die Frau [Body_movement biegt] sich tastend an der Wand entlang zu der Alten hinüber.

c. ?Die Frau [Body_movement bückt] sich tastend an der Wand entlang zu der Alten hinüber.

d. ??Die Frau [Body_movement hebt] sich tastend an der Wand entlang zu der Alten hinüber.

59 Vgl. dazu Duden (2016: 588): „Modaladverbien [...] geben sowohl die Qualität (Art und Weise) als auch die Quantität (Menge, Ausmaß) an. In der Regel lassen sie sich mit wie? erfragen.“ (Hervorhebung im Original in Fettdruck, A.W.). 
Zwei dieser Paraphrasen, diejenigen mit den LE beugen und bücken, erscheinen weniger unproblematisch als die anderen Paraphrasen, erzeugen jedoch eine sehr spezifische Lesart, die über den Kontext des Konstrukts kaum abgedeckt zu sein scheint. Body_movement scheint für die reflexive Partikelverbkonstruktion deshalb ebenso wie für die reflexive Bewegungskonstruktion nicht als Konstruktions-Frame geeignet.

Der nächstfrequenteste Frame ist Self_motion. Belegt sind für ihn, wie in Tabelle 5.3 in Unterabschnitt 5.2.3 zu sehen, nur LE in ,manner'-Lesart, weshalb diese für die Paraphrasen herangezogen werden. Es handelt sich um drängen (press.v), drücken (press.v), fuhrwerken (bustle.v), pirschen (stalk.v), ranken (climb.v), schaukeln (swing.v), schleichen (sneak.v), schwimmen (swim.v), stemmen (press.v) und stürzen (pounce.v). Die entsprechenden Paraphrasen finden sich in (59).

(59) a. ??Die Frau [self_motion drängt] sich tastend an der Wand entlang zu der Alten hinüber.

b. ??Die Frau [self_motion drückt] sich tastend an der Wand entlang zu der Alten hinüber.

c. ??Die Frau [self_motion fuhrwerkt] sich tastend an der Wand entlang zu der Alten hinüber.

d. ?Die Frau [self_motion pirscht] sich tastend an der Wand entlang zu der Alten hinüber.

e. ??Die Frau [self_motion rankt] sich tastend an der Wand entlang zu der Alten hinüber.

f. ??Die Frau [self_motion schaukelt] sich tastend an der Wand entlang zu der Alten hinüber.

g. ?Die Frau [self_motion schleicht] sich tastend an der Wand entlang zu der Alten hinüber.

h. ??Die Frau [self_motion schwimmt] sich tastend an der Wand entlang zu der Alten hinüber.

i. ??Die Frau [self_motion stemmt] sich tastend an der Wand entlang zu der Alten hinüber.

j. ??Die Frau [self_motion stürzt] sich tastend an der Wand entlang zu der Alten hinüber.

Es scheint offensichtlich, dass alle Paraphrasen bis auf diejenigen mit den LE pirschen und schleichen die bisher unplausibelsten Interpretationen ergeben.

Auf dem dritten Rang liegt schließlich Cause_motion. Für ihn sind in neutraler Lesart die (Basisverb-)LE abstoßen (push.v), heben (lift.v), schieben (push.v) und ziehen (pull.v) belegt, die in den Paraphrasen in (60) zu sehen sind. 
a. ??Die Frau [Cause_motion stößt] sich tastend an der Wand entlang zu der Alten hinüber ab.

b. ??Die Frau [Cause_motion hebt] sich tastend an der Wand entlang zu der Alten hinüber.

c. ?Die Frau [Cause_motion schiebt] sich tastend an der Wand entlang zu der Alten hinüber.

d. ??Die Frau [Cause_motion zieht] sich tastend an der Wand entlang zu der Alten hinüber.

Bei Cause_motion erscheint nur eine Paraphrase wenig problematisch, insgesamt kommt er dem Konstruktions-Frame näher als Body_movement und besonders Self_motion.

Der letzte Frame ist der, der bereits für die reflexive Bewegungskonstruktion als Konstruktions-Frame ermittelt werden konnte: Motion. Belegt ist für ihn in neutraler Lesart einzig die LE bewegen (move.v), deren Paraphrase in (61) zu sehen ist.

(61) Die Frau [Motion bewegt] sich tastend an der Wand entlang zu der Alten hinüber.

Wie schon für die reflexive Bewegungskonstruktion scheint Motion auch für die reflexive Partikelverbkonstruktion über die Methode des Paraphrasierens als Konstruktions-Frame zu identifizieren zu sein. Die Paraphrase mit der LE bewegen ist ein eindeutiger Beleg dafür, sie erscheint noch unproblematischer als die Paraphrasen für den Frame Cause_motion. Die Ergebnisse dieser Methode decken sich für die reflexive Partikelverbkonstruktion somit ebenfalls mit denjenigen der Methode der frequentesten relatierten lexikalischen Frames (Unterabschnitt 8.5.1).

Dass die Methode des Paraphrasierens zur Ermittlung des KonstruktionsFrames zu plausiblen Ergebnissen führt, widerlegt für die drei untersuchten Konstruktionen die Position von Szcześniak (2014b: 154-155), der eine fehlende Paraphrasierbarkeit für die way-Konstruktion (bzw. einige ihrer Konstrukte) annimmt (dazu schon Unterabschnitt 4.3.3). Noch deutlicher zeigt sich aber, dass die drei diskutierten Methoden komplementär zueinander stehen. Die Ermittlung des Konstruktions-Frames über die frequentesten relatierten lexikalischen Frames (Unterabschnitt 8.5.1) sollte jedenfalls zuerst vorgenommen werden, bevor die Methode des Paraphrasierens angewendet wird. Sie ist auch deswegen lediglich als Ultima Ratio zu betrachten, weil sie nicht nur auf introspektiven Plausibilitätsurteilen beruht, sondern gänzlich losgelöst von Frames operiert und somit auch keine semantischen Parameter wie die Frame-Nähe eines lexikalischen Frames berücksichtigt. Dies sind ebenso die Gründe dafür, warum diese Methode in diesem Kapitel als letztes betrachtet wird. Für den Fall aber, dass sowohl die 
Methode der frequentesten relatierten lexikalischen Frames als auch diejenige des Paraphrasierens zu demselben plausiblen Ergebnis über den KonstruktionsFrame einer gegebenen Konstruktion kommen, kann davon ausgegangen werden, dass sie nicht nur einzeln funktionieren, sondern gemeinsam die Grundlage für eine methodisch gesicherte Annahme eines Konstruktions-Frames bilden. 